
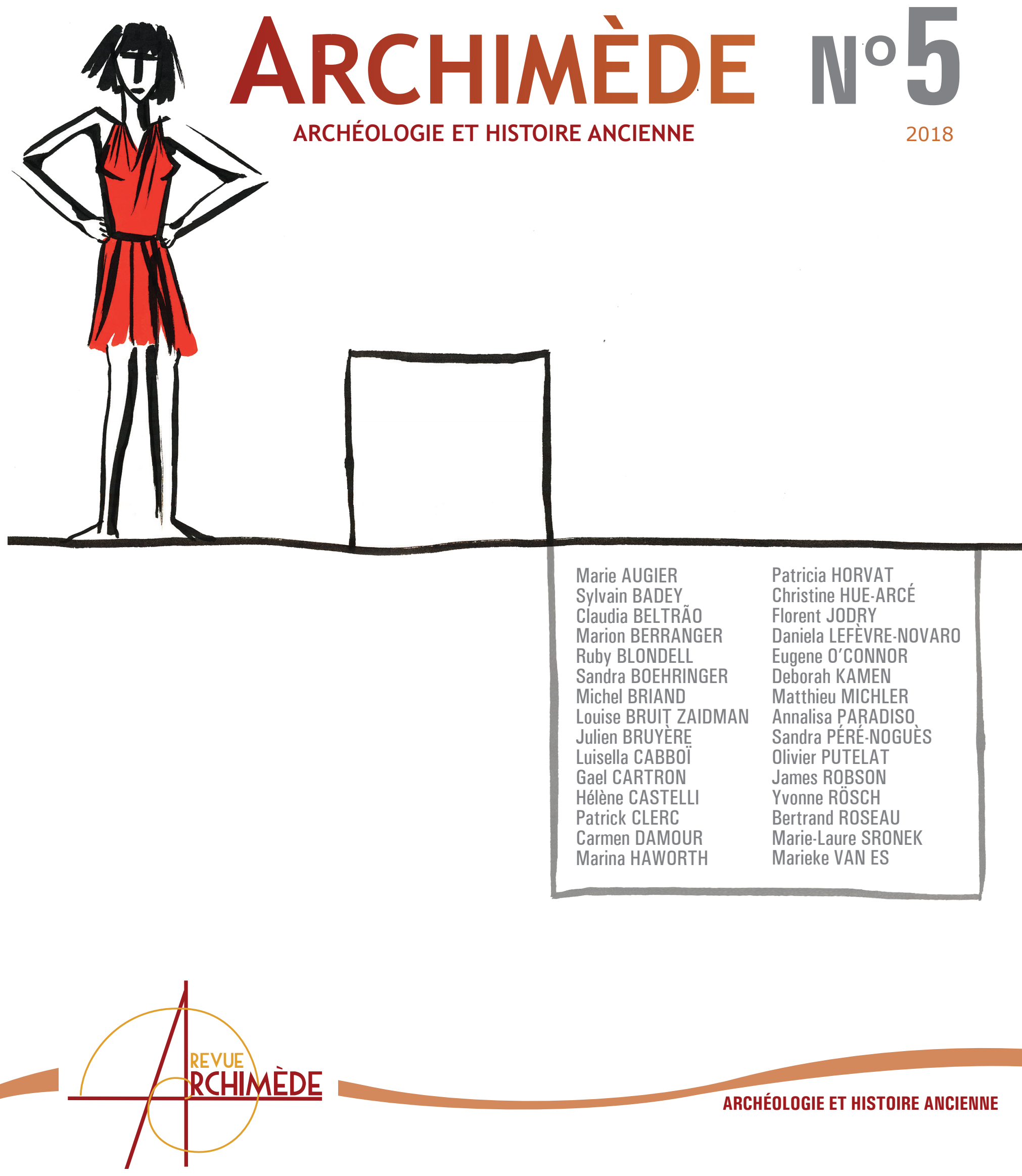


\section{COMITÉ DE RÉDACTION}

Loup BERNARD, MCF, Protohistoire (Université de Strasbourg)

Sandra BoEHRINGER, rédactrice en chef, MCF, Histoire ancienne, histoire des femmes et du genre (Université de Strasbourg)

Frédéric Colın, professeur, Égyptologie (Université de Strasbourg)

Sylvie Donnat, MCF, Égyptologie (Université de Strasbourg)

Clément FÉLIU, responsable d'opération, Protohistoire (INRAP)

Carole FÉVRIER, secrétaire de rédaction, chargée de ressources documentaires (AI, CNRS-UMR 7044)

Stephan FICHTL, professeur, Protohistoire (Université de Strasbourg)

Christian Jeunesse, professeur, Préhistoire (Université de Strasbourg)

Daniela LefÈvre-Novaro, professeure, Archéologie classique (Université de Strasbourg)

Doris MEYER, ingénieure de recherche (CNRS-UMR 7044)

Yannick MulleR, docteur, Histoire grecque (Université de Strasbourg)

Michel Humm, directeur de la revue et directeur du laboratoire, professeur, Histoire romaine (Université de Strasbourg)

Airton PolLINI, rédacteur en chef, MCF, Histoire grecque (Université de Haute Alsace)

Philippe Quenet, professeur, Archéologie de l'Orient ancien (Université de Strasbourg)

Anne-Caroline RENDU-LoIseL, MCF, Assyriologie et archéologie de l'Orient ancien (Unistra)

Jean-Jacques SchwiEN, MCF, Archéologie médiévale (Université de Strasbourg)

L'ensemble du comité de rédaction remercie chaleureusement Marie-José Morant pour son aide à la confection de ce numéro.

En couverture : $21^{\text {st }}$ Century Philaenis, illustration de Marjolaine Fourton.

Retrouvez tous les articles de la revue ARCHIMÈDE sur :

http://archimede.unistra.fr/revue-archimede

(creative $c$ commo (i) (2) (2) 
Laurent BaVAY (Université Libre de Bruxelles)

Audrey Bertrand (Université Paris-Est Marne-la-Vallée) Marie Besse (Université de Genève)

Luc Bourgeols (Université de Caen Basse-Normandie)

Nicola Cucuzza (Université de Gênes)

Madalina DANA (Université de Paris-1)

Marie-Pierre DAusse (Université de Paris-8)

Arianna Esposıto (Université de Bourgogne)

Guillaume Gernez (Université de Paris-1)

Adeline Grand Clément (Université Toulouse-Jean Jaurès) Valérie HuEt (Université de Brest)

Frédéric HuRLet (Université de Paris-10)

Michel KaZANSKI (CNRS-UMR 8167)

Brigitte LıON (Université de Lille-3)

Alain MARtin (Université libre de Bruxelles)
Dominic Moreau (Université de Lille-3)

Antonella PaUtasso (CNR-IBAM Catane)

Lilian Postel (Université Lumière Lyon-2)

Evelyne PrIOUx (CNRS-UMR 7041)

Vincent Puech (Université Saint-Quentin)

Isabelle RÉGEN (Université de Montpellier)

Umberto Roberto (Università Europea di Roma)

Emmanuelle Rosso (Université de Paris-4)

Martin Schönfelder (RGZM Mayence)

Margareta TENGBERg (Muséum national d'Histoire naturelle)

Jean TERrier (Université de Genève)

Yann TRISTANT (Macquarie University)

Emmanuelle VILA (CNRS-UMR 5133)

Youri Volokhine (Université de Genève)

Stéphanie WYLER (Université de Paris-7)

\section{COMITÉ SCIENTIFIQUE}

\author{
Maxence BAILly (Université d'Aix-Marseille) \\ Suzanne Bıckel (Université de Bâle) \\ Claude Calame (EHESS) \\ Philippe Collombert (Université de Genève) \\ Jean-Michel DAvID (Université de Paris-1) \\ Lukas DE BLoIs (Université de Nimègue) \\ Florence DuPONT (Université de Paris-7) \\ Axinia Džurova (Université de Sofia) \\ Dominique Garcia (Université d'Aix Marseille) \\ Jean Gascou (Université de Paris-4)
}

Anne Hauzeur (Institut royal des Sciences naturelles de Belgique)

\author{
Ralf von DEN Hoff (Université Fribourg-en-Brisgau) \\ Philippe Leveau (Université de Provence) \\ Enzo LiPPolıs ( $\dagger$ ) (Università La Sapienza Rome) \\ Pierre Maraval (Université de Paris-4) \\ Jean Margueron (EPHE) \\ Hartmut MATTHÄUs (Université Erlangen-Nuremberg) \\ Santiago MonTERo (Université Complutense de Madrid) \\ Christian SAPIN (CNRS-UMR 6298) \\ Evelyne Scheid-TIssinieR (Université de Paris-13)
}

Conception de I'interface : Catherine Duvette - Mise en ligne : Sabine Zinck - Logo et PAO : Shan Deraze vderaze@yahoo.fr

\section{MotS CLÉs de LA REVUE}

Archéologie

Préhistoire

Protohistoire

Antiquité

Antiquité gréco-romaine

Proche-Orient antique

Égyptologie

Moyen Âge
Proche-Orient

Moyen-Orient

Monde méditerranéen

Europe méditerranéenne

Europe occidentale

France

Italie

Grèce
Balkans

Archéozoologie

Papyrologie

Études coptes

Byzance

Illustration scientifique médiévale

Études byzantines 


\section{ARCHIMÈDE N5

1 DOSSIER THÉMATIQUE : HUMOEROTICA

108 ACTUALITÉ DE LA RECHERCHE : DES FEMMES PUBLIQUES. GENRE, VISIBILITÉ ET SOCIABILITÉ DANS L'ANTIQUITÉ GRECQUE ET ROMAINE

VARIA

185 Daniela LEFÈVRE-NOVARO

Phaistos, Dréros, Praisos : monuments publics et naissance de la polis en Crète

202 Matthieu MICHLER avec la collaboration de Sylvain BADEY, Marion BERRANGER, Luisella CABBOİ, Patrick CLERC, Florent JODRY, Olivier PUTELAT et Marieke VAN ES Ensilage et artisanat du fer entre le Hallstatt D3 et La Tène ancienne à Weyersheim (Bas-Rhin). Premiers résultats

230 Gael CARTRON, Bertrand ROSEAU et Julien BRUYÈRE

Le territoire de Warcq à l'époque romaine : résultats de six fouilles préventives (2012-2017) menées dans la partie nord du département des Ardennes, près de Charleville-Mézières 


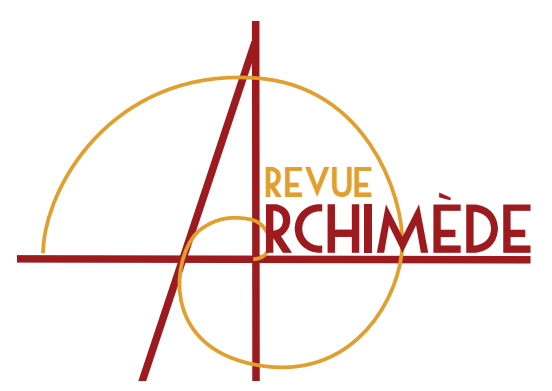

ARCHÉOLOGIE ET HISTOIRE ANCIENNE

\section{PHAISTOS, DRÉROS, PRAISOS : MONUMENTS PUBLICS ET NAISSANCE DE LA POLIS EN CRÈTE *}

\section{Daniela LEFÈVRE-NOVARO}

Professeur d'archéologie grecque Université de Strasbourg UMR 7044 Archimède

dlefevre@unistra.fr

\section{RÉSUMÉ}

La Crète est I'un des berceaux de la polis, comme I'indiquent les nombreuses données archéologiques qui montrent les connexions entre la mise en place des monuments publics et les débuts de l'organisation de la cité. L'analyse concernera trois poleis qui ont fait I'objet récemment de prospections et fouilles : Phaistos, Dréros et Praisos. Ces trois exemples permettent de visualiser les débuts d'un des phénomènes les plus marquants de la période archaïque en Grèce et surtout de mettre en exergue son origine très ancienne dans lî̂le (IX ${ }^{\mathrm{e}}$ siècle), conséquence sans doute de la réminiscence d'an-
MotS-CLÉS

Polis,

cité-État,

sanctuaire,

agora,

fortification. ciennes traditions politiques remontant à l'âge du Bronze mais aussi des influences provenant de la côte levantine.
The ancient Crete is one of the birthplaces of the polis, as attested by archaeological remains showing the connection between the first public buildings and the beginning of the city-state. In this paper I will study three poleis (Phaistos, Dreros and Praisos), recently concerned by new excavations and surveys. These examples allow us to focus on the origin of the city-state, which appeared here earlier than elsewhere in the Aegean world, probably because of the memories of ancient political customs of the Bronze Age in the island but also due to the influences from the Levant.
KeYWORDS

Polis,

city-state,

sanctuary,

agora, 


\begin{abstract}
* Cette contribution doit être considérée comme un complément à mon ouvrage sur la Messara (LEFÈVRE-NOVARO 2014) qui contient I'analyse détaillée d'hypothèses présentées ici en forme abrégée ainsi qu'une bibliographie exhaustive. Les abréviations chronologiques utilisées sont les suivantes : MR III A (Minoen récent III A); MR III B (Minoen récent III B) ; MR III C (Minoen récent III C) ; SMin (Subminoen) ; PGA (Protogéométrique ancien) ; PGM (Protogéométrique moyen) ; PGR (Protogéométrique récent) ; PGB (Protogéométrique B) ; G (Géométrique). Tous les siècles s'entendent avant J.-C. sauf indication contraire.
\end{abstract}

A Pierre Carlier, con affetto e riconoscenza

\section{INTRODUCTION}

L'île de Crète, tout en ayant conservé plus qu'ailleurs les traces du passé minoen et mycénien, a subi de nombreuses influences en raison de sa localisation au centre de la Méditerranée. Dans ce lieu de conservation d'anciens éléments culturels, un courant artistique « proto-orientalisant » se manifesta, par exemple, plus tôt qu'ailleurs, dès le IX $^{\text {e }}$ siècle [1]. Le mélange de traditionalisme et de nouveautés, dans une terre qui a su garder mémoire d'expériences multiformes accumulées pendant des millénaires, fait de cette île pendant le haut-archaïsme un véritable laboratoire pour l'élaboration de nouvelles structures politiques et sociales, précocement attestées par rapport au reste du monde grec. Sans entrer dans le débat historiographique concernant la définition de la polis [2], nous analyserons les données archéologiques et notamment les réalisations architecturales qui caractérisent les débuts de la cité en Crète. Il sera question notamment des sanctuaires urbains et extra-urbains, du premier lieu des réunions politiques (la proto-agora), des fortifications et du tissu urbain en général. Ces critères ne sont

[1] MaTthaüs 2011, p. 110 et 120 , note 61.

[2] Pour une analyse à ce propos, cf. LEFÈVRE-NOVARO 2007, p. 467-495. Voir aussi GEHRKE 2009, p. 395 pour la relation entre polis et État. Fondamentale est l'œuvre de Mogens Hansen et de son équipe (Copenhagen Polis Centre) : ils ont travaillé de 1993 à 2003 sur les thématiques inhérentes la polis (cf., entre autres, HANSEN 2006). certes pas exhaustifs mais ils peuvent être considérés comme prioritaires par rapport à d'autres déjà analysés ailleurs par d'éminents spécialistes. En effet la communauté scientifique ne s'accorde pas sur le rôle et l'importance qu'il faut attribuer à chacun de ces éléments dans la définition de la polis. En définitive la question est de savoir si I'un d'eux peut devenir un véritable marqueur archéologique de l'existence d'une cité grecque, comme I'avait proposé A. M. Snodgrass dans les années 1970 [3]. En l'absence de données précises sur les premiers lieux des réunions politiques des citoyens (sans aucun doute le critère le plus sûr pour identifier une polis), on a privilégié tantôt les fortifications, tantôt le sanctuaire de la divinité poliade, mais on ne peut attribuer une valeur absolue à aucune de ces réalisations architecturales. D'un côté, dans une situation de danger et d'insécurité, une enceinte peut être construite en relation avec un habitat qui n'est pas une polis. De l'autre, le rassemblement d'une communauté humaine autour de cultes communs est un phénomène bien connu ailleurs que dans les cités grecques [4]. Ces deux critères ne peuvent donc pas être utilisés de manière isolée ; ils doivent faire partie d'un

[3] Pour une reprise de ces thèmes de recherche, cf. SNODGRASS 1993. Une bonne mise au point récente est celle de CRIELAARD 2009.

[4] Pour la période minoenne, il suffit de songer aux kouloures et au sanctuaire protopalatial de la cour I du palais de Phaistos et au sanctuaire du quartier XVIII du palais de Mallia dont I'entrée donne vers l'extérieur du palais, vers la ville. 
faisceau d'indices concordants, notamment en association avec les attestations des institutions politiques et sociales qui restent fondamentales pour identifier la naissance de la polis.

Avant de prendre en compte l'archéologie [5], il faut souligner l'importance des données épigraphiques concernant les cités crétoises au début de leur développement. Les inscriptions en effet attestent le fonctionnement de ce type d'organisation politique dans l'île au moins dès la fin du VIII ${ }^{\mathrm{e}}$ siècle. L'indication chronologique la plus explicite vient d'une inscription découverte dans la grande citerne près du temple d'Apollon à Dréros : il s'agit de la plus ancienne loi grecque inscrite [6], mentionnant pour la première fois le terme de polis. En effet dans ce texte la polis (vraisemblablement la communauté des citoyens) réglemente le mandat des Cosmes (les magistrats les plus importants de la cité) pour éviter le renouvellement des mandats et, de toute évidence, I'instauration d'un pouvoir personnel. Parmi les jureurs de la loi sont mentionnés le collège des Cosmes, les Damioi et les Vingt de la cité [7]. Tout en laissant de côté le problème des compétences spécifiques de chaque groupe de magistrats, il est clair que, vers le milieu du VII ${ }^{\mathrm{e}}$ siècle, la cité de Dréros s'était déjà dotée d'une organisation institutionnelle complexe (trois collèges de magistrats ayant sans doute des compétences différentes) au sein de la communauté des citoyens qui exerce la souveraineté. Vraisemblablement cette polis fonctionnait depuis assez longtemps (deux générations ?) pour que les Drériens se rendent compte du danger de la mise en place d'un pouvoir personnel et qu'ils prennent les dispositions législatives pour l'éviter. D'après les données épigraphiques, on peut donc supposer qu'en Crète les institutions politiques réglaient et organisaient la vie de la communauté au moins à partir de la fin du viıI ${ }^{\mathrm{e}}$ siècle.

Pour mener à bien cette analyse qui vise à préciser les caractéristiques, les fonctions et la chronologie des principales réalisations architecturales qui jalonnent la mise en place de la polis, nous prendrons en considération trois cités crétoises qui sont autant d'exemples significatifs en raison d'avancées récentes dans les recherches et de leur localisation dans trois régions différentes de l'île : Phaistos (Messara, Crète méridionale), Dréros (Mirabello, côte septentrionale) et Praisos (Crète orientale).

\section{LE CHOIX DU SITE}

En premier lieu, il faut souligner l'analogie dans l'emplacement de ces trois cités, installées sur des collines aux pentes escarpées par endroits, mais dans aucun cas imprenables. La raison principale du choix de ces lieux n'est donc pas à rechercher dans la nécessité de se défendre [8], mais plutôt dans la position stratégique, sur une éminence, qui permet d'avoir une bonne visibilité et un contrôle optimal sur le territoire : Phaistos sur la partie occidentale de la plaine de la Messara ; Dréros sur le couloir naturel qui relie le golfe de Mirabello et la plaine de Milatos ; Praisos dans la vallée du fleuve Sitia qui forme avec le torrent qui débouche au sud, près de Pilalimata, I'une des voies de communication privilégiées entre le nord et le sud de la Crète orientale. Pour expliquer le choix de ces localisations, outre les critères économiques liés à I'exploitation du territoire (agriculture, élevage, artisanat) [9], il faut aussi tenir compte de la continuité de fréquentation de l'Âge du Bronze à l'Âge du Fer, aspect fondamental dans un contexte insulaire.

Phaistos (fig. 1), siège de l'un des plus importants palais minoens, fut habité du Néolithique final au MR III C récent, donc tout au long de la période minoenne [10]. Les données concernant le SMin et les phases PGA-PGM se limitent à des contextes funéraires, mais il pourrait s'agir simplement de la conséquence de l'état de la recherche qui a privilégié pendant des décennies la période minoenne. À partir
[5] Pour une analyse récente des données archéologiques concernant la formation des poleis en Crète, cf. WALLACE 2010, notamment p. 233-342.

[6] Sur les lois crétoises et sur ce texte en particulier, cf. Gagarin \& PERLMAN 2016, p. 199-207.

[7] Loi constitutionnelle de Dréros contre l'itération du cosmat : « La cité a décidé : quand quelqu'un aura été Cosme, de dix ans le même personnage ne sera plus Cosme ; s'il venait à être Cosme, dans tous les cas où il prononcerait un jugement, lui-même devra l'amende au double ; il sera maudit et privé de ses droits civiques aussi longtemps qu'il vivra et tout ce qu'il aura fait comme Cosme sera nul. Jureurs : le collège des Cosmes (?), les Damioi et les Vingt de la cité ». Pour la traduction, cf. VAN EfFEnTERRE \& RuzÉ 1994, p. 306-308 (n 81), chronologie vers 650 ; même datation dans GuARDUCCI 1967 , p. 187-188 et dans JEFFERY 1990, p. 315.

[8] C'est I'hypothèse de NowICKI 2000 qui a été fort critiqué à ce propos.

[9] Ces thématiques ont déjà été développées dans LEFÈVRE-Novaro 2007, p. 470-471 (exploitation en général), p. 477-479 (le cas de Phaistos).

[10] Pour un tableau chronologique de ces périodes, cf. LefÈvRe-Novaro 2014, 1, p. 29. 


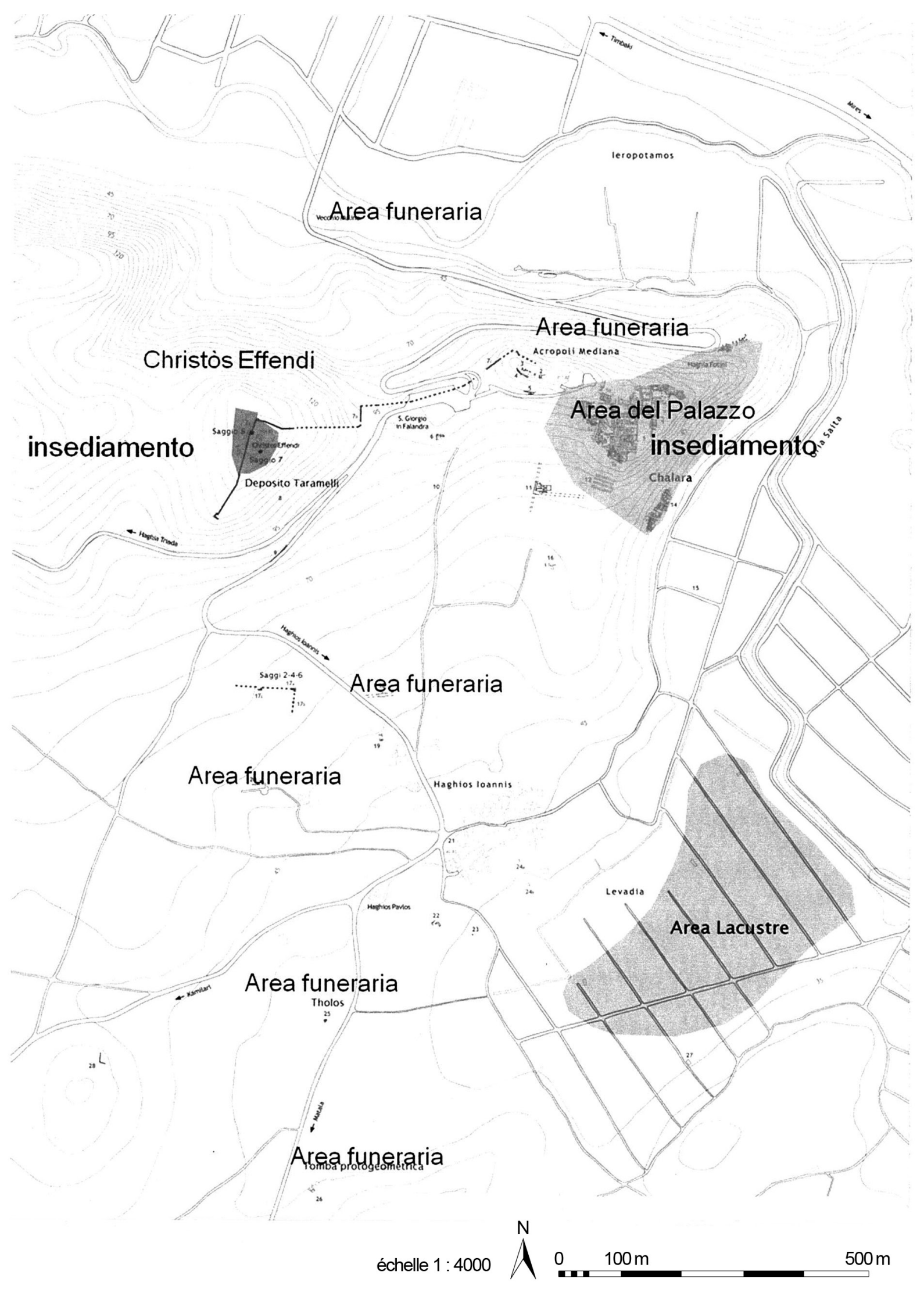

Figure 1 : plan de la ville de Phaistos (d'après Longo 2015, pl. IV). 


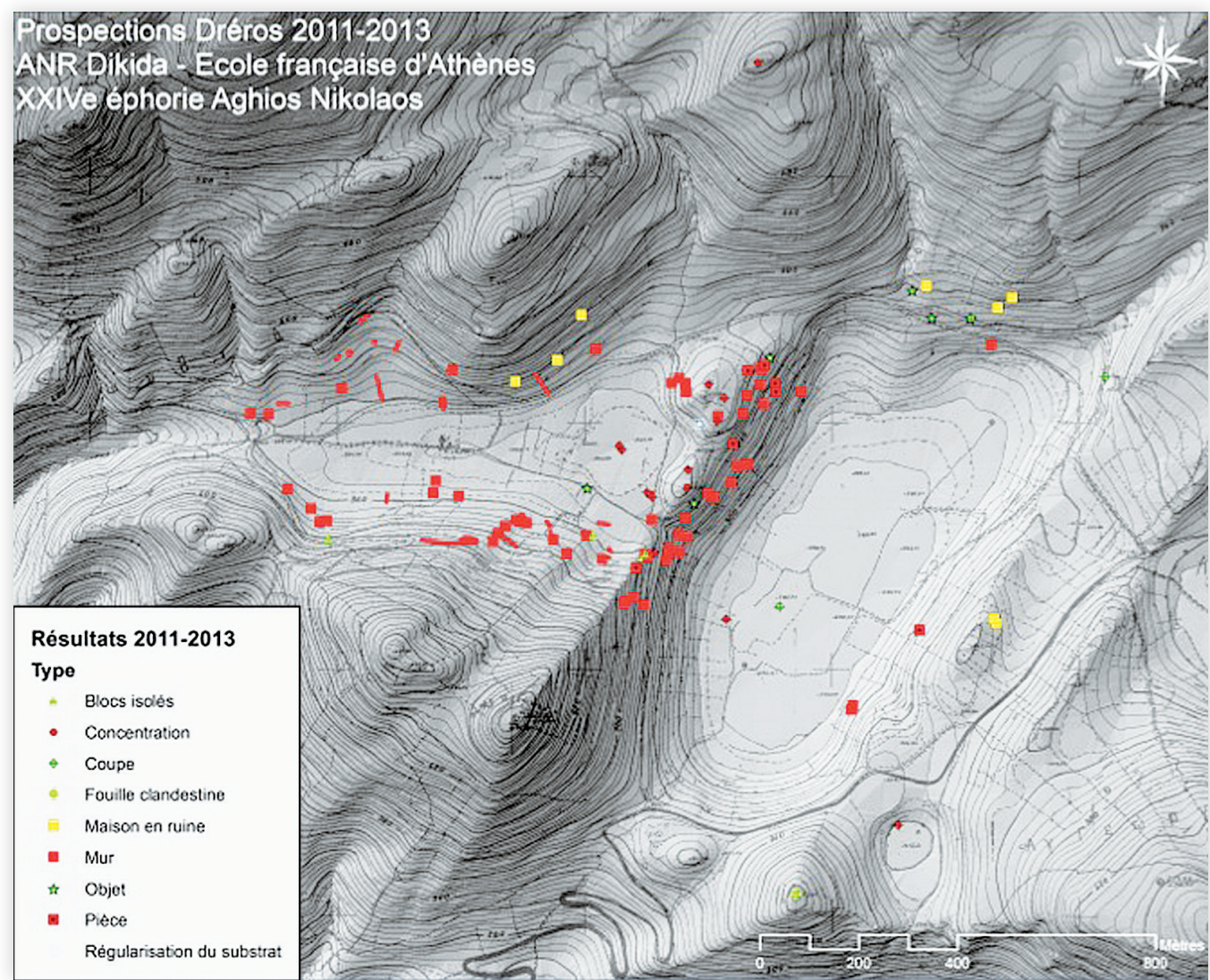

Figure 2

Plan du territoire au nord de la ville de Dréros avec les résultats des deux campagnes de prospection (2011 et 2013), ANR DIKIDA.

Élaboration

N. Carayon. du PGR en tout cas, le site est de nouveau fréquenté par une importante communauté humaine organisée kata komas [11].

Pour le territoire autour de la cité de Dréros [12], la prospection qui a eu lieu en 2011 et 2013 (fig. 2) a révélé des traces de fréquentation de l'Âge du Bronze non seulement sur l'éminence de Képhali Limnes [13], à l'est de Dréros, mais aussi dans la petite vallée au nord du site (pieds de marmites tripodes) et sur les pentes septentrionales du poljé de Trivaxona. Cette dernière zone a restitué notamment deux kylikes fragmentaires et un tesson appartenant sans doute à un deep bowl, outre des pithoi et de la céramique commune [14]. La zone de la future cité sur la double acropole (fig. 3) fut apparemment fréquentée plus tard, à partir du MR III C-Smin, comme I'indiquent les tessons découverts par K. Nowicki sur les plus hautes pentes de I'une des deux acropoles [15] ainsi que la chronologie de la tombe $n^{\circ} 1$ de la nécropole fouillée par $\mathrm{H}$. Van Effenterre au nord du site, près de I'église d'Haghios Georghios [16]. La table à offrandes minoenne en stéatite, découverte dans la pièce IX de l'édifice au sud du temple d'Apollon, à proximité de l'agora de Dréros, doit être considérée probablement comme une antiquité réutilisée dans un contexte plus récent [17].

Le site de Praisos (fig. 4), fréquenté depuis le Néolithique final et occupé en partie à la période minoenne, se développa surtout à partir du MR III [18] : parmi les tombes à tholos, l'une est datée du MR III A et plusieurs sépultures remontent
[11] La continuité de fréquentation sur la longue durée est notamment avérée pour la colline du palais minoen : LA ROSA 2010, p. 583 (périodes néolithique et minoenne); LEFÈVRE-Novaro 2014, 2, p. 209-244 (du MR III à l'époque orientalisante). Pour un possible habitat géométrique aussi sur la colline de Christos Effendi, cf. LONGO 2015, p. 164.

[12] Synthèse récente sur cette ville dans GAIGNEROTDRIESSEN 2016, p. 221-244.

[13] Pour une description récente du site, ibid., $p$. 218-221 et p. 235 , fig. 11.4 pour la localisation de Dréros et du poljé de Trivaxona.

[14] La prospection a été effectuée par l'équipe du programme ANR DIKIDA, dirigé par D. Lefèvre-Novaro et financé de 2011 à 2014 par l'Agence Nationale de la Recherche. Le mobilier découvert est en cours d'étude. Je remercie V. Zographaki et A. Farnoux pour l'aide apporté lors de la réalisation de la prospection.

[15] NowICKI 2000, p. 173. Le spécialiste parle de la colline de Dréros au singulier (« [...] at many spots on the summit and highest slopes of the hill $[\ldots] \gg)$, mais le site est caractérisé par une double acropole ; par conséquent le lieu exact de découverte des tessons reste inconnu.

[16] Tsipopoulou 2005, p. 62 ; VAN EffenterRe 2009, p. 59-62.

[17] Demargne \& Van Effenterre 1937, p. 17, fig. 10. [18] WHITLEY et alii 1999, p. 224-238. 


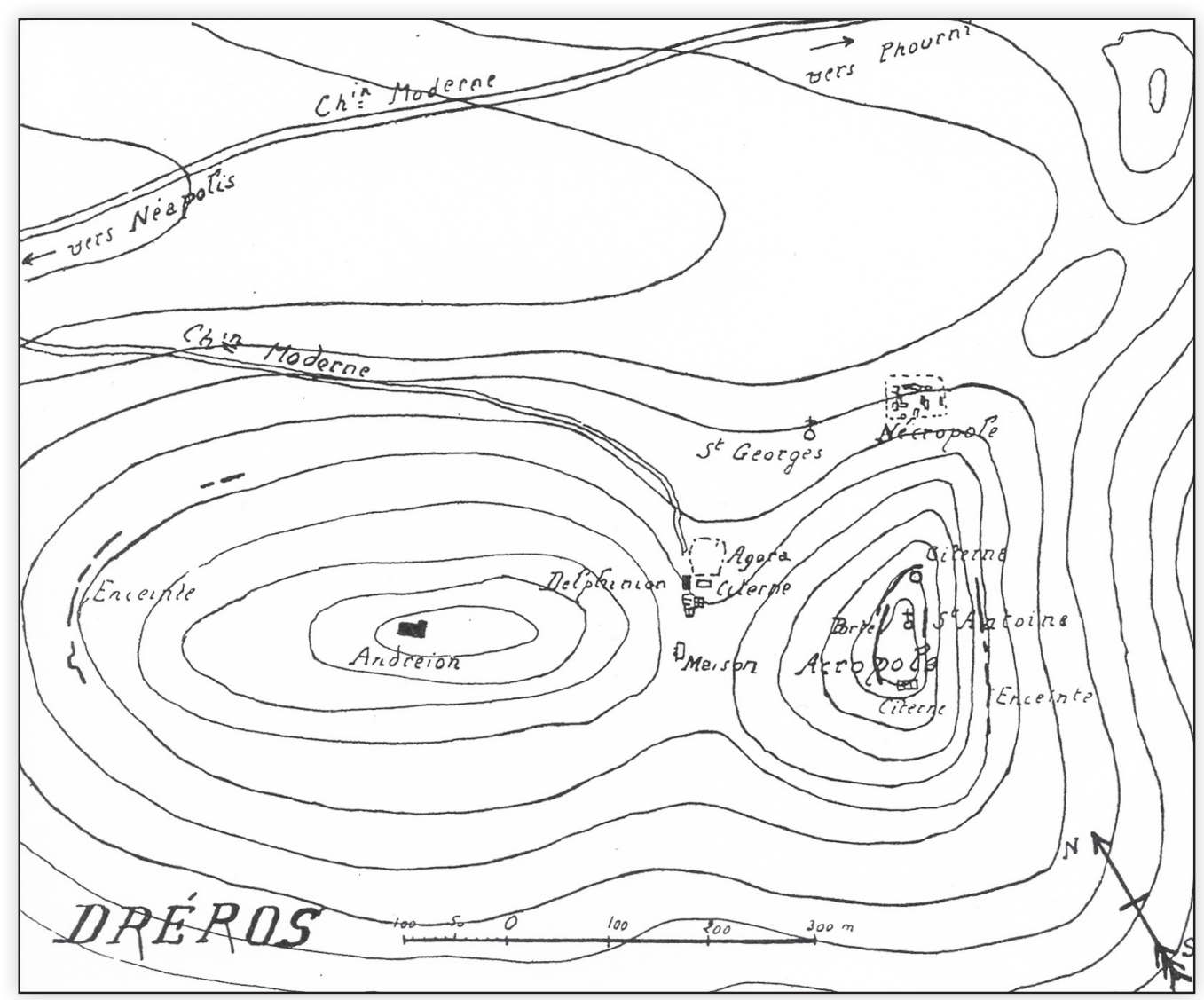

au MR III B et au MR III C [19]. Lors de la prospection dans la zone de la ville, on a découvert des tessons datés des périodes allant du MR III C à l'Orientalisant ; des tombes géométriques se trouvent à proximité du site. Il faut en outre mentionner l'établissement sur la hauteur de Kalamafki Kipia, situé à l'est du site, qui fut fréquenté à partir de la phase finale du MR III B et abandonné vers 900 (PGM) [20].

En définitive les trois sites en question, fréquentés selon des modalités différentes, sont caractérisés par une localisation similaire, sur des collines qui dominent le territoire ; la continuité d'habitat est attestée à Phaistos depuis le Néolithique final et pour Praisos au moins depuis le MR III [21], comme

[19] Belli 1991, p. 440 ; WhitLey 1998 ; WhITLEY 2006, p. 600. Pour une tombe datée du MR III C découverte au lieu-dit Photoula, cf. To Ergon tis Arkaiologikis Etaireias kata to 1960 (1961), p. 212, fig. 242.

[20] J. Whitley souligne l'absence de tessons datés du PGR et du G : WhITLEY 1998, p. 32.

[21] Les vestiges minoens découverts sur le site sont d'interprétation controversée et certaines phases sont peu représentées (Minoen ancien et période néopalatiale).

[22] Les spécialistes considèrent que les fortifications ne constituent pas l'élément le plus caractéristique de la d'ailleurs probablement aussi dans le cas du territoire de Dréros, bien que les données préliminaires de la prospection restent à vérifier.

\section{LES FORTIFICATIONS}

Parmi les réalisations architecturales qui peuvent caractériser la polis [22], mais qui n'ont laissé que de rares traces à l'époque archaïque en Crète, on peut citer les fortifications. En effet le site de Praisos en serait totalement dépourvu [23], tandis que l'enceinte identifiée par $\mathrm{S}$. Marinatos à Dréros (fig. 3) [24], autour de l'acropole est et sur les pentes septentrionales et occidentales de l'acropole

cité grecque, bien que pouvant devenir imposantes dans certains cas : LAFON et alii 2011, p. 59.

[23] Coutsinas 2013, p. 292-294. Mais des fortifications, probablement datées des phases MR III B-C, ont été identifiées par l'équipe de J. Whitley autour du sommet de la première acropole : cf. Alusik 2007, p. 50 .

[24] MARINATOS 1936, p. 217. Voir aussi DemaRgne \& VAN EFFENTERRE 1937, p. 7 qui soulignent déjà le fait que : « [...] si l'élévation en est sûrement moderne, le plan et les fondations sont peut-être antiques ». Les fortifications de Dréros feront I'objet d'une nouvelle analyse approfondie dans le cadre de la reprise des recherches sur le site. 
ouest, serait d'après N. Coutsinas [25] fort problématique, puisqu'en partie elle ne daterait que de l'époque byzantine (acropole est : présence de briques et chaux). À Phaistos, l'enceinte mise au jour par A. Minto [26] remonte probablement au IV siècle, comme l'ont confirmé les recherches récentes de l'équipe de F. Longo [27]. Cependant un tronçon de mur plus ancien et probablement à fonction défensive est en partie encore visible au sommet de l'acropole médiane, du côté ouest (fig. 1). Son interprétation est incertaine, tant du point de vue du parcours que pour sa chronologie (PG ? G ?) [28], mais sa forme curviligne avec une sorte de bastion à l'extrémité nord, son élévation et son épaisseur $(2,80 \mathrm{~m})$ empêchent de l'interpréter comme le mur d'un bâtiment ou un mur de terrassement. L'hypothèse qu'il s'agisse de l'enceinte géométrique de la cité, un lieu d'ultime refuge sur I'acropole médiane, englobée peut-être au IV siècle dans la nouvelle fortification, pourrait être mise en relation avec la rivalité séculaire entre Phaistos et Gortyne [29].

En l'état actuel de nos connaissances, aucune des trois cités ne semble donc avoir attribué un rôle fondamental à la fortification au moment de la mise en place de la polis [30], sauf peut-être dans le cas du mur de l'acropole médiane de Phaistos qui reste toutefois difficile à dater.

\section{LES SANCTUAIRES}

Dans les cités crétoises, comme dans le reste du monde grec intéressé par la diffusion de la polis, le sanctuaire joue un rôle prédominant dans la définition de ce nouveau système politique [31].

À Phaistos, l'activité cultuelle dans le sanctuaire de la Mégala Mater [32] (fig. 5) est attestée dès les dernières décennies du VIII ${ }^{\mathrm{e}}$ siècle grâce à la découverte de boucliers et chaudrons fragmentaires en bronze, cachés sous le sol du pronaos du temple actuellement

[25] COUTSINAS 2013, p. 208-209.

[26] MINTO 1921-1922.

[27] LONGO 2015, p. 168.

[28] LeVi 1956, p. 241 ; Lefèvre-Novaro 2014, 1, p. 176 et 2 , p. 227-228.

[29] LefÈVRe-Novaro 2014, 2, p. 227-228 et 242.

[30] Même conclusion en général dans WALLACE 2010, p. 284.

[31] L'ouvrage de F. DE PoLignaC (1991) est fondamental pour toutes ces questions, notamment à propos de l'importance des sanctuaires extra-urbains.

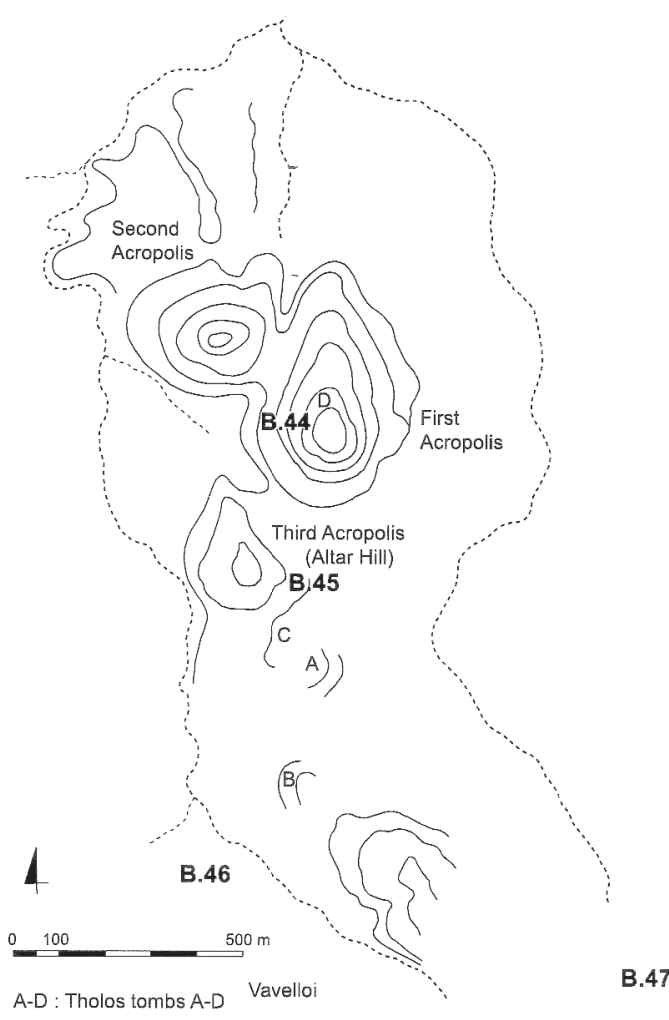

Figure 4 : plan du site de Praisos (d'après Prent 2005, fig. 52).

visible. Ce dernier fut construit dans le dernier quart du VII ${ }^{\mathrm{e}}$ siècle [33], mais il fut peut-être précédé par un autre édifice de culte en matériaux périssables dans lequel ces objets votifs étaient consacrés ; ses traces pourraient avoir été effacées par la longue fréquentation de la zone du sanctuaire [34].

Il faut en outre mentionner la reprise du culte au PGB dans le sanctuaire extra-urbain d'Haghia Triada, situé environ trois kilomètres à l'ouest de Phaistos [35]. Toutefois ce lieu de culte n'est pas caractérisé par un temple. Les rituels comportaient la déposition d'ex-voto, essentiellement des figurines en terre cuite et parfois en métal, près des ruines de la villa minoenne abandonnée. Ces vestiges deviennent en quelque sorte le lieu

[32] Pour une mise au point sur le sanctuaire, avec bibliographie, cf. LEFÈVRE-NOVARO 2009, p. 565-570.

[33] LA ROSA 2000, p. 32.

[34] La même remarque est d'ailleurs valable pour les temples urbains de Gortyne et Prinias : ces édifices présentent une décoration sculptée au cours de la seconde moitié du VII ${ }^{\mathrm{e}}$, voire vers la fin de ce siècle, mais rien n'empêche de supposer qu'ils furent précédés par d'autres bâtiments à fonction cultuelle en matériaux périssables, surtout au vue des dépôts de fondation remontant au PGB (LeFÈVRE-Novaro 2014, 1, p. 243).

[35] LeFÈVRE-Novaro 2014, 2, p. 262-265. 


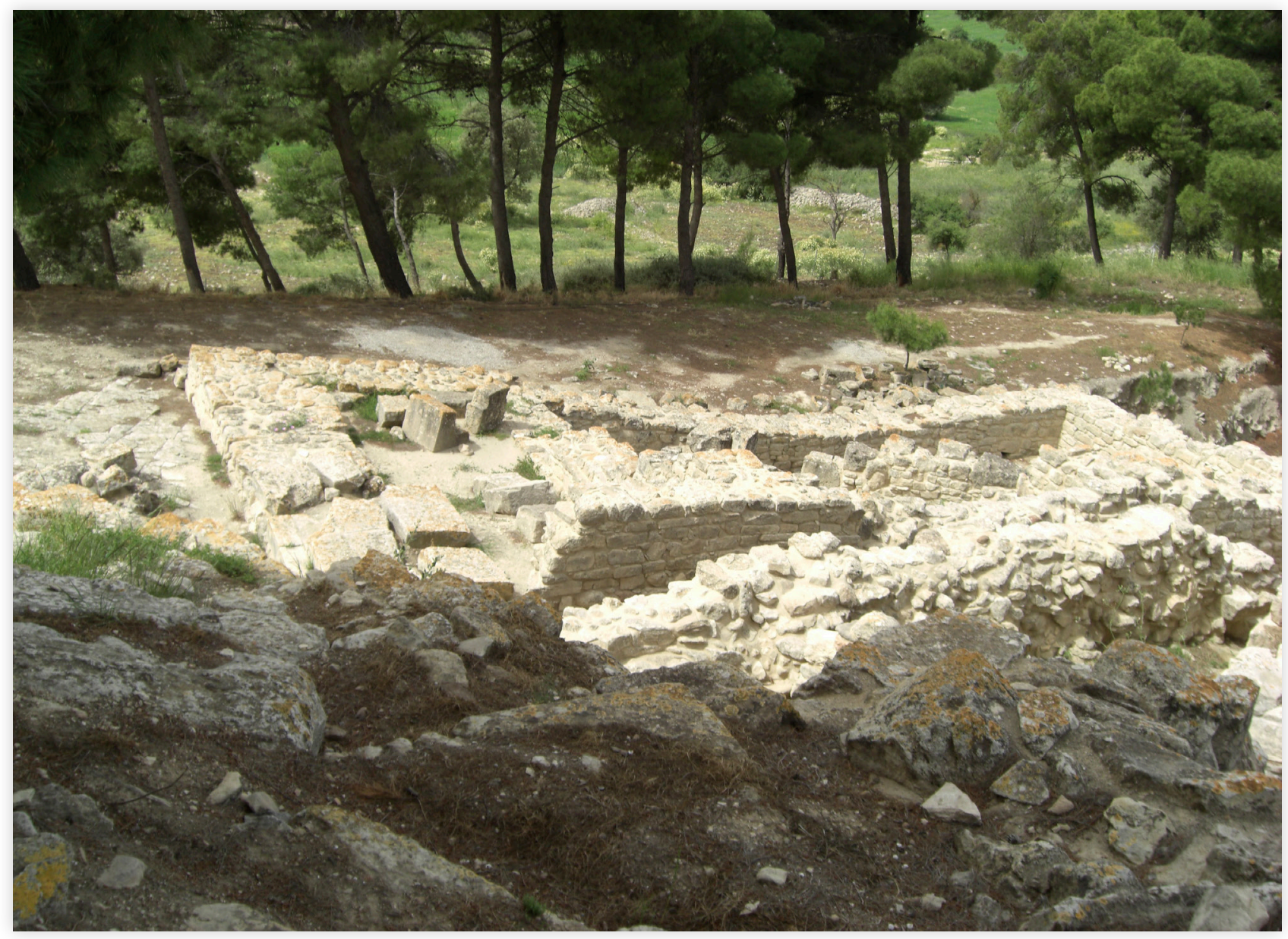

Figure 5 : le temple de la Mégala Mater, vu du nord. Photo : D. Lefèvre-Novaro.

d'attraction de cérémonies mémorielles, un phénomène bien connu dans le monde égéen pendant le haut-archaïsme [36].

Dréros est la seule cité parmi les trois analysées qui fut dotée d'un temple construit en partie en pierre entre les dernières décennies du viII e et le milieu du VII ${ }^{\mathrm{e}}$ siècle, ce bâtiment étant utilisé ensuite jusqu'à la destruction de la ville à l'époque hellénistique (fig. 6). Bien qu'une reprise de l'étude architecturale

[36] Cf. Camberlein 2015 pour des considérations préliminaires sur le rôle des keimelia en Crète.

[37] Marinatos 1936, p. 219-232 ; PRENT 2005, p. 284-289.

[38] ZOGRAPHAKI \& FARNOUX 2014, p. 106 pour les kalathoi G-O découverts aussi sur l'acropole ouest.

[39] ROLLEY 1994, p. 112.

[40] LeFÈVRE-Novaro 2014, 2, p. 283-286.

[41] LEFÈVRE-NOVARO 2014, 1, p. 243-245.

[42] ZOGRAPHAKI \& FARNOUX 2014, p. 106.

[43] MARINATOS 1936, p. 253-254. On développera ailleurs le sujet très intéressant de l'andreion : cf. LEFÈVRE-Novaro 2014, 1, p. 162-165 et l'excellent travail de MONTECCHI 2007. et de la stratigraphie du secteur du temple s'avère nécessaire, on peut déjà souligner les indices en faveur de I'hypothèse d'une chronologie haute de l'édifice : la découverte à l'intérieur du naos de céramique datée du GR et de l'époque archaïque [37], dont de nombreux kalathoi [38], de figurines en terre cuite archaïques et de trois statues de culte en bronze, représentant la triade apollinienne, datées par C. Rolley de la fin du VIII ${ }^{\mathrm{e}}$ siècle [39] ; le terminus ante quem représenté par les inscriptions archaïques tombées dans la citerne mais faisant partie à l'origine vraisemblablement du mur oriental du temple et dont les plus anciennes remontent à 650 ; enfin la typologie architecturale du temple à foyer central, attestée à Kommos à partir de 800 (temple B) [40] et connue grâce à d'autres exemples édifiés en Crète au cours du VII ${ }^{\mathrm{e}}$ siècle [41].

Un autre temple fut construit à Dréros au cours du VII ${ }^{\text {e }}$ siècle sur l'acropole ouest (fig. 7), pour monumentaliser un sanctuaire établi au moins dès la fin du VIII ${ }^{\mathrm{e}}$ siècle [42]. L'étude en cours de ces importants vestiges, qui autrefois ont été identifiés comme un andreion par S. Marinatos [43], permettra d'avoir un aperçu plus clair des sanctuaires de la 


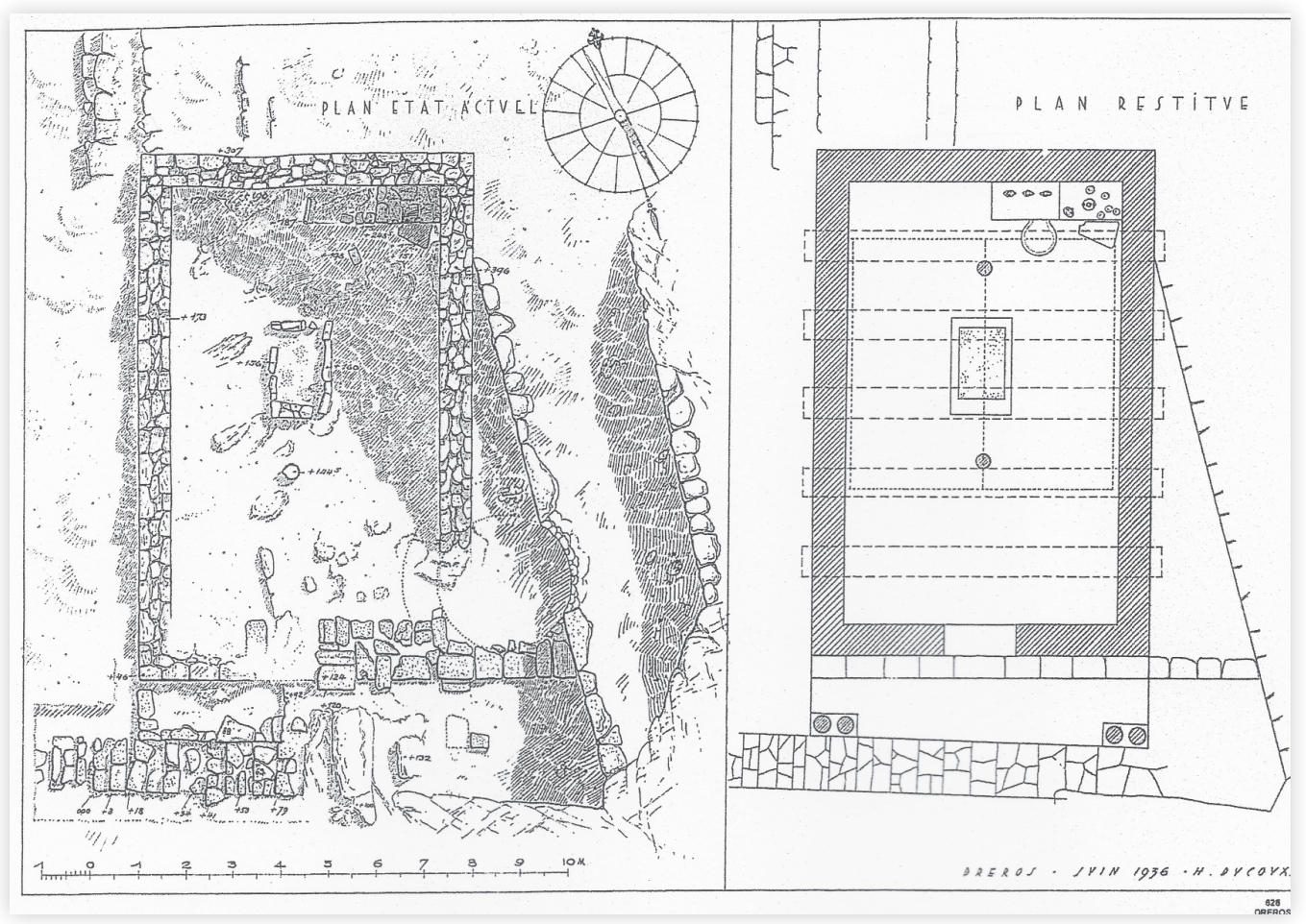

Figure 6

Plan du temple d'Apollon

Delphinios, relevé pierre à pierre et plan au sol restitué (d'après Marinatos 1936, pl. XXVII).

ville pendant le haut-archaïsme. On peut d'ores et déjà souligner les nombreuses dédicaces d'armes, pour la plupart défensives (casques, cuirasses, mitrai, boucliers, etc.), témoignages de la participation des élites aux cérémonies. Ces ex-voto pourraient d'ailleurs être mis en relation avec le culte d'Athéna Poliouchos, déesse qui trouverait ainsi sa place sur l'une des deux acropoles de la ville et qui est attestée par les inscriptions dont le Serment des Drériens, découvert juste à côté [44].

À Praisos, le sanctuaire sur la troisième acropole ou colline de l'autel (fig. 4), fondé au viII ${ }^{\mathrm{e}}$ siècle, est caractérisé par un autel et un important dépôt votif, tandis qu'aucun temple ne vit jamais le jour. Le sanctuaire en plein air abrita des constructions seulement à partir du ve siècle, mais il ne s'agit que d'un temenos avec quelques pièces annexes qui entourent I'autel [45]. J. Whitley rappelle en outre la découverte de plaquettes votives en terre cuite, datées à partir de 700, dans les sanctuaires des sources de Vavelloi et de Mesamvrysis [46].

La mise en place du sanctuaire est donc précoce dans ces poleis (dernières décennies du viıI ${ }^{\mathrm{e}}$ siècle) et accompagne la structuration de la communauté politique, mais elle n'implique pas forcément la construction d'un temple, en tout cas archéologiquement identifiable : le cas du naos de Dréros, construit en partie en pierre, est parmi les plus anciens attestés dans les cités crétoises. D'un point de vue architectural, il présente des caractéristiques typiques de I'île où la forme du temple grec canonique eut du mal à s'imposer, même aux époques classique et hellénistique. Il s'agit en effet d'un simple oikos rectangulaire, ouvert au nord-nord/est et abritant un foyer au centre ; ses dimensions sont réduites, mais des pièces annexes étaient adossées du côté nord-ouest, selon la tradition architecturale minoenne des bâtiments à pièces agglutinées. Ce type de temple, qu'on définit généralement comme hybride, est diffusé en Crète pendant le haut-archaïsme sans qu'on puisse pour l'instant en préciser les origines. Il avait de toute évidence deux fonctions : maison des dieux et lieu destiné au sacrifice et à la cuisson de la viande, peut-être même salle de banquet. Cette dernière fonction n'est en réalité attestée que par la découverte, à l'intérieur de l'édifice de Dréros, de coupes, d'ossements et d'une hypothétique banquette du côté nord. Les dimensions réduites du bâtiment semblent impliquer un accès restreint aux cérémonies, sans doute réservées aux plus hauts magistrats. Aux origines, le temple aurait ainsi joué le rôle de prytanée [47] tandis que le reste de la population participait probablement au sacrifice en s'assemblant dans le

[44] IC I, IX, 1, lignes 22-23. Cf. aussi D'ACUNTO 2002-2003, p. 55-58.

[45] Bosanquet 1901-1902, p. 254-259; Prent 2005, p. 304-306

[46] WHITLEY 2006, p. 606.

[47] D'ACUNTO 2002-2003, p. 47-48. Rappelons toutefois qu'à l'époque hellénistique le prytanée de Dréros était un édifice séparé du temple : IC I, IX, 1 , lignes $15-16$ et 117 . 
vaste espace au nord-est du temple, là où l'agora d'époque hellénistique est aujourd'hui visible. Le rôle politique de ce temple, en tant qu'epiphanestatos topos, est en outre assuré par la présence sur ses murs de nombreuses inscriptions archaïques, concernant des textes de loi, découvertes tombées à l'intérieur de la citerne aménagée à l'époque hellénistique juste à l'est du temple. Il s'agit d'un usage connu ailleurs en Crète et qui serait intéressant d'analyser dans le détail, par exemple dans les cas du Pythion de Gortyne et du temple d'Axos, en le mettant en relation avec les personnalités divines vénérées [48].

En ce qui concerne les attestations iconographiques, il faut rappeler l'importance des images sur la ceinture en bronze découverte dans la nécropole de Fortetsa (Cnossos) [49], probablement I'œuvre d'un artisan d'origine proche-orientale, et sur le relief en calcaire de Chania qui fait sans doute partie de la décoration d'un édifice, peut-être un temple, hypothèse qui rendrait l'attestation encore plus significative [50]. Ces scènes montrent que, au cours du viII ${ }^{\mathrm{e}}$ siècle en Crète, la représentation de la ville se résumait probablement au temple, tandis que dans les documents iconographiques proche-orientaux contemporains, par exemple, on trouve la représentation de la citadelle fortifiée [51]. Puisque le temple était le monument public qui représentait à lui seul les agglomérations de l'époque en Crète, on peut donc envisager I'hypothèse que, lorsque les données archéologiques font défaut, ce manque d'informations ne soit dû qu'à la nature périssable des matériaux de construction ou bien au hasard des découvertes.

D'après J. Whitley [52], le cas de Praisos serait différent : en effet, à aucun moment le développement du sanctuaire sur la colline de l'autel ne comporta la construction d'un temple, comme si l'emplacement sur un sommet, bien visible dans le paysage, suffisait à mettre en valeur le plus ancien lieu de culte de la ville. De ce point de vue, le sanctuaire de Praisos rappelle les sanctuaires de sommet minoens, y compris d'ailleurs pour la pratique d'allumer des

[48] Pour des considérations intéressantes à ce propos, voir PERLMAN 2004, p. 181-197.

[49] RoLLey 1994, p. 124-125, fig. 103-104. Sur la ceinture de Fortetsa, voir aussi D'AcunTo 2013.

[50] Le relief est haut de $39 \mathrm{~cm}$. Je remercie $\mathrm{H}$. Matthäus pour cette suggestion.

[51] D'ACUNTO 2002-2003, p. 33-34.

[52] WHITLEY 2006, p. 608-614.

[53] Duнoux 1982, p. 63-85. bûchers sur une éminence et de jeter les ex-voto (dans ce cas, des armes) dans les crevasses de la roche. L'absence d'un édifice de culte serait donc dans ce cas un choix intentionnel lié à l'origine étéocrétoise de l'établissement, où cinq inscriptions en langue non grecque furent découvertes [53], et à la volonté de conserver les usages ancestraux.

\section{L'AGORA}

Ces considérations sur les fonctions du sanctuaire à l'origine de la polis amènent à s'intéresser aux rares informations disponibles sur l'agora à la même époque. Cet espace emblématique de la cité grecque, véritable symbole de la participation directe des citoyens au fonctionnement des institutions politiques et sociales, se développa au fur et à mesure des évolutions de la communauté, notamment en Crète où les liens claniques sont encore très marqués pendant les phases les plus anciennes de la polis [54]. Homère [55] rappelle que, aux origines, l'agora n'était qu'un espace en plein air, sans installations particulières : un cercle de pierres polies suffisait pour accueillir les anciens (gerontes). Ce n'est qu'au fur et à mesure de l'évolution des institutions que des bâtiments et des structures vinrent encadrer et monumentaliser la place publique. Qu'en est-il alors des plus anciennes agorai des trois cités crétoises étudiées ici ? Pour Phaistos, les recherches récentes de l'équipe de $\mathrm{F}$. Longo et les carottages effectués grâce au programme ANR Dikida ont fourni des éléments nouveaux : d'un côté il est aujourd'hui avéré qu'une zone humide s'étendait au pied de la colline du palais, à proximité du quartier de Chalara (fig. 1), là où on avait imaginé la présence de l'agora en raison de la découverte d'une inscription archaïque la mentionnant ainsi que des fondations d'édifices de dimensions importantes [56]. De I'autre, la prospection systématique dans la zone de la ville de Phaistos a mis en évidence un « vide » d'attestations entre l'acropole médiane et le village d'Haghios Ioannis, dans les secteurs central et
[54] Il suffit de songer aux nombreuses tombes multiples crétoises, utilisées de génération en génération pendant le haut-archaïsme, qui ont été découvertes par exemple à Gortyne (tholos d'Haghios Georghios : LefèVRENovaro 2014, 2, p. 181 ; ANZALONE 2015, p. 31-32), à Eleutherna (par exemple la tombe A1K1 : STAMPOLIDIS 2004, p. 122-124), à Cnossos, etc.

[55] Iliade, XVIII, 503-504.

[56] LeFÈVRE-Novaro 2014, 2, p. 227. 


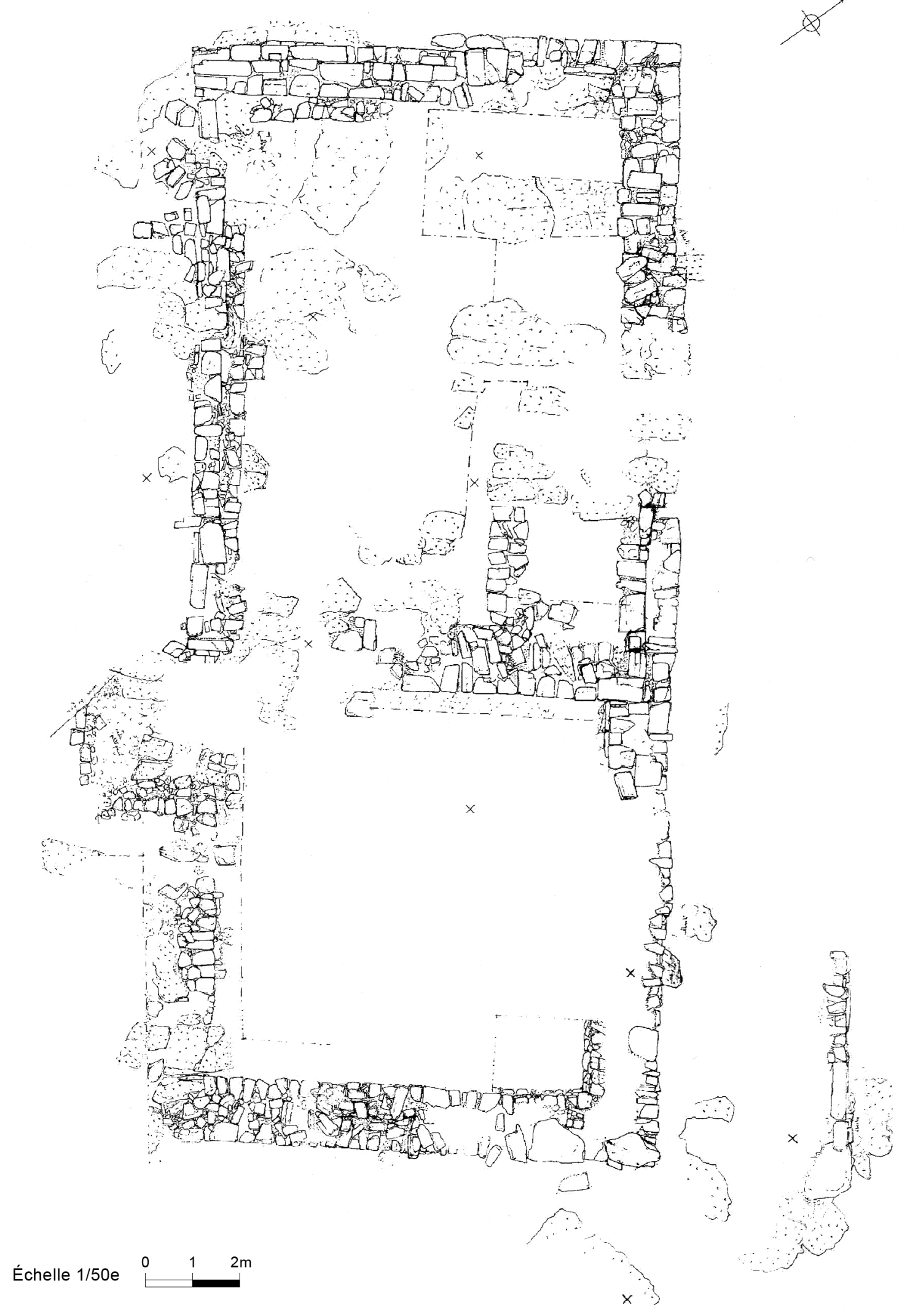

Figure 7

Plan pierre à pierre du temple sur l'acropole ouest

(d'après Zographaki \& Farnoux 2014, p. 107, fig. 5.4). 


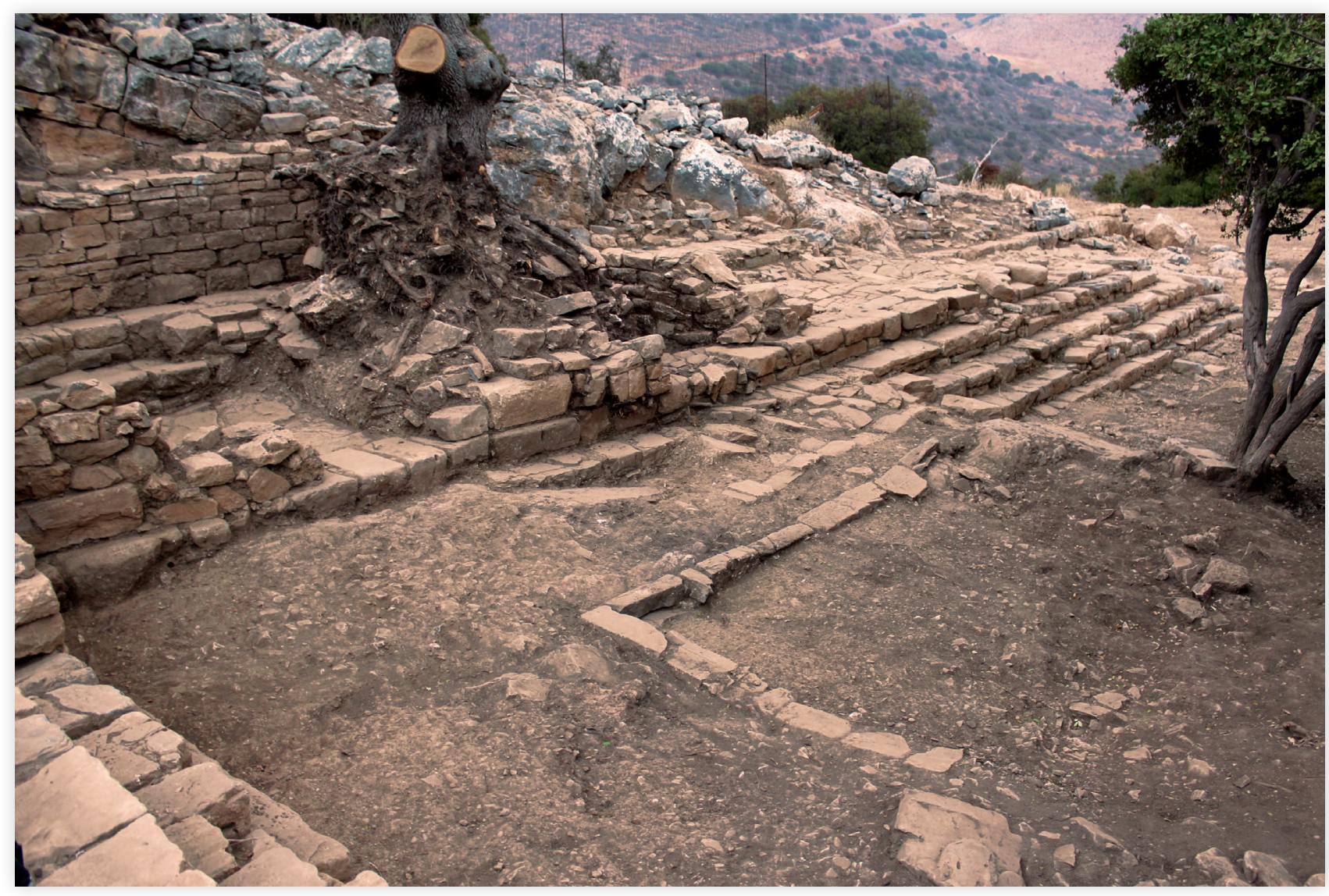

Figure 8

Le secteur occidental de l'agora de Dréros, avec les gradins, la pièce à banquette et l'exèdre. Photo : D. Lefèvre-Novaro.

méridional du plateau. Or, tout en tenant compte du fait que les vestiges pourraient se trouver à un niveau inférieur, cette zone semble correspondre au point de jonction entre les trois orientations repérées jusqu'à présent dans la voirie de la ville. Il faut donc considérer avec attention I'hypothèse émise par F. Longo que l'agora d'époque classique-hellénistique se trouve dans cette zone [57]. Cet emplacement serait en effet plus central que celui d'une agora en contrebas de la colline du palais, du côté sud-est, que nous avions déjà défini comme excentré [58]. Des recherches ultérieures devront venir

[57] LoNGo 2015, p. 169 : en réalité le spécialiste parle plus prudemment de « [...] spazio libero, forse a carattere pubblico ».

[58] LefÈVRE-Novaro 2014, 2, p. 226-227.

[59] CuCuzza 2013, p. 35-37 ; LefÈvre-Novaro 2014, 2, p. 227.

[60] LONGO 2015, p. 166-167.

[61] Pernier 1935, pl. 4 (murs en rouge).

[62] CUCUZZA 2013, p. 35.

[63] Cucuzza \& Ferrari 2004, p. 53-96 pour les kernoi minoens attestés à Phaistos ; CucuzZA 2010 avec bibliographie précédente. étayer cette intéressante hypothèse qui n'exclut pas cependant l'idée que l'ancienne zone du théâtre minoen, à l'ouest du palais, ait été en partie réutilisée lors des phases les plus anciennes de la cité pour des cérémonies communautaires, éventuellement aussi à caractère politique [59]. L'hypothèse de F. Longo [60] selon laquelle les gradins du théâtre auraient été à l'époque hellénistique invisibles, couverts par la pente de la colline, ne peut pas être correctement vérifiée puisque la plupart des structures d'époque historique ont été démontées. Des éléments intéressants sur cette question pourraient en revanche venir d'un réexamen attentif de la section publiée par L. Pernier [61], et mentionnée par N. Cucuzza [62], où I'on voit deux murs « helléniques » installés directement sur le gradin supérieur de la zone théâtrale minoenne qui devait donc être visible. On remarque d'ailleurs que toute la partie orientale de ce secteur n'est pas occupée par des édifices d'époque historique. S'agirait-il d'un « vide » lié à une réutilisation publique des vestiges de I'Âge du Bronze, qui seraient donc visibles, comme à Cnossos à la même époque ? N'oublions pas enfin le phénomène de la réapparition des kernoi d'ancienne tradition minoenne [63] 
dans les contextes publics d'époque historique. La fouille récente du secteur ouest de l'agora de Dréros a révélé une cinquième pierre à cupules provenant du site [64] et des kernoi semblables se trouvent à l'intérieur du grand édifice public découvert à Azoria [65].

À propos de l'agora de Dréros, il est clair que les installations découvertes jusqu'à présent au nord du temple d'Apollon remontent à l'époque hellénistique [66]. Mais des sondages profonds au centre de la place et le long des gradins sud ont commencé à révéler des structures plus anciennes : vers le centre de la place, devant les gradins occidentaux (fig. 8), on a découvert une structure dont I'orientation ne correspond pas à celle du complexe monumental d'époque hellénistique. En outre deux constructions semi-circulaires, oblitérées par les gradins méridionaux, semblent correspondre à des puits installés dans un secteur caractérisé par un ruissellement souterrain [67]. Seule la poursuite des fouilles permettra de comprendre la nature et la fonction de ces structures mais leur présence est d'autant plus significative si l'on pense que les déplacements des agorai sont rares dans le monde grec. En revanche on connaît bien la relation très étroite et ancienne qui existe entre le principal temple urbain et la place publique ; il suffit de songer, entre autres, aux exemples d'Érétrie, de Corinthe et de Métaponte. En définitive I'hypothèse selon laquelle I'agora archaïque de Dréros se trouvait à proximité du temple d'Apollon Delphinios, bien que formulée par $\mathrm{P}$. Demargne et $\mathrm{H}$. Van Effenterre d'après des arguments peu probants [68], semble encore la plus vraisemblable.

En ce qui concerne l'agora de Praisos, très peu d'informations sont disponibles, notamment aux débuts de la polis. On remarque cependant que la morphologie de la zone, avec la présence de trois collines disposées en hémicycle (fig. 4), crée une zone protégée à l'ouest et des dépressions entre les acropoles tout à fait adaptées pour accueillir éventuellement des rassemblements. La proximité de la colline de l'autel et de la première acropole, sièges des plus importants sanctuaires de la ville, est un autre élément significatif dont il faut tenir compte, outre l'analogie avec des sites comme Lato et Dréros où les agorai furent installées dans les ensellements entre les collines.

\section{LA CHRONOLOGIE DE LA STRUCTURATION DE LA POLIS}

En ce qui concerne la chronologie de la mise en place de la polis dans les différentes régions de l'île, Dréros et Praisos se développent à partir du $\mathrm{VIII}^{\mathrm{e}}$ siècle, comme la plupart des cités grecques, mais les données concernant le tissu urbain ne sont pas assez nombreuses pour pouvoir analyser dans le détail ce processus. Phaistos (fig. 1), en revanche, présente des indices qui invitent à réfléchir sur la possibilité que la formation de la polis ait débuté au cours de la seconde moitié du IX siècle [69]. En effet les premières traces d'une communauté nombreuse et organisée remontent aux phases PGR - PGB ( $I X^{e}$ siècle) : la colline du palais était à cette époque déjà occupée par un quartier d'habitations doté d'une rampe dallée qui céda la place au PGB à des habitations et fut réaménagée, toujours avec un dallage et des parapets, juste à l'ouest. Un autre quartier existait peut-être déjà à Chalara, où D. Levi a découvert des vases datés du PG, et sans doute sur la colline de Christos Effendi [70]. Plusieurs nécropoles sont attestées à proximité de la colline du palais, près des villages d'Haghios Ioannis (tholos PG avec objets d'importation), de Petroképhali et vraisemblablement au lieu-dit Ambéli [71]. Le sanctuaire extra-urbain d'Haghia Triada recommença à recevoir des ex-voto à partir du PGB et la fortification sur l'Acropole Médiane pourrait remonter à cette époque. Certes, le lieu primitif de réunion des citoyens reste inconnu et les premières attestations de rituels d'initiation des jeunes membres de l'élite de Phaistos dans le sanctuaire urbain remontent aux dernières décennies du VIII ${ }^{\mathrm{e}}$ siècle (boucliers et chaudrons en bronze), mais l'ensemble des données,
[64] Pour la publication de trois pierres à cupules déjà découvertes à Dréros, cf. LefÈvRE-Novaro 2016. La quatrième a été trouvée pendant la prospection de 2013, dans la zone au nord des deux acropoles ; une cinquième est visible sur le seuil de la pièce à banquette mise au jour dans le secteur occidental de l'agora (fig. 8). Je remercie V. Zographaki et A. Farnoux, directeurs de la fouille de Dréros, pour m'avoir permis de les publier.

[65] HAGgIS et alii 2011, p. 19-21, fig. 10 et 15.
[66] ZographaKi \& FARNOUx 2014, p. 111 pour une position prudente sur la chronologie de l'agora.

[67] Ibid., p. 110.

[68] Demargne \& Van Effenterre 1937, p. 11.

[69] Pour le détail des données archéologiques, cf. LefÈVRe-Novaro 2007, p. 477-488 ; LefÈVRE-Novaro 2014 , 2, p. 233, fig. 46.

[70] LoNGO 2015, p. 164.

[71] LefÈvre-Novaro 2014, 2, p. 228-231. 
et notamment le déplacement de la rampe dallée près du quartier géométrique, permettent de supposer que la mise en œuvre du processus de concertation politique à l'intérieur d'une communauté florissante était déjà engagé vers la fin du Ixe siècle [72]. Des indices chronologiques qui vont dans le même sens ont été mis en lumière à Prinias, importante cité crétoise installée au centre de l'île [73] : I'organisation du tissu urbain, et notamment de la partie centrale de la ville, est vraisemblablement à dater du PGB/GA [74]; la consécration du sanctuaire urbain remonte aussi au PGB, comme l'a montré la découverte par A. Pautasso d'un dépôt de fondation sous le sol du temple $A$, construit au cours du VII ${ }^{\mathrm{e}}$ siècle [75].

À Gortyne, la cité rivale de Phaistos en Messara occidentale, les traces de ce développement précoce sont visibles dans la tombe à tholos d'Haghios Georghios [76], appartenant vraisemblablement à un clan qui devait jouer au PGB un rôle économique important (importation de vases de Cnossos, broches en fer d'origine probablement chypriote) ainsi que dans le développement du sanctuaire sur l'acropole dès le PGB [77]. On attend en outre la publication des résultats des fouilles sur la colline de Prophitis Ilias où N. Allegro est en train de découvrir un important quartier d'habitations avec voirie, daté d'époque géométrique-orientalisante, mais fréquenté déjà à partir du PG [78].

\section{CONCLUSIONS}

Ces données concernant Phaistos et d'autres cités de la Crète centrale induisent l'idée d'un développement précoce des poleis crétoises, dans la deuxième moitié du IX ${ }^{e}$ siècle [79]. Elles font écho à
I'hypothèse avancée par plusieurs spécialistes que la composition des poèmes homériques, où la polis est déjà décrite dans le détail, remonte au $I x^{e}$ siècle [80]. Les récentes recherches archéologiques invitent donc à considérer avec une attention accrue le rôle central joué par la Crète dans le développement de la polis, vraisemblablement en raison de ses anciennes traditions en matière d'organisations étatiques et des relations précoces et fécondes avec la Méditerranée orientale que nous avons déjà analysées ailleurs. Il est tout à fait envisageable que les marchands et les artisans levantins qui, après l'avoir longuement fréquentée, s'installèrent dans l'île [81] apportèrent non seulement des influences artistiques, bien attestées dans les boucliers en bronze, I'orfèvrerie et les productions céramiques à partir du PGB, mais aussi de nouvelles idées en matière d'organisation politique. N'oublions pas que les citéÉtats phéniciennes, formes d'organisation politique ayant d'évidentes analogies avec les poleis [82], $s^{\prime}$ étaient déjà structurées au cours des $\mathrm{X}^{\mathrm{e}}-\mathrm{I} \mathrm{x}^{\mathrm{e}}$ siècles tandis que les royaumes chypriotes étaient en train de voir le jour grosso modo à la même époque que les cités grecques [83].

À la différence des Eubéens, peuple qui a joué un rôle actif dès le PG comme vecteurs de marchandises et d'idées entre l'Égée et la Méditerranée orientale [84], les Crétois ont exploité I'emplacement géographique de leur île sans s'en éloigner forcément. Le rôle joué par ces deux peuples fut à l'évidence différent, mais le résultat final fut semblable puisque le nouveau système politique de la polis se développa précocement dans ces deux îles.

Mais les influences proche-orientales ne suffisent pas à expliquer la naissance précoce de la cité-État en Égée. En réalité un autre aspect rapproche la Crète et l'Eubée [85] : elles n'ont été touchées que
[72] LefÈVRe-Novaro 2007, p. 488.

[73] Pour une description complète de cet important site archaïque, cf. LeFÈVRE-NovARO 2014, 2, p. 21-54.

[74] PAUTASSO 2014, p. 62.

[75] PALERMO et alii 2007, p. 272-277, fig. 9-13 (contribution d'A. Pautasso).

[76] LefÈvre-Novaro 2014, 2, p. 181.

[77] LEFÈVRE-NovARo 2014, 2, p. 183-191.

[78] AlLEgro 2015, p. 213.

[79] Pour une chronologie basse voir KOTSONAS 2002.

[80] RuiJgh 1995, p. 96 (IX e siècle) ; CARLIER 1999 (fin IX $^{\mathrm{e}}-\mathrm{VIII}^{\mathrm{e}}$ siècle).

[81] Outre la très controversée tombe J de Tekke (Cnossos), les attestations les plus sûres de Levantins installés en Crète remontent aux VIII ${ }^{\mathrm{e}}$ et $\mathrm{VII}^{\mathrm{e}}$ siècles :
Kommos (SHAW \& SHAW 2000); Knossos (KouROU \& KARETSOU 1998, p. 243-254) ; Eleutherna (STAMPOLIDIS 2004, p. 238).

[82] Ces analogies ont été soulignées à maintes reprises : voir en dernier ELAYI 2013, p. 107-109. La question des analogies et des différences entre les citésÉtats phéniciennes et grecques mériterait d'être reprise à la lumière des nouvelles données archéologiques.

[83] À propos de la naissance du royaume d'Amathonte au Cypro-Geometric III (fin IX - début VIII ${ }^{\mathrm{e}}$ siècle), voir Petit 2015. Pour les dimensions plus importantes des royaumes chypriotes par rapport au territoire des cités crétoises, cf. I'intéressant tableau publié par WHITLEY 2014 , p. 144 , tableau 7.1 .

[84] LEMOS 2002, p. 228-229.

[85] Voir aussi LEMBESI 1996 pour les analogies concernant I'iconographie et les aspects artistiques. 
marginalement pas la baisse démographique et le dépeuplement survenus à la fin du système palatial égéen, au cours du XII siècle. L'Eubée (Lefkandi), comme d'ailleurs I'Attique (Pérati) et la Locride orientale (Mitrou) situées juste de l'autre côté du canal euboïque, loin d'être dépeuplées, ont connu à l'époque un apport de populations dont I'origine doit être mieux précisée. En Crète, les recherches de ces dernières années ont montré que généralement au cours du XII ${ }^{\mathrm{e}}$ siècle les habitants ne se sont déplacés que de quelques kilomètres par rapport aux établissements du MR III, en cherchant souvent refuge sur les hauteurs, mais sans abandonner l'île. Or si I'on tient compte du fait que I'organisation des poleis est probablement le fruit d'une longue évolution dans laquelle ont joué un rôle essentiel d'un côté les influences proche-orientales et de l'autre les réminiscences d'anciennes collectivités administratives locales mycéniennes comme les damoi [86], il est fort significatif de voir que la polis apparaît tout d'abord dans les deux régions égéennes qui, en l'état actuel de nos connaissances, témoignent au mieux de ces différents apports pendant le haut-archaïsme.

En conclusion, les fouilles récentes en Crète semblent indiquer que le processus de concertation politique à l'origine des poleis pourrait avoir débuté dès la seconde moitié du IX ${ }^{e}$ siècle, c'est-à-dire avant l'époque à laquelle on s'accorde généralement à dater les débuts des institutions politiques dans le monde égéen. Ces indices invitent donc à considérer avec une attention accrue I'hypothèse selon laquelle la polis serait en réalité le résultat d'un long processus, dont les origines remontent aux phases finales du système palatial égéen, enrichi ensuite par de nouvelles idées provenant du Proche-Orient et de Chypre, arrivées en Crète et en Eubée avant leur diffusion dans le reste du bassin égéen.

[86] WeRLINGS 2010, p. 21-30.

\section{BIBLIOGRAPHIE}

Allegro, Nunzio, 2015, «Profitis Ilias (Gortina). Nuovi dati e prospettive di ricerca », dans Daniela Lefèvre-Novaro et alii (éd.), Géosciences, archéologie et histoire en Crète de l'Âge du Bronze récent à l'époque archaïque, Actes du Colloque international, Strasbourg 16-18 octobre 2013, Padova, p. 211-219.

Alusik, Tomas, 2007, Defensive Architecture of Prehistoric Crete (BAR International, Series 1637), Oxford.

Anzalone, Rosario Maria, 2015, Gortina VII. Città e territorio dal protogeometrico all'età classica (Monografie della Scuola Archeologica di Atene e delle missioni italiane in Oriente XXII), Atene.

Belli, Paolo, 1991, «Tholoi nell'Egeo dal II al I millennio », dans Domenico Musti et alii (éd.), La transizione dal Miceneo all'alto arcaismo. Dal palazzo alla città, Atti del Convegno Internazionale, Roma 14-19 marzo 1988 (Incunabula Graeca 98), Roma, p. 425-450.

BosanqueT, Robert, 1901-1902, «Excavations at Praesos I », Annual of British School at Athens 8, p. 231-270.

Camberlein, Claire, 2015, « Observations préliminaires sur le rôle des keimelia en Crète du XIV ${ }^{\mathrm{e}}$ au VII ${ }^{\mathrm{e}}$ siècle avant J.-C. », dans Daniela Lefèvre-Novaro et alii (éd.), Géosciences, archéologie et histoire en Crète de l'âge du Bronze récent à l'époque archaïque, Actes du Colloque international, Strasbourg, 16-18 octobre 2013, Padova, p. 319-331.

Carlier, Pierre, 1999, Homère, Paris.

Coutsinas, Nadia, 2013, Défenses crétoises. Fortifications urbaines et défense du territoire en Crète aux époques classiques et hellénistiques (Cahiers archéologiques de Paris $1, n^{\circ} 3$ ), Paris.

CrielaARd, Jan Paul 2009, « Cities », dans Kurt Raaflaub \& Hans Van Wees (éd.), A Companion to Archaic Greece, United Kingdom, p. 349-372.

CucuzzA, Nicola, 2010, « Game Boards or Offering Tables ? Some Remarks on the Minoan 'pierres à cupules' », Kernos 23, p. 133-144.

Cucuzza, Nicola, 2013, « Minoan Ruins in Archaic Crete», dans Wolf-Dietrich Niemeier et alii (éd.), Kreta in der geometrischen und archaischen Zeit, Akten des Internationalen Kolloquiums, Athen, 27.-29. Januar 2006 (Athenaia $n^{\circ} 2$ ), München, p. 31-42. 
Cucuzza, Nicola \& Ferrari, Chiara, 2004, «I cosiddetti kernoi di Festos », Creta Antica 5, p. 53-96.

D'Acunto, Matteo, 2002-2003, « Il tempio di Apollo a Dreros: il culto e la 'cucina del sacrificio' », Annali dell'Istituto Orientale di Napoli (Archeologia) n. s. 8-9, p. 9-62.

D'Acunto, Matteo, 2013, «The city siege and the lion. The Fortetsa bronze belt and quiver between Near Eastern models and heroic ideology », dans Wolf-Dietrich Niemeier et alii (éd.), Kreta in der geometrischen und archaischen Zeit, Akten des Internationalen Kolloquiums, Athen, 27.-29. Januar 2006 (Athenaia n² 2), München, p. 471-487.

Demargne, Pierre \& Van Effenterre Henri, 1937, « Recherches à Dréros », Bulletin de Correspondance Hellénique 61, p. 5-32.

Duнoux, Yves, 1982, L'étéocrétois. Les textes - la langue, Amsterdam.

ElayI, Josette, 2013, Histoire de la Phénicie, Paris.

Gagarin, Michael \& Perlman, Paula, 2016, The laws of ancient Crete c. 650 - 400 BCE, Oxford.

GaIgnerot-Driessen, Florence, 2016, De l'occupation postpalatiale à la cité-État grecque : le cas du Mirambello (Crète) (Aegaeum 40), Leuven - Liège.

GeHRKE Hans-Joachim, 2009, « States», dans Kurt Raaflaub \& Hans Van Wees (éd.), A Companion to Archaic Greece, United Kingdom, p. 395-410.

GuARDUCCI, Margherita, 1967, Epigrafia greca, I, Roma.

HAggrs, Donald, et alii 2011, «Excavations in the Archaic Civic Buildings at Azoria in 2005-2006 », Hesperia 80, p. 1-70.

HANSEN, Mogens Herman 2006, Polis. An Introduction to the Ancient Greek City-State, Oxford.

Hansen, Mogens Herman \& Nielsen, Thomas Heine (éd.), 2004, An Inventory of Archaic and Classical Poleis, Oxford.

JefFerY, Lilian Hamilton 1990, The Local Scripts of Archaic Greece, $2^{\mathrm{e}}$ éd. (1 $1^{\mathrm{re}}$ éd. 1961), Oxford.

Kotsonas, Antonis, 2002, « The Rise of the Polis in Central Crete », Eulimene, 3, p. 37-74.

Kourou, Nota \& KARETSOu Alexandra, 1998, «An Enigmatic Stone from Knossos: a Reused Cippus ? », dans Vasso Karageorghis \& Nicolaos Stampolidis (éd.), Eastern Mediterranean : Cyprus - Dodecanese - Crete 16th-6th Century B.C., Proceedings of the International Symposium, Rethymnon, 13-16 May 1997, Athens, p. 243-254.

LAFON, Xavier et alii, 2011, La ville antique. Histoire de l'Europe urbaine 1, $2^{\mathrm{e}}$ éd. (1' éd. 2003), Paris.

LA Rosa, Vincenzo, 2000, « Venti anni di ricerche a Festos ed Haghia Triada », dans Un ponte fra I'Italia e la Grecia, Atti del Simposio in onore di Antonino Di Vita, Ragusa, 13-15 febbraio 1998, Padova, p. 31-38.

LA Rosa, Vincenzo, 2010, « Phaistos », dans Eric Cline (éd.), The Oxford Handbook of the Bronze Age Aegean (ca. 3000-1000 B.C.), Oxford, p. 582-595.

Lefèvre-Novaro, Daniela, 2007, « Les débuts de la polis (I'exemple de Phaistos - Crète) 》, Ktèma 32, p. 467-495. Lefèvre-Novaro, Daniela, 2009, « Culti e santuari a Festòs in epoca altoarcaica. Per un'analisi funzionale », CretaAntica 10/2, p. 563-597.

Lefèvre-Novaro, Daniela, 2014, Du massif de I'Ida aux pentes du mont Diktè. Peuples, territoires et communautés en Messara du XIII e au VII e siècle av. J.-C., vol. 1-2, Paris.

Lefèvre-Novaro, Daniela, 2016, « Osservazioni preliminari sui cosiddetti kernoi scoperti a Creta in contesti dell'età del Ferro », dans Julie Patrier et alii (éd.), Mille et une empreintes. Un Alsacien en Orient. Mélanges en I'honneur du $65^{e}$ anniversaire de Dominique Beyer (Subartu XXXVI), Turnhout, p. 205-213.

LembesI, Angeliki, 1996, « The Relations of Crete and Euboea in the Tenth and Ninth Centuries BC. The Lefkandi Centaur and his Predecessors », dans Don Evely et alii (éd.), Minotaur and Centaur. Studies in the Archaeology of Crete and Euboea Presented to Mervyn Popham (BAR International, Series 638), Oxford, p. 146-154.

Lemos, Irene, 2002, The Protogeometric Aegean. The Archaeology of the Late Eleventh and Tenth Centuries BC, Oxford. LeVI, Doro, 1956, « Attività della Scuola Archeologica Italiana di Atene nell'anno 1955 », Bollettino d'Arte 41, p. 238-274.

LoNGo, Fausto, 2015, «Considerazioni preliminari sulla topografia della città greca di Festos », dans Daniela LefèvreNovaro et alii (éd.), Géosciences, archéologie et histoire en Crète de l'âge du Bronze récent à l'époque archaïque, Actes du Colloque international, Strasbourg, 16-18 octobre 2013, Padova, p. 159-181.

Marinatos, Spiridon, 1936, « Le temple géométrique de Dréros I-II », Bulletin de Correspondance Hellénique 60, p. 214-285.

Matthaüs, Hartmut, 2011, «The Idaean Cave of Zeus: the Most Important Pan-Cretan Sanctuary. Evidence of Metalwork», dans Giovanni Rizza (éd.), Identità culturale, etnicità, processi di trasformazione a Creta fra Dark Age e Arcaismo. Per i cento anni dello scavo di Prinias, 1906-2006, Atti del convegno, Atene, 9-12 novembre 2006, Catania, p. 109-132.

Minto, Antonio, 1921-1922, «Fortificazioni elleniche di Festos », Annuario della Scuola Archeologica Italiana di Atene 4-5, p. 161-175.

MoNTECCHI, Barbara, 2007, « Alcune riflessioni sugli AN $\triangle P E I A$ e sulle $A \Gamma E \wedge A I$

cretesi », Annuario della Scuola Archeologica Italiana di Atene 85, p. 83-117.

NowICKI, Krzysztof, 2000, Defensible Sites in Crete C. 1200 - 800 B.C. (LM IIIB/IIIC Through Early Geometric) (Aegaeum 21), Liège - Austin.

Palermo, Dario et alii 2007, «Lo scavo del 2005 sulla Patela di Priniàs. Relazione preliminare », Creta Antica 8, p. 265-314.

Pautasso, Antonella, 2014, «Prinias. Une cité crétoise de l'Âge du Fer et son histoire », dans Florence GaignerotDriessen \& Jan Driessen (éd.), Cretan Cities : Formation and Transformation (Aegis 7), Louvain-La-Neuve, p. 59-77.

Perlman, Paula, 2004, «Writing on the Walls. The Architectural Context of Archaic Cretan Laws », dans Leslie Preston 
Day et alii (éd.), Crete beyond the Palaces, Proceedings of the Crete 2000 Conference (INSTAP Prehistory Monographs, $\mathrm{n}^{\circ}$ 10), Philadelphia, p. 181-197.

Pernier, Luigi, 1935, Il palazzo minoico di Festos. Scavi e studi della missione archeologica italiana a Creta dal 1900 al 1934. Vol. I. Gli strati più antichi e il primo palazzo, Roma.

Petrt, Thierry, 2015, «La ville et le royaume d'Amathonte n'ont pas été fondés au XI siècle », dans Daniela LefèvreNovaro et alii (éd.), Géosciences, archéologie et histoire en Crète de l'âge du Bronze récent à l'époque archaïque, Actes du Colloque international, Strasbourg, 16-18 octobre 2013, Padova, p. 353-375.

Polignac, François de, 1991, La nascita della città greca. Culti, spazio e società nei secoli viII e VII a. C., (traduction italienne mise au jour ; $1^{\text {re }}$ éd. française 1984), Milano.

Prent, Mieke, 2005, Cretan Sanctuaries and Cults. Continuity and Change from late Minoan III C to the Archaic Period, Leiden - Boston.

Rolley, Claude, 1994, La sculpture grecque 1. Des origines au milieu du ve siècle, Paris.

RuIJGH, Cornelis Jord, 1995, « D'Homère aux origines protomycéniennes de la tradition épique », dans Jan Paul Crielaard (éd.), Homeric Questions. Essays in Philology, Ancient History and Archaeology, Amsterdam, p. 1-96.

Shaw, Joseph \& Shaw, Maria (éd.) 2000, Kommos IV. The Greek Sanctuary, 1, Princeton.

Snodgrass, Antony, 1993, «The Rise of the Polis. The Archaeological Evidence », dans Mogens Herman Hansen (éd.), The Ancient Greek City-State, Symposium at the Royal Danish Academy of Sciences and Letters, July, 1-4 1992 (Acts of the Copenhagen Polis Centre, $\mathrm{n}^{\circ} 1$ ), København, p. 30-40.

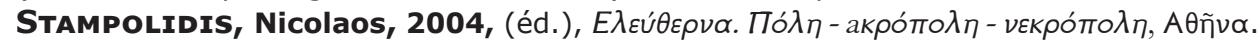

TSIPOPOULOU, Metaxia, 2005, $H$ Anatoliki Kriti stin proimi epoki tou Siderou, Héraklion.

VAN EffenterRe, Henri, 2009, La nécropole de Dréros (Études crétoises 8/2), Atene - Napoli.

VAN Effenterre, Henri \& RuzÉ, Françoise, 1994-1995, Nomima I-II. Recueil d'inscriptions politiques et juridiques de l'archaïsme grec (Collection de l'École française de Rome, n 188), Roma.

Wallace, Saro, 2010, Ancient Crete. From Successful Collapse to Democracy's Alternatives, Twelfth to Fifth Centuries B.C., Cambridge.

Werlings, Marie-Josephine, 2010, Le dèmos avant la démocratie. Mots, concepts, réalités historiques, Paris.

WhItLeY, James, 1998, «From Minoans to Eteocretans: the Praisos Region 1200-500 B.C. », dans William Cavanagh \& Michael Curtis (éd.), Post-Minoan Crete, Proceedings of the First Colloquium at the University College, London 10th11th November 1995 (Annual of British School at Athens, Studies 2), Nottingham, p. 27-39.

Whrtley, James, 2006, « Praisos : Political Evolution and Ethnic Identity in Eastern Crete C. 1400-300 BC », dans Sigrid Deger-Jalkotzy \& Irene Lemos (éd.), Ancient Greece: from the Mycenaean Palaces to the Age of Homer, Edinburgh, p. 597-617.

WhrteeY, James, 2014, «Commensality and the "Citizen State" », dans Florence Gaignerot-Driessen \& Jan Driessen (éd.), Cretan Cities : Formation and Transformation (Aegis 7), Louvain-La-Neuve, p. 141-163.

WhItLey, James, et alii, 1995, « Praisos III : a Report on the Architectural Survey Undertaken in 1992 », Annual of British School at Athens 90, p. 405-428.

Whitley, James, et alii, 1999, « Praisos IV : a Preliminary Report on the 1993 and 1994 Survey Seasons », Annual of British School at Athens 94, p. 215-264.

ZographaKI, Vasso \& FARnOUX, Alexandre, 2014, «Dréros : cité et sanctuaires », dans Florence GaignerotDriessen \& Jan Driessen (éd.), Cretan Cities : Formation and Transformation (Aegis 7), Louvain-La-Neuve, p. 103-117. 


\section{ENSILAGE ET ARTISANAT DU FER \\ ENTRE LE HALLSTATT D3 ET LA TĖNE ANCIENNE \\ À WEYERSHEIM (BAS-RHIN). \\ PREMIERS RÉSULTATS}

Matthieu MICHLER

Chargé d'études et d'opérations

\section{Sylvain BADEY}

Technicien d'opération - Archéomaticien, Inrap, UMR7324 CITERES Laboratoire Archéologie et Territoires sylvain.badey@inrap.fr

\section{Marion BERRANGER}

Ingénieure de recherche CNRS - Archéométallurgiste, UMR 5060 IRAMAT Belfort

marion.berranger@utbm.fr

\section{Luisella CABBOÏ}

Chargé d'études et d'opérations, Inrap, UMR 5060 IRAMAT Belfort

luisella.cabboi@inrap.fr

\section{Patrick CLERC}

Responsable d'opération, Inrap, UMR 5060 IRAMAT Belfort patrick.clerc@inrap.fr

\section{Florent JODRY}

Responsable d'opération, macrolithicien, Inrap, UMR 7044 Archimède

florent.jodry@inrap.fr

\section{Olivier PUTELAT}

Archéo-zoologue, Archéologie Alsace, UMR 7041 ArScAn « Archéologies Environnementales » Université de Paris 1 Panthéon-Sorbonne / MAE Nanterre

olivier.putelat@archeologie.alsace

\section{Marieke van ES}

Céramologue, Inrap

marieke.van-es@inrap.fr 


\section{RÉSUMÉ}

Le site de Weyersheim «Les Hauts de la Zorn » (Bas-Rhin), fouillé en 2014 par I'Inrap a livré pour la première fois en Alsace les restes d'un atelier de forge, sur un site occupé de la fin du Hallstatt au début de La Tène ancienne.

Outre la mise au jour de nombreux silos, ayant livré des artefacts céramiques et métalliques typiques, deux structures (silo 1003 et fosse 1014) situées au sud-ouest de l'emprise, se singularisent par la quantité impressionnante de déchets métallurgiques découverte (110 kg de scories incluant près de 280 culots). L'analyse de ces déchets a mis en évidence un travail de post-réduction, depuis l'épuration du métal brut à la mise en forme d'objet.

Des outils lithiques, ainsi

\section{Mots-CLÉs}

Âge du Fer,

habitat,

Alsace,

production,

fer,

forge,

enclumes,

outils lithiques, scories. qu'un important lot d'enclumes en granite ont également été mis au jour.

Cette découverte atypique permet de nous interroger sur la place de la métallurgie en Alsace au ve siècle av. J.-C.
The site of Weyersheim «Les Hauts de la Zorn » (Bas-Rhin), excavated in 2014 by the Inrap, delivered for the first time in Alsace the remains of a forging workshop on a site occupied at the end of Hallstatt period and the beginning of La Tène period.

In addition to the discovery of numerous silos, having delivered typical ceramic and metallic artifacts, two structures (silo 1003 and pit 1014) localized at the southwest are distinguished by the impressive amount of metallurgical wastes $(110 \mathrm{~kg}$ of smithing wastes including almost 280 smithing slags). The analysis of the metallurgical waste revealed a post-reduction work, from bloomsmithing to the shaping of objects. Lithic tools, as well as a large batch of granite anvils, were also found. This atypical discovery allows us to question the place of metallurgy in Alsace in the 5th century BC.

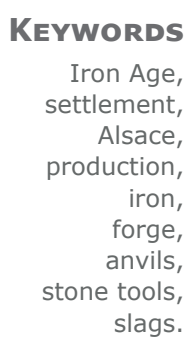


Le site de Weyersheim «Les Hauts de la Zorn » est localisé dans la plaine rhénane, à $15 \mathrm{~km}$ au nord de Strasbourg (fig. 1). Il occupe une zone assez plane (142,4 m NGF en moyenne) située à I'ouest du ban communal, rue de la Gare. Un léger pendage orienté N/S est visible, en direction du lit de la rivière Zorn. Sa découverte est consécutive aux recherches archéologiques liées à la réalisation d'un futur lotissement (6,2 ha diagnostiqués) [1]. La zone prescrite par le Service régional de I'Archéologie, d'une surface de 9900 m2, concerne le quart nord-ouest du projet (fig. 2) [2].

Bien que la majeure partie des vestiges mis au jour soient datables entre le Hallstatt D3 et La Tène ancienne, notons la découverte d'un dépôt de crémation du Bronze final I-IIb (str. 1060 [3]) à l'est, six structures oblongues non datées s'apparentant aux fosses à profils en $\mathrm{V}$ ou $\mathrm{Y}$ connues au Néolithique et à la Protohistoire [4] et deux structures modernes et contemporaines (une petite structure de chauffe et un silo à betterave).

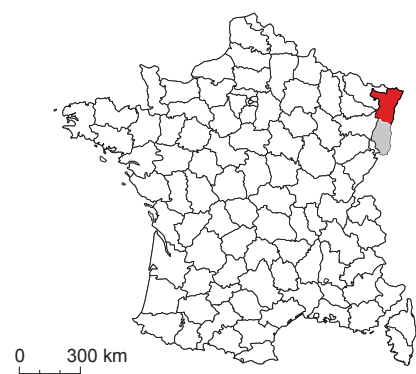

Figure 1 : localisation du site de Weyersheim dans le Bas-Rhin et des autres sites contemporains ayant livré des traces d'artisanat du fer (points rouges sur la carte). DAO : M. Michler.

1 - Rosheim
2 - Bischoffsheim
3 - Wolfisheim
4 - Holtzheim
5- Entzheim
6 - Souffelweyersheim
7 - Duntzenheim
8 - Gougenheim
9 - Mittelhausen
10 - Mittelshaeffolsheim

Les excavations de l'âge du Fer se répartissent quasiment sur l'ensemble de l'emprise de fouille (excepté à l'extrême est) et le site semble se développer au-delà de la limite ouest où se trouvent les structures liées au travail du fer. Un petit bâtiment sur poteau a été repéré au nord et la distribution spatiale des silos montre des effets d'alignement et de contrainte (deux bandes au nord et au sud). À proximité de ces structures de stockage se distingue la fosse 1014, où se pratiquait une activité de forge (accumulation de battitures et de restes de foyers en place). C'est la structure la plus originale du site, avec le silo 1003 qui a servi de fosse de rejet pour des déchets scorifiés. Lors du diagnostic, des déchets avaient également été

\section{[1] DABECK 2013.}

[2] MICHLER 2016.

[3] Datation sur os : 2925+/- 30BP, soit 1257-1019 BC à deux sigmas (Poz-71716).

[4] ACHARD-COROMPT \& RIQUiER 2013.

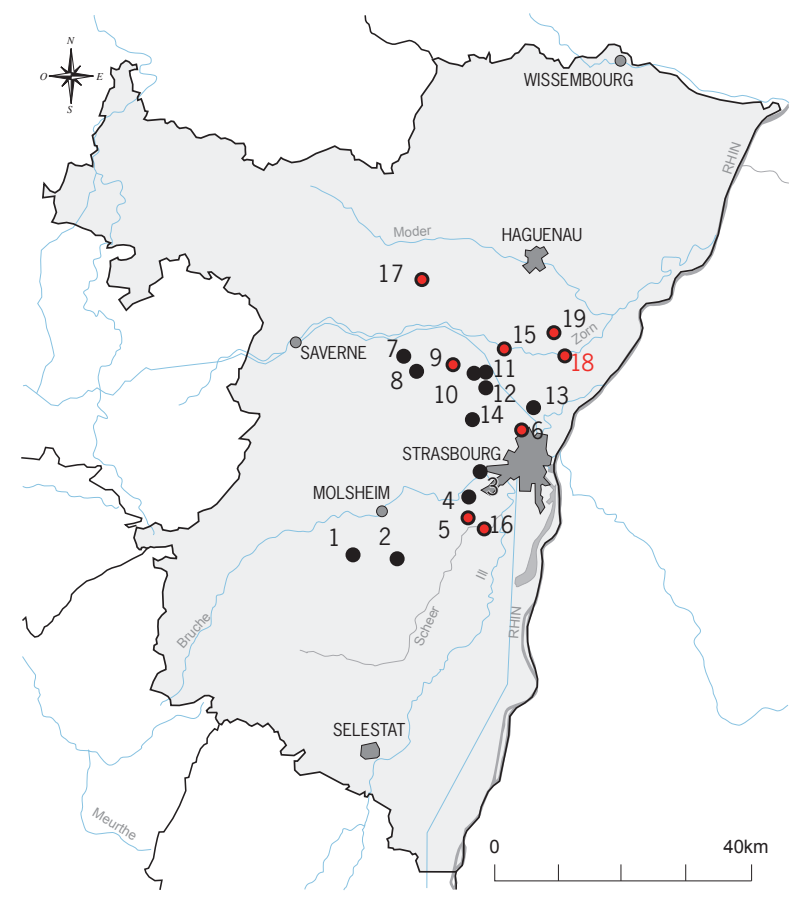




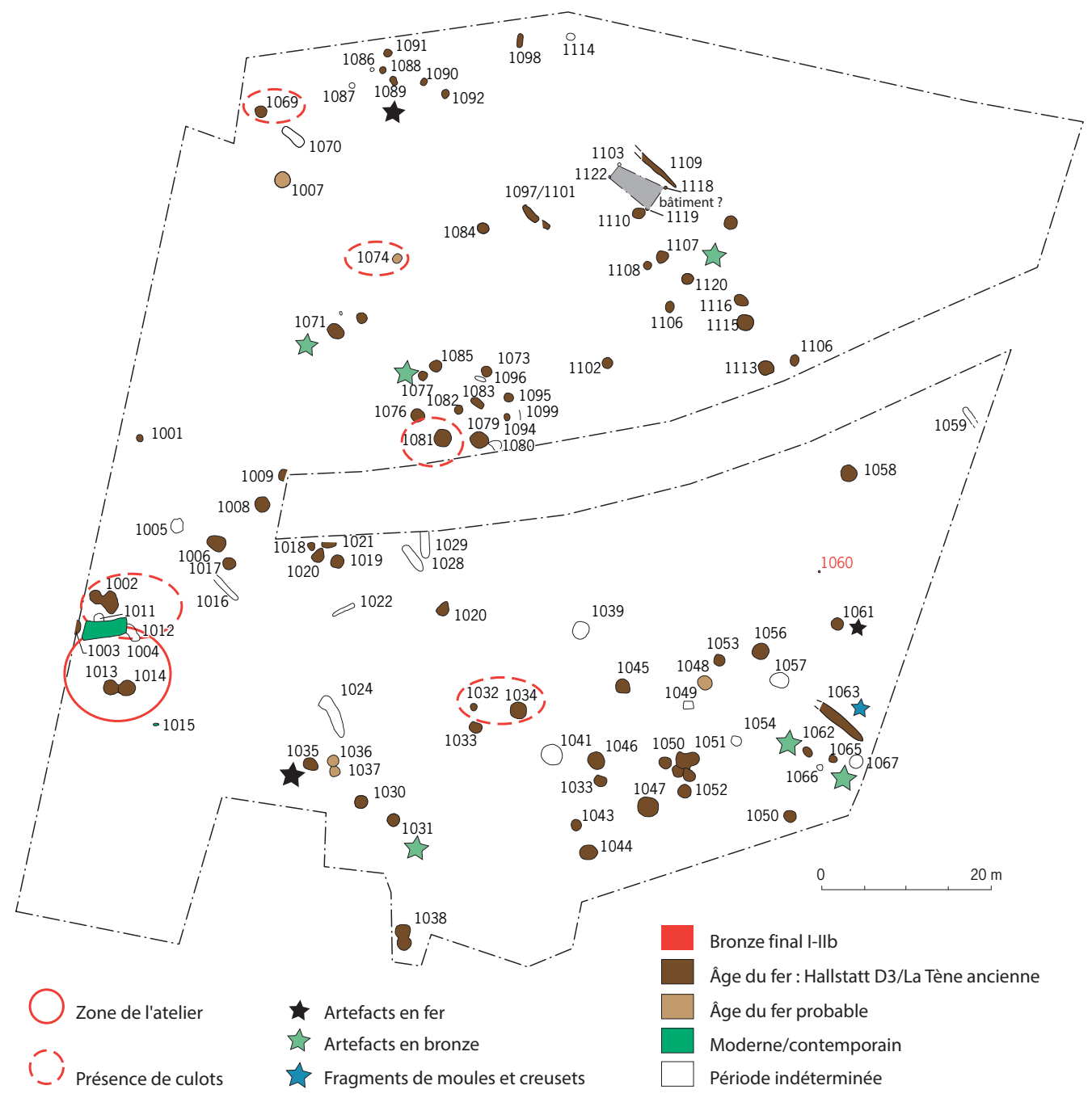

Figure 2

Plan phasé du site avec les structures ayant livré des objets en fer ou en alliage cuivreux, ainsi que des restes liés à l'artisanat du métal.

DAO : M. Michler, J.-L. Wuttmann, P. Girard.

découverts dans certaines structures (str. 19, 32, 40 et 45), mais les traces d'un artisanat du métal étaient illustrées par une seule fosse de combustion de taille réduite et mal datée (str. 07, plus à l'est, secteur non prescrit). De tels vestiges d'activité métallurgique encore inconnus régionalement ont par conséquent fait l'objet d'analyses poussées permettant d'illustrer une vocation particulière du site, même s'ils s'intègrent dans un contexte d'ensilage bien identifié, comme c'est le cas pour d'autres sites alsaciens contemporains.

Alors qu'en contexte rural les sites contemporains de Weyersheim ne livrent généralement que des indices d'une activité métallurgique ponctuelle, celui de« Les Hauts de la Zorn » se caractérise par une grande quantité de déchets liés à un travail de forge. Une place plus importante sera donc réservée à la présentation des activités métallurgiques. L'analyse que nous proposons complète d'une

[5] GentNer 2016. certaine façon l'article sur l'économie du fer dans le nord de la Forêt-Noire présentée dans cette même revue [5] en 2016.

\section{L'OCCUPATION DU HALLSTATT D3 À LA TĖNE ANCIENNE}

Soixante-douze faits appartiennent à cette phase et recouvrent l'ensemble de la zone prescrite (fig. 2). Des concentrations au nord, au sud et à l'ouest dans la zone de l'atelier suggèrent que le site s'étend par delà l'emprise de la fouille. Une spécificité du lieu est de concentrer à la fois des activités artisanales de forge et des silos.

Outre les structures de stockage (50 exemplaires), des fosses à contours ovales ou irréguliers (une dizaine), des cuvettes (5 exemplaires) et des restes de fossé ( 3 exemplaires) ont été mis au jour. Les artefacts découverts soulignent I'homogénéité chronologique du site. Le corpus céramique est comparable en volume à d'autres sites d'ensilage 


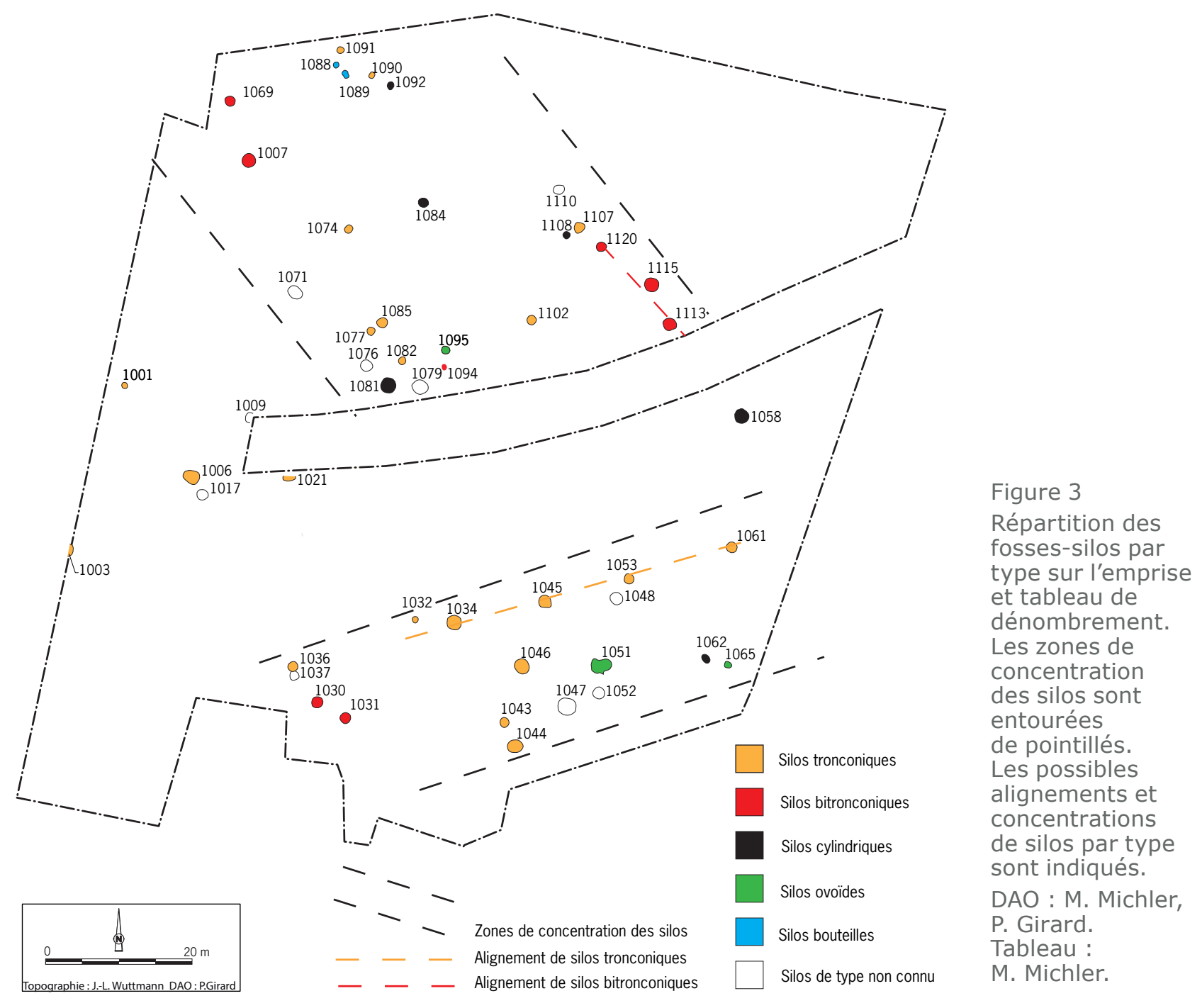

(Mittelhausen, « Liesbuehl/Gimbretter Weg 》 [6] par exemple) et les objets en bronze et en fer sont des parures, de l'outillage ou de l'armement.

\section{ORGANISATION SPATIALE ET NATURE DU SITE}

Les cinquante silos (fig. 3 et $\mathbf{4}$ ), majoritairement de section circulaire ou ovale se répartissent en cinq groupes morphologiques d'après leur profil : tronconiques (fig. 4, 1061), bitronconiques, cylindriques (fig. 4, 1058), ovoïdes (fig. 4, 1065) et en bouteille (fig. 4, 1088). Vingt-cinq ont $0,50 \mathrm{~m}$ ou plus de profondeur et d'après le calcul du taux d'érosion (formule de F. Gransar [7]) seule une vingtaine peut être considérée comme bien conservée. Comme le souligne M. Roth-Zehner, les sites d'ensilage restent

[6] CROUTSCH et alii 2015.

[7] GRANSAR 2002.

[8] MALRAIn et alii. 2013, p. 38.

\begin{tabular}{|c|c|c|c|}
\hline PROFIL & $\mathrm{Nb}$ & $\%$ & Numéros \\
\hline Tronconique & 21 & 42 & $\begin{array}{l}1001,1003,1006,1021,1032,1034,1036,1043 \\
1044,1045,1046,1053,1061,1074,1077,1082, \\
1085,1090,1091,1102,1107\end{array}$ \\
\hline Bitronconique & 8 & 16 & $1007,1030,1031,1069,1094$ B, 1113, 1115, 1120 \\
\hline Cylindrique & 6 & 12 & $1058,1062,1081,1084,1092,1108$ \\
\hline Ovoïde & 3 & & $1051 \mathrm{~A}, 1065,1095$ \\
\hline Bouteille & 2 & 4 & 1088,1089 \\
\hline Non connu & 10 & 20 & $1009,1017,1037,1047,1052,1071,1076,1079,1110$ \\
\hline Total & 50 & 100 & \\
\hline
\end{tabular}

majoritaires en Alsace au premier âge du Fer, et ce jusqu'à La Tène B1 [8]. La prédominance des profils tronconiques est pour le moment une constance sur des sites contemporains.

Deux zones d'ensilage (partie nord et sud de l'emprise) sont visibles avec des largeurs de respectivement 46 et $23 \mathrm{~m}$. La zone de vide relatif entre les deux n'a pas pu être entièrement explorée ce qui limite la réflexion, mais soulignons que l'absence de vestiges pourrait-être liée à une zone de passage ou à la volonté de regrouper les silos pour des raisons pratiques ou topographiques (silos appartenant à différents groupes 


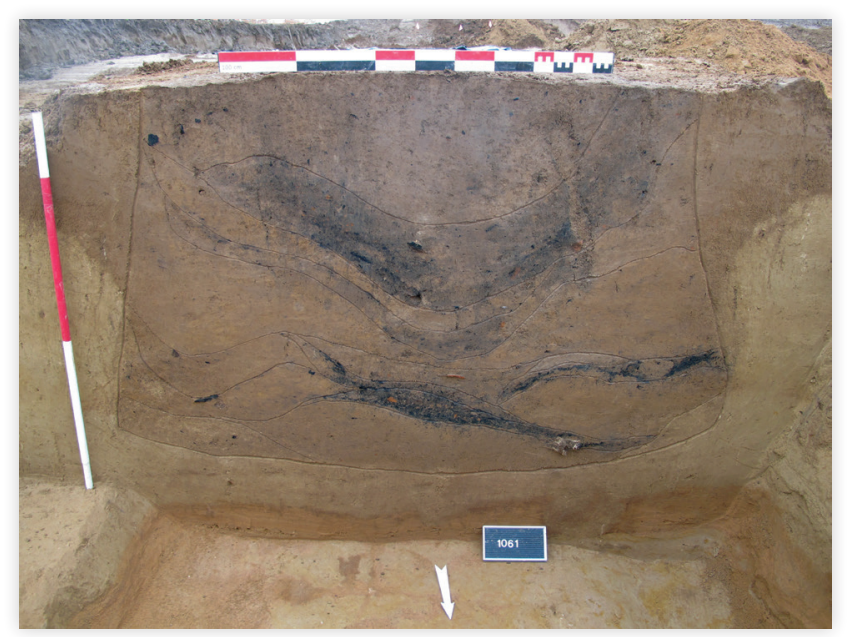

Structure 1061

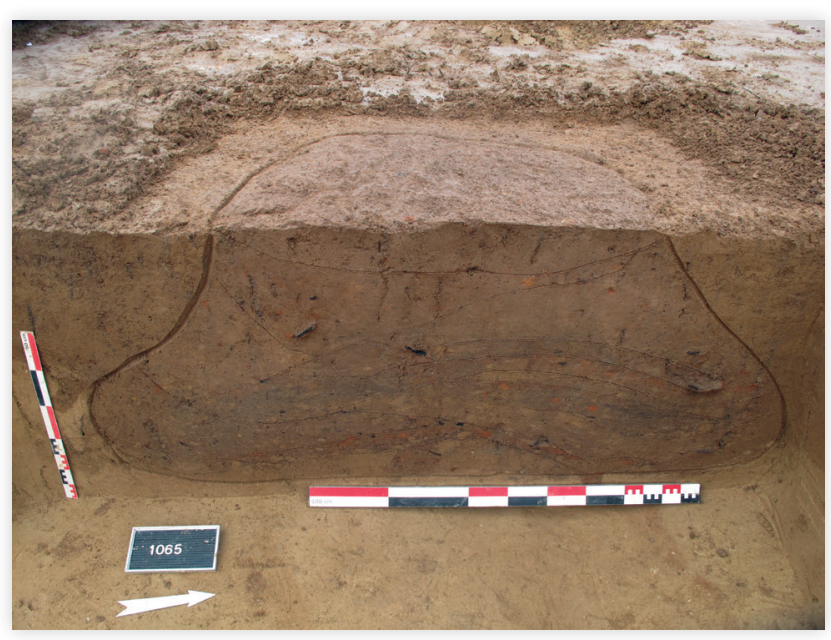

Structure 1065

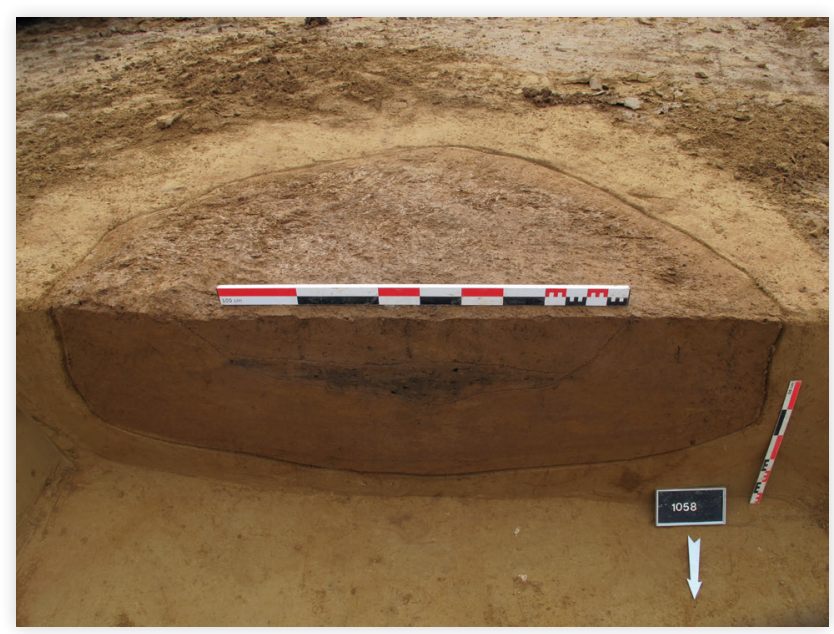

Structure 1058

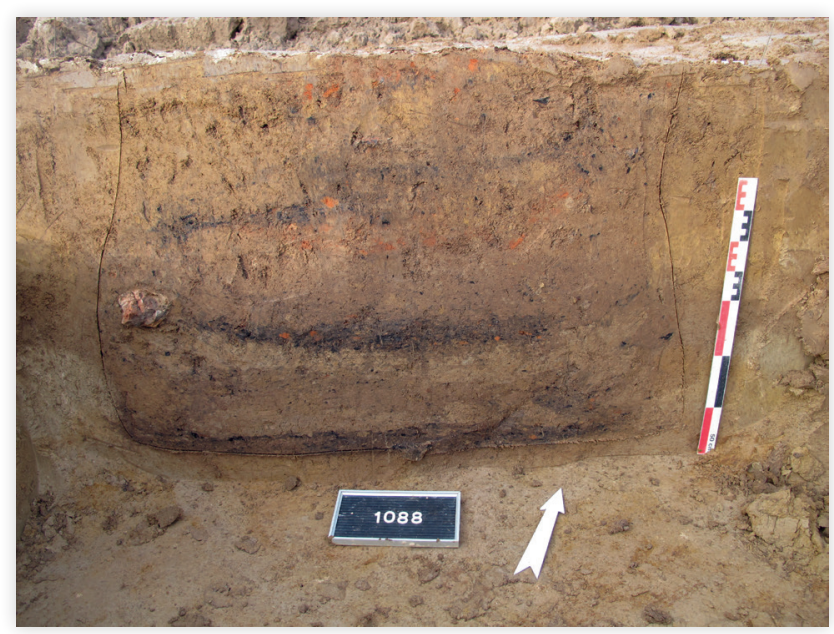

Structure 1088

Figure 4

Fosses-silos représentatives par type. Fosse tronconique (St. 1061), fosse cylindrique (St. 1058), fosse ovoïde (St. 1088), fosse en bouteille (St. 1088).

(C) Dessins et photos, équipe Inrap. DAO : P. Girard, mise en forme M. Michler.

familiaux par exemple, contrôle plus aisé sur des surfaces bien délimitées, réelles contraintes du terrain). Deux alignements de silos (fig. 3) dans la zone sud et au nord sont visibles. Ils pourraient s'agir de silos fonctionnant en batterie comme à Duntzenheim « Schlittweg », «Weingartenberg» [9] (Véber 2015). Le mobilier datant, qui ne permet pas une précision à la génération (environ 25 ans) limite les réflexions sur sujet.

Une tentative de calcul du volume utile des silos conservés à partir de leurs dimensions restituées n'a pas encore été faite. S. Defressigne et $M$. Landolt ont fait ce travail pour d'autres sites alsaciens et lorrains (du Hallstatt B à La Tène B) [10]. Ainsi, La capacité moyenne de stockage par site connaît une progression relativement constante avec le temps avec des capacités exponentielles jusqu'à
La Tène B. Si I'on compare le nombre de ces silos à celui d'autres sites contemporains, Weyersheim est bien doté (cinquante silos) [11], mais pour aller plus loin il faudrait lier ces nombres aux surfaces décapées. Par exemple, par rapport au site de Mittelhausen « Liesbuehl, Gimbretter Weg »[12] où $5000 \mathrm{~m}^{2}$ ont été décapés et trente-six silos mis au jour, la proportion de silo à Weyersheim est plus faible même si le nombre reste un des plus élevés pour la période.

Les limites de l'emprise rendent également difficile la compréhension de la topographie de l'atelier

[9] VÉBER 2015.

[10] Defressigne \& LANDOLT, 2017.

[11] Ibid., fig. 11.

[12] CROUTSCH et alii 2012. 
par rapport aux deux concentrations de silos mentionnés. Le silo 1003 d'où proviennent des culots est à la limite de décapage et pourrait être en lien avec des concentrations situées plus à l'ouest. Quoiqu'il en soit à Weyersheim l'activité métallurgique semble coexister avec les emplacements destinés au stockage (avec toute la prudence liée à la chronologie large), les silos étant très proches (moins de dix mètres). Ceci n'est pas le cas sur d'autres sites d'habitat à vocation agricole où ont été repérées des activités de réduction et de forge. Ainsi sur le site de Gondreville «Au Loup », c'est autour du regroupement de 131 silos que sont installés les grands bâtiments d'habitation et les nombreuses petites constructions de type « grenier surélevé »[13]. Les différentes activités sont bien séparées spatialement (à une centaine de mètres).

Les mêmes questionnements peuvent être posés quant à la position d'un petit bâtiment sur poteaux (peut-être de type «grange », comme à Illfurth «Buergelen » [14]) situé au nord de l'emprise, tout contre des silos et à plus de 70 mètres de l'atelier (figure 2 en grisé). Soulignons que les deux datations $\mathrm{C}^{14}$ sur charbon provenant de deux poteaux tombent dans le plateau du Hallstatt. S'agit-il d'un lieu de vie des populations responsables de ces structures de stockage, d'une zone artisanale de forgerons ou des deux ? Aucune réponse claire ne se dégage pour le moment. En Alsace, les sites datés du Hallstatt D3 à LaTène ancienne regroupant une unité d'habitation, des greniers et des silos sont encore rares, contrairement à la Lorraine où cette association est assez bien connue comme sur le site de Fontenoy-sur-Moselle « Les Herbues de Gondreville »[15]. Cette situation alsacienne résulte également de problèmes taphonomiques pour les structures faiblement ancrées.

Une réflexion plus large sur le statut particulier du site au vu de la présence exceptionnelle d'un atelier de forge et de nombreux rejets métallurgiques sera envisagée ci-après.

\section{PRODUCTIONS ET ARTEFACTS}

\section{La céramique}

Parmi le mobilier archéologique mis au jour, la céramique provient d'une quarantaine de structures et oriente la datation au HaD3/LTA. 2762 tessons ont été identifiés, correspondant à 275 vases pour un poids total de $6 \mathrm{~kg}$. Il s'agit de 145 formes basses pour 60 vases de forme haute. Les céramiques fines et mi-fines constituent le plus gros du corpus avec 173 individus pour 91 vases en céramique grossière. Les silos 1077 (fig. 5) et 1089 ont livré les plus gros ensembles. Les formes basses ouvertes à profil sinueux sont nombreuses. Quelques tessons présentent des dépôts blancs internes. On retrouve aussi des poignées de couvercle ou bien de couvre-feu.

Le «profil type» de la céramique de Weyersheim avec des décors incisés, des petites bouteilles et des formes sinueuses se retrouve au sein de la structure 1229, attribuée au Hallstatt D3, à EntzheimGeispolsheim «Aéroparc (LIDL-CUS)» [16]. Mais des comparaisons avec la céramique des structures 1608 et 1609 d'Entzheim plus tardives (Ha D3-LT A) sont possibles.

Il est important de souligner que la céramique tournée manque dans le corpus. Sa présence peut parfois illustrer un site de rang supérieur, ce qui aurait été envisageable avec la présence de l'atelier de forge. De plus aucun véritable stigmate en lien avec l'activité métallurgique n'a été observé sur les tessons. Toutefois la présence de céramique en situation de rejet, suggère fortement que I'habitat des populations agricoles et des artisans est à proximité.

Des objets assez fréquents pour cette période comme un anneau, une cuillère et six fusaïoles en terre cuite ont également été découverts.

\section{La faune}

177 des 298 restes osseux d'animaux terrestres $(5,2 \mathrm{~kg})$ mis au jour dans la fouille sont datés de la fin du Hallstatt au début de La Tène ancienne (fig. 6). Ils proviennent de 19 des 82 structures excavées attribuées à cette période. Bien que le nombre de structures soit sensiblement égal entre les moitiés nord et sud de l'emprise (fig. 8), seuls deux échantillons (1102 et 1113) appartiennent à la moitié nord, les 17 autres relevant plutôt de la moitié sud. Ce matériel osseux, assez bien conservé, se compose d'un squelette de chien, de cinq ramures de cerfs élaphe et de 124 restes attribués à des déchets alimentaires [17].

Le squelette d'un jeune chien adulte d'environ 24 mois, quasiment complet au moment de sa découverte, a été mis au jour dans le silo 1052 (fig. 8). La posture du cadavre, et sa localisation dans

[13] Defressigne \& LeROY 2013.

[14] Roth-ZEHNER, 2013, p. 59, fig. 4, type 3.

[15] Defressigne \& LANDOLT, 2017, p. 85, fig. 6.

[16] LANDOLT et alii 2007.

[17] PUTELAT 2016. 
Figure 5 : vases issus de la structure 1077.

(C) M. Van Es, P. Girard, Inrap.

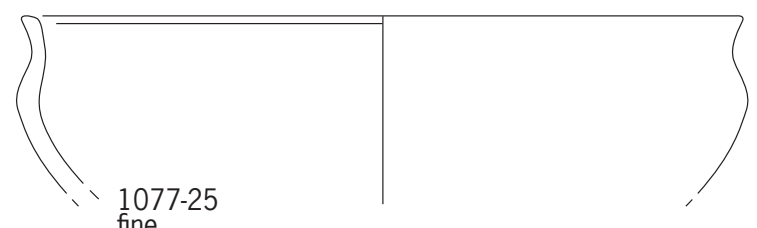

1.
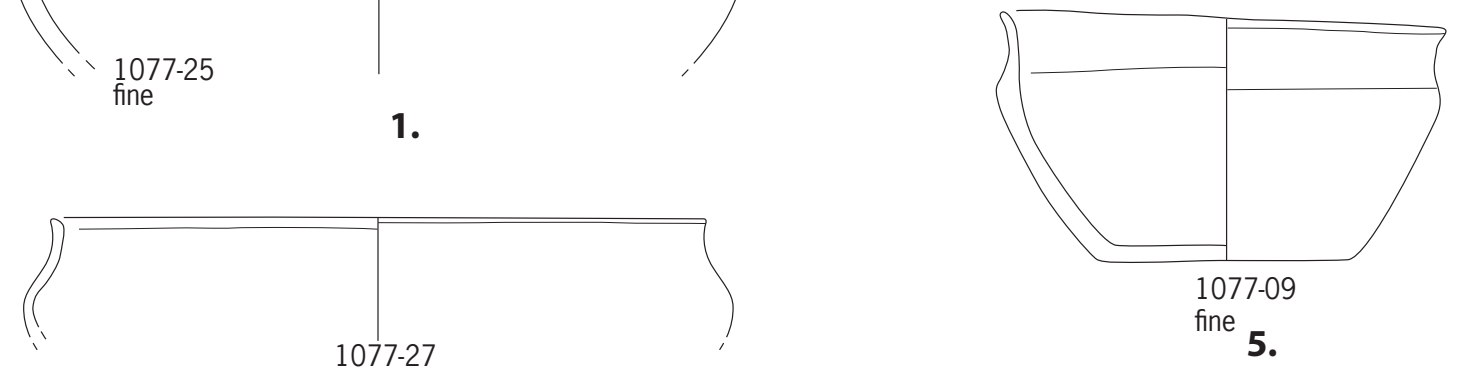

2.

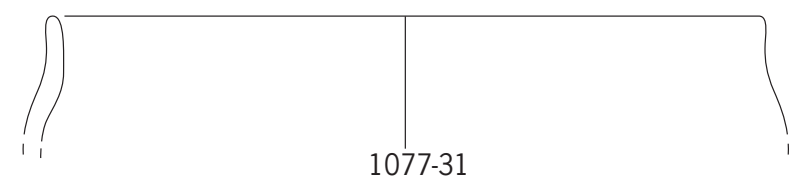

3.

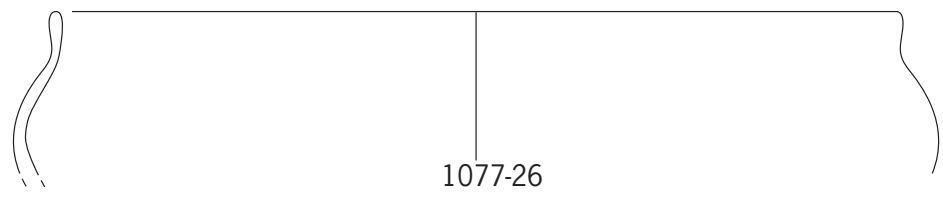

4.

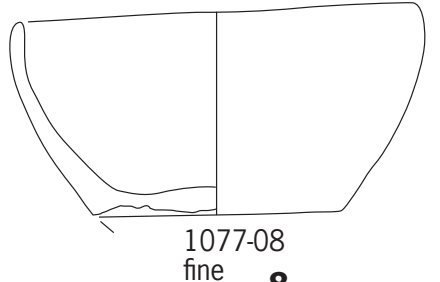

8.

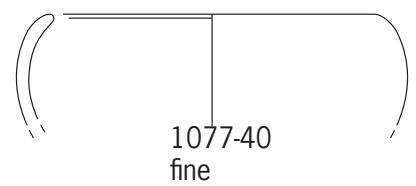

11.

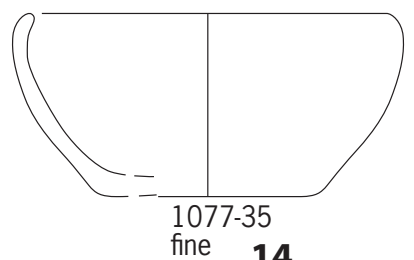

fine 14.

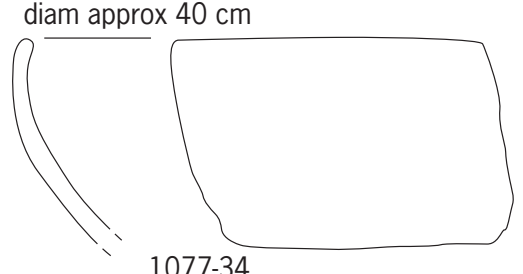

9.

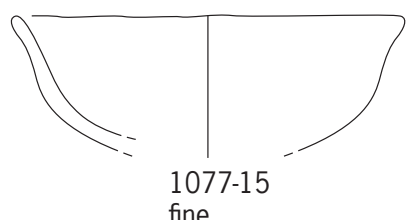

6.

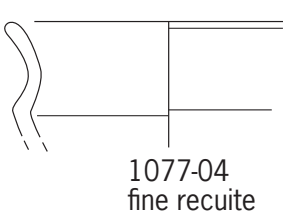

7.

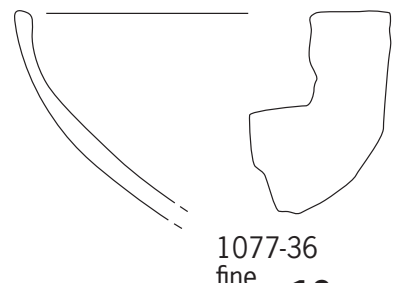

10.

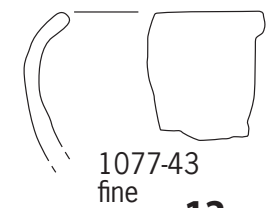

12.

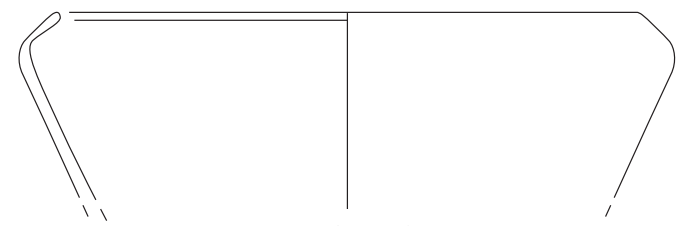

1077-13

13.

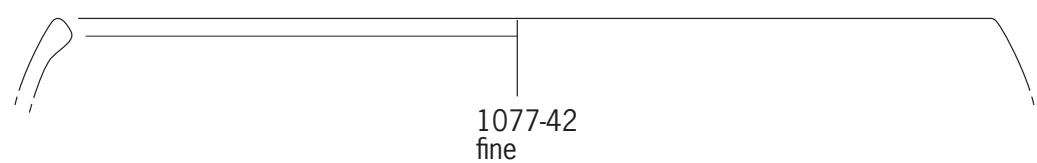

15.

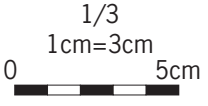


Figure 6

A. Répartition spatiale des ramures de cerfs élaphe et des déchets osseux d'origine culinaire (carrés rouges).

B. Vue d'ensemble des deux ramures du silo 1030.

C. Squelette de chien 1052 in situ.

(c) Photos équipe Inrap, Dao : P. Girard, mise en forme O. Putelat.
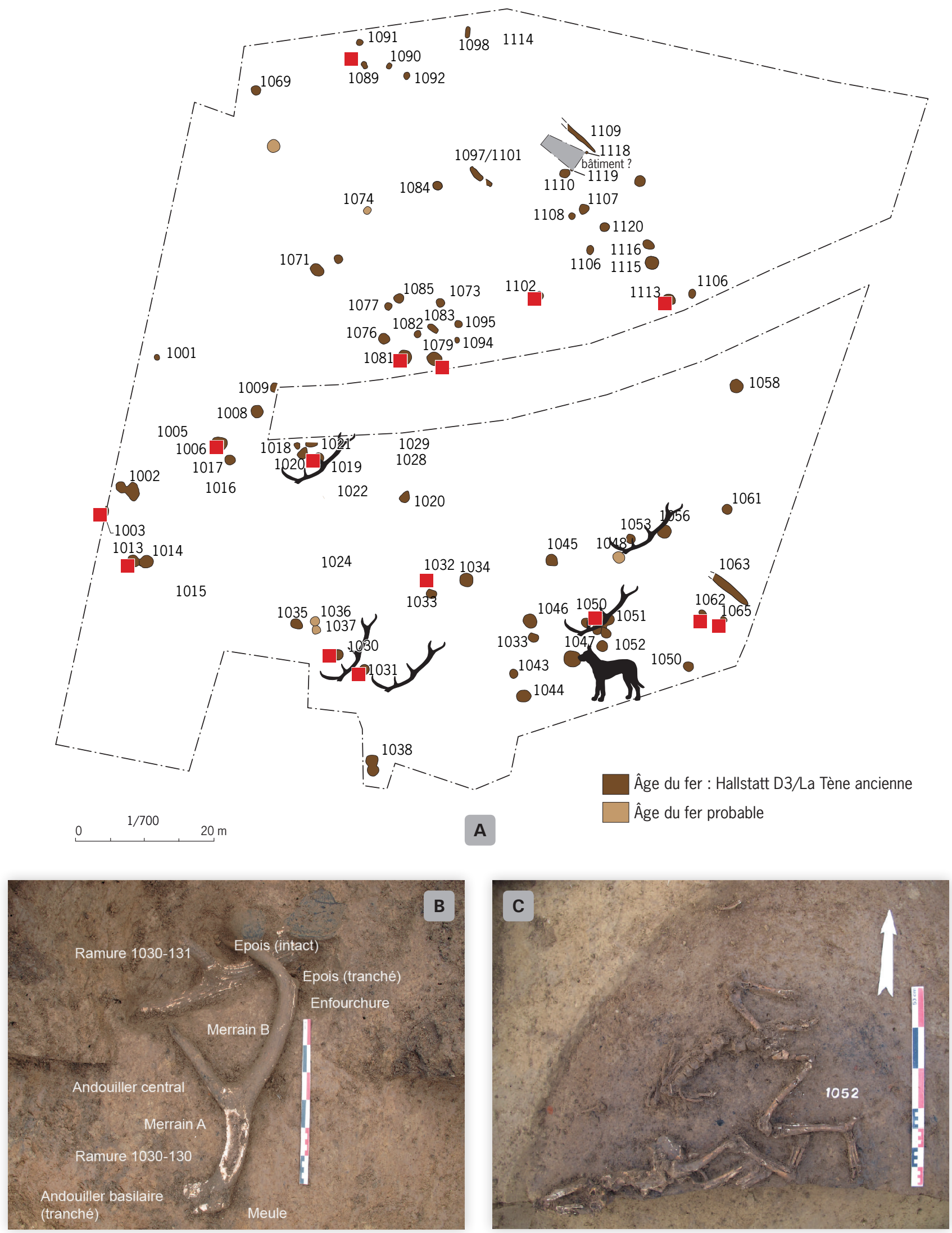

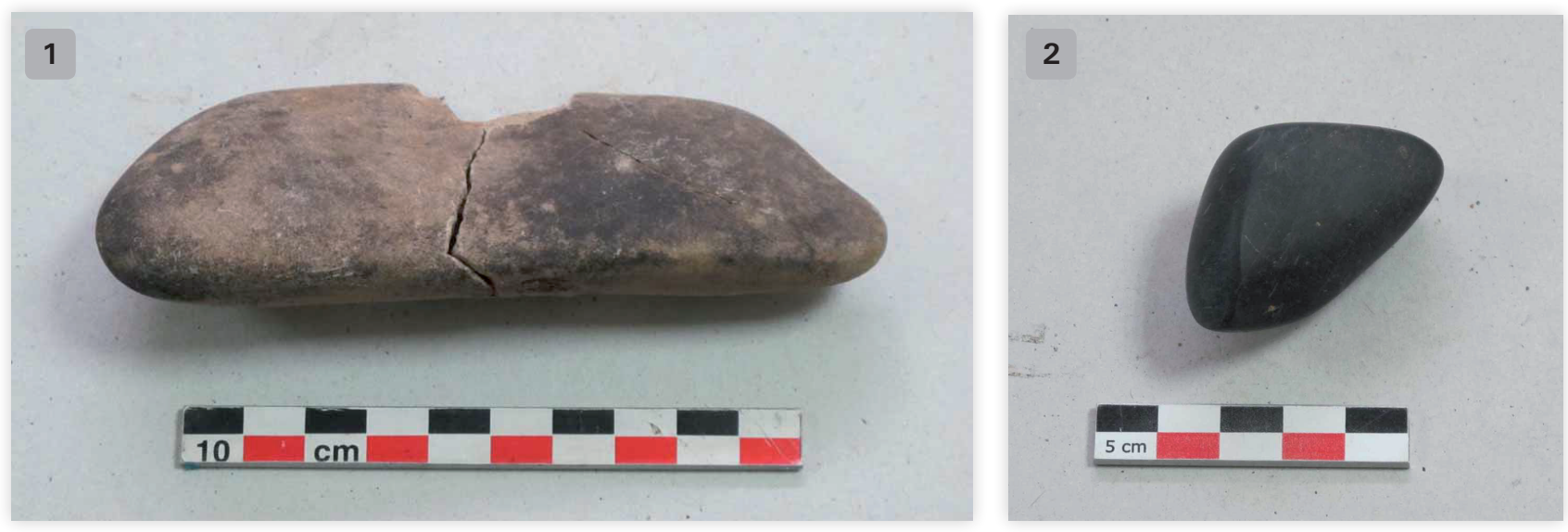

Figure 7 : outillage lithique :

1. Lissoir aiguisoir $1063-03$; 2. Brunissoir ou pierre de touche 1085-2.

(C) F. Jodry, Inrap.

une couche de déblais, évoquent un rejet opportuniste. Toutefois, le rapprochement de cette découverte avec de nombreux autres dépôts de cadavres d'animaux, recensés en Alsace pour La Tène ancienne, invite à ne pas négliger I'hypothèse d'un dépôt intentionnel, différencié de la seule problématique du rejet [18]. Il serait de fait envisageable que le cadavre du chien de Weyersheim s'insère dans l'ensemble des dépôts d'animaux dits « dépôts particuliers » témoignant de « pratiques rituelles domestiques » [19].

Il pourrait en être de même des cinq ramures de cerfs élaphes (dont au moins trois sont des bois de mues), découvertes lors de la fouille des fosses et silos 1020, 1030, 1050, 1053. Leur répartition montre une localisation dans la moitié sud de la fouille (fig. 6). Des prélèvements de matière dure sont avérés dans trois cas sur cinq [20]. Ils restent cependant limités à une partie des pointes, sans concerner les merrains. Il faut de plus noter qu'aucun déchet de débitage de bois de cerf n'a été découvert, ce qui attesterait une activité tant soit peu conséquente et précise du travail des matières dures animales. Ces découvertes de bois peuvent être rapprochées de deux autres trouvailles bas-rhinoises, faites à Entzheim-Geispolsheim «Aéroparc » (fosse 1088, transition Hallstatt D3 La Tène) [21] et Holtzheim «Am Schuesselberg » (fosse 51, Hallstatt D3) [22] pour lesquelles I'hypothèse de dépôts symboliques avait déjà été évoquée.

Les 124 restes osseux attribués à des déchets alimentaires $(1,8 \mathrm{~kg})$ proviennent de 15 faits des 82 faits datés de la séquence de l'âge du Fer et 96 $\%$ d'entre eux ont été mis au jour dans la moitié sud de l'emprise. Les effectifs de chaque taxon sont inférieurs au seuil de représentativité de 50 restes [23], ce qui nous invite à considérer avec prudence ces données qui traduisent surtout des tendances. Le porc (NR : 25 ; NMI : 3) compose le tiers des 76 restes déterminés et prévaut en fréquence d'occurrence, étant présent dans 12 des 15 échantillons. La masse de ces restes ne représente que $14 \%$ du total, mais les parties les plus riches de la carcasse (jambonneaux, jambons) étant les mieux représentées, il est évident que la contribution du porc à la part carnée de l'alimentation est très conséquente, d'autant que la consommation d'animaux lourds semble privilégiée : un juvénile-subadulte d'environ 24 mois et un adulte de réforme, âgé de 5-7 ans contre un infantile-juvénile de 3-12 mois.

Le bœuf (NR : 22 ; NMI : 2) prévaut selon la masse des restes (61 \% du total). Il est présent dans 11 des 15 échantillons, ce qui atteste une bonne répartition spatiale. L'ensemble de ces paramètres converge pour montrer la prévalence de la viande bovine dans la ration carnée.

Les caprinés (NR : 13 ; NMI : 2), où les ovins et les caprins ne sont pas différenciés, occupent la troisième place, en nombre de restes, en nombre d'individus et en fréquence d'occurrence. Cette dernière est de moitié inférieure à celle du bœuf et du porc (6 échantillons sur 15), ce qui tendrait à indiquer une répartition spatiale moins prononcée et donc sans doute une consommation moins courante.

[18] Putelat \& LANDOLT, 2013.

[19] AuXIETTE et alii 2010.

[20] Putelat, 2016.

[21] PUTELAT 2007.

[22] Kuhnle \& Tesnier-Hermetey 2005.

[23] PUTELAT 2015. 
Une pratique cynophagique occasionnelle est attestée par la présence de 11 restes (fosse 1061), marqués de traces de découpe, et attribués à un même jeune chien adulte d'environ 24 mois. Aux équidés ne sont attribués que deux restes. Trois restes (un fragment de tête osseuse de cerf élaphe, une côte et un tibia de sanglier) illustrent la chasse de grand gibier, et, bien que la proportion totale des restes de gibier soit relativement basse (environ 4 $\%$ du NRD), la part non négligeable tenue par la masse des restes de ce grand gibier dans l'échantillon ( $7 \%$, soit autant que les caprinés), laisse entrevoir une certaine importance de l'apport de la chasse à la ration carnée.

Sous l'angle de l'activité métallurgique, il est intéressant de rappeler que la plupart des ossements d'animaux sont issus de la moitié sud de l'emprise, où se situent les silos 1003 et 1014, caractérisés par l'abondance des rejets liés au travail du fer. Il paraît donc probable que ces rejets osseux soient au moins en partie imputables aux forgerons ou à leurs proches. Toutefois, le silo 1003 ne livre que sept restes d'animaux, tandis que le silo 1014 n'en livre apparemment pas. Seuls quatre des 124 restes sont marqués de traces d'ustion non culinaire, ce qui permet d'écarter toute hypothèse d'utilisation délibérée de l'os en tant que combustible durant le travail du métal, à la différence de ce qui avait été observé à La Tène ancienne au nord du Massif jurassien, sur le site de Chevenez « Combe En Vaillard » [24].

La comparaison des données archéozoologiques de Weyersheim avec d'autres, livrées par 14 sites alsaciens (ou géographiquement proches), tous datés entre le Hallstatt final et La Tène ancienne, a été effectuée avec prudence, en raison de la ténuité du corpus faunique de ce site [25]. Parmi les huit principaux taxons (NR8), l'échantillon de Weyersheim se distingue par une proportion de restes de caprinés très inférieure à la moyenne régionale ( $17,1 \%$ versus $36,2 \%$ ) et par une proportion de restes de porcs nettement supérieure à la moyenne régionale (32,9\% versus $21,9 \%)$. La proportion des ossements de grands gibiers, un peu plus élevée que la moyenne régionale (3,9\% versus 1,9\%), doit aussi être soulignée.

La situation géographique de Weyersheim, entre Kochersberg et Ried, ainsi que les caractéristiques environnementales qui en résultent, ont sans doute entraîné ici une plus forte représentation du bœuf et du porc, mieux armés que les caprinés pour s'adapter aux sols humides. La bonne représentation du porc (souvent engraissé en forêt aux périodes anciennes), la mise en évidence de la chasse de grand gibier, et celle de la collecte de bois de mues, ainsi que les relations « homme-forêt » que tout cela implique, doivent sans doute être évaluées en tenant compte du rôle tenu par la métallurgie dans l'existence des occupants du site. Enfin, s'il nous paraît probable qu'une pratique cynégétique plus intense qu'ailleurs est induite par la proximité du Ried et par un certain opportunisme, il n'en reste pas moins que la chasse au sanglier nécessite courage, armement, voire une certaine organisation sociale.

\section{Outillage macro-lithique (hors artisanat lié au métal)}

Sept petits galets de quartzite, de gneiss et de grès fin ont été inventoriés dans le lot des outils lithiques (St. 1006, 1053, 1063, fig. 7). Leurs surfaces actives sont planes ou convexes, d'aspect poli et marquées par quelques impacts et traces noires de frottement. Il s'agit principalement de lissoirs pour la céramique (panse et décors, Leuvrey 1999). La sphère alimentaire est représentée par une seule molette en grès grossier (1063-1) à surface active lisse et polie.

\section{Les objets en alliage cuivreux et en fer (fig. 8)}

Deux bracelets fins en bronze proviennent de structures adjacentes (str. 1071, 1077), comme des éléments de fibules de type F3B1, proches du fossé 1063 d'où proviennent aussi des restes de creusets ou moules en terre cuite - on peut avancer I'hypothèse d'un petit atelier de bronzier dans cette zone, fig. 8, 6 et 7 - et du silo 1061. Un petit galet noir (1085-2, fig. 7, 2), dont la surface active lisse est marquée de microstries et de traces cuivrées pourrait être une pierre de touche, mais c'est à confirmer.

La présence concomitante d'un artisanat du bronze et du fer est connue sur différents sites de cette période et n'est pas limitée aux seuls habitats fortifiés de hauteur (comme «La Cité d'Afrique » à Messein en Lorraine ou le Britzgyberg à Illfurth en Alsace).

Les objets en fer quant à eux se trouvaient dans des fosses et silos éparpillés sur le site. L'herminette (silo 1089) était associée à un lot important de céramique (fig. 8, 2). On a également découvert un fer de lance à flamme large (fig. $8, \mathbf{3}$ ), ainsi qu'un petit ciseau (fig. 8, 1).

[24] Putelat 2010.

[25] Putelat 2016. 


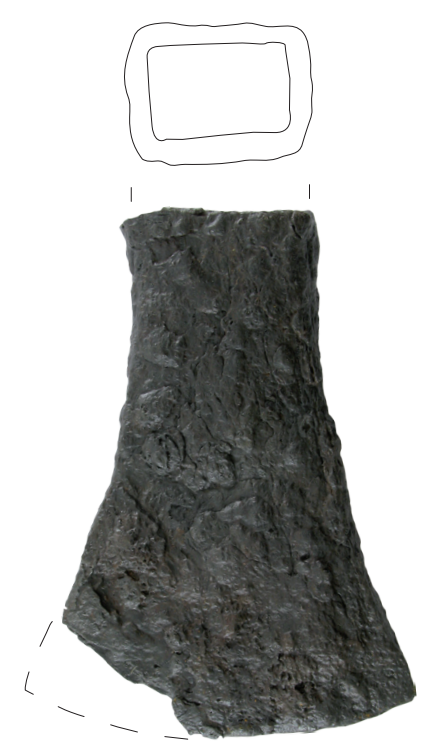

6259-MFE-1089-1

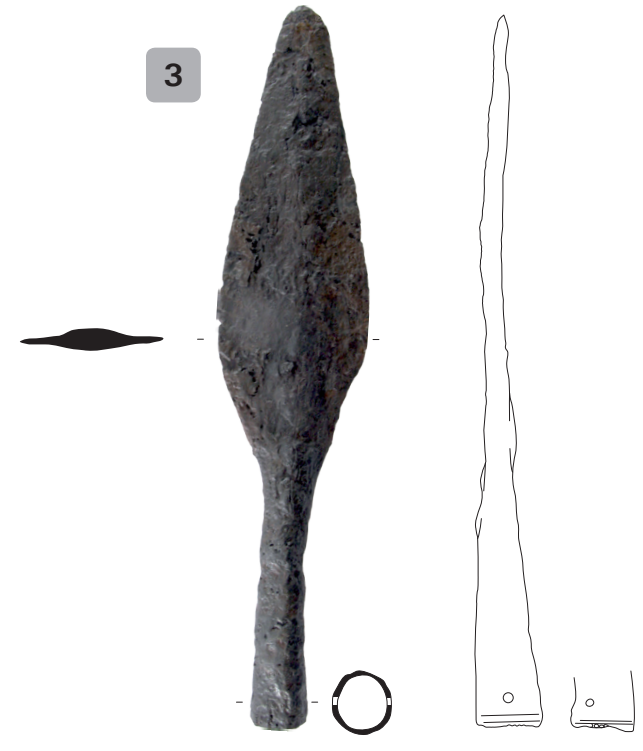

6259-MFE-1061-1

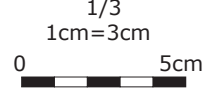

4

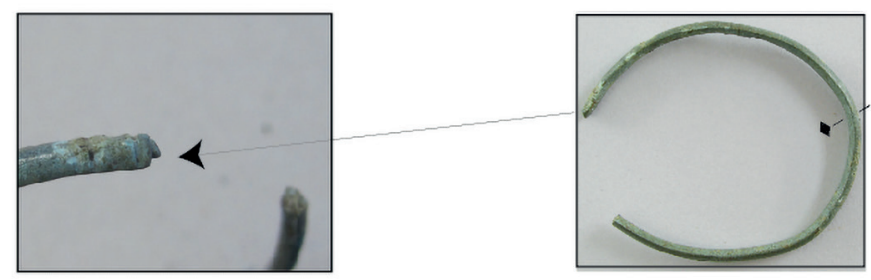

6259-MCU-1071-1
2

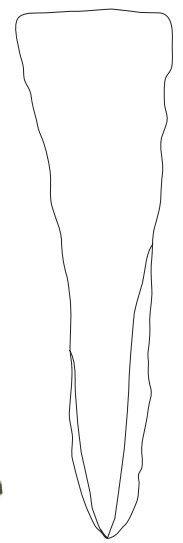

5

Figure 8

Objets en fer :

1. Ciseau, 2. Herminette, 3. Fer de lance.

Objets en alliage cuivreux :

4. Bracelet fin, 5. Fibule à pied décoré,

6 et 7. Creusets et moule en terre cuite.

(C) M. Michler, D. Minni, Inrap.

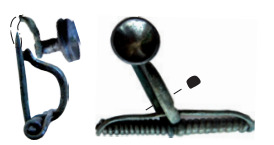

6259-MCU-1062-1 


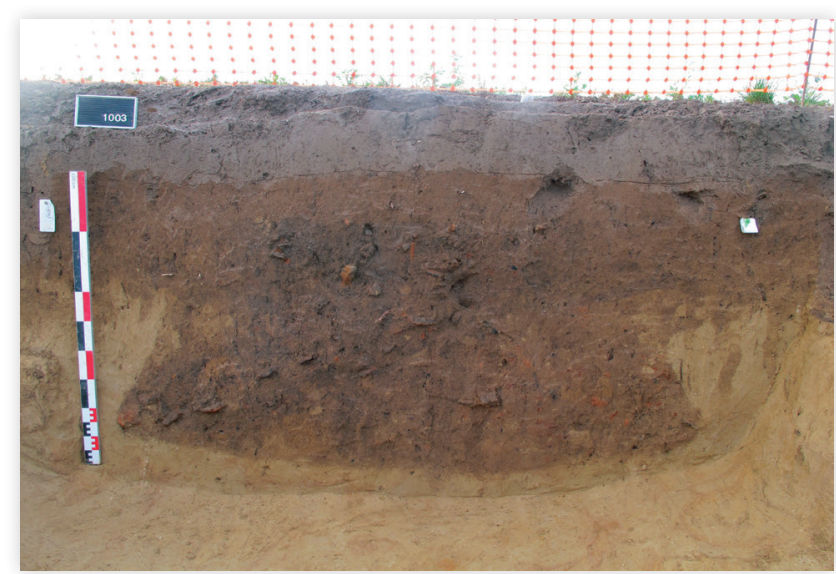

Figure 9 : vue vers l'ouest du silo St.1003, utilisé entre autres comme dépotoir de l'atelier de forge. Seule la moitié est a été fouillée, l'autre moitié est située hors emprise.

(C) cliché P. Clerc, Inrap.

Ces différents objets appartiennent à des types bien connus sur les sites contemporains régionaux et dans le domaine hallstattien centre-occidental [26] (Dubreucq 2013). Pour le moment il n'est pas possible de savoir s'ils proviennent de l'atelier de forge proche, mais leur présence montre que la chasse a pu être pratiquée sur le site (cf. partie sur la faune) tout comme l'artisanat du cuir.

Pour l'artisanat du fer, les déchets métallurgiques se trouvent dans des silos parfois à plus de $70 \mathrm{~m}$ de l'atelier, mais en grande majorité dans la partie ouest de l'emprise. Ces rejets semblent pour le moment aléatoires, comparés à celui massif de nombreux culots dans le silo 1003.

[26] DuBREucQ 2013.

\section{L'ARTISANAT DU FER ET LES STRUCTURES ASSOCIÉES}

Le silo 1003 et la fosse 1014, distante de quelques mètres, concentrent les vestiges d'un atelier de forge abandonné il y a près de 2500 ans.

\section{LE SILO 1003 (FIG. 9)}

Circulaire (diam. 1,40 m) et tronconique, ce silo n'est conservé que sur $1 \mathrm{~m}$ d'épaisseur. En limite d'emprise, seule une moitié a été fouillée et a livré plus de $23 \mathrm{~kg}$ de scories et plusieurs boules de terre cuite. La céramique (formes basses et moyennes à profil sinueux, pots) date le comblement du Ha D3-LTA.

\section{LA FOSSE 1014 (FIG، 10 ET 11)}

Cette fosse semble fonctionner avec une structure voisine très arasée (St 1013) et a livré un matériel archéo-métallurgique exceptionnel, tant par la quantité que par la diversité des éléments.

Trois phases distinctes ont été mises en évidence :

- une phase de fonctionnement s'achevant par la destruction des foyers et le rejet des déchets d'activité sidérurgique.

- une phase de comblement des matériaux issus du démantèlement du (ou des) foyer(s) et d'une bonne partie de l'outillage de l'atelier (enclumes).

- une phase d'abandon et de recouvrement de la fosse.

Les datations $\mathrm{C} 14$ réalisées sur des charbons de bois des niveaux de comblement et de fonctionnement proposent respectivement $2430 \pm 30 \mathrm{BP}$ et $2465 \pm 30 \mathrm{BP}$ (problème du plateau hallstattien entre le viri ${ }^{e}$ et le ve siècle av. J.-C., soit 740-403 et 760-414 BC à deux sigmas).

Figure 10 : vue du début de la fouille de la fosse 1014.

À gauche : mise au jour des premiers grands fragments de terre cuite et des blocs de granite.

À droite : dégagement des niveaux superficiels (US.4, US.12 et US.13).

(C) clichés P. Clerc, Inrap.
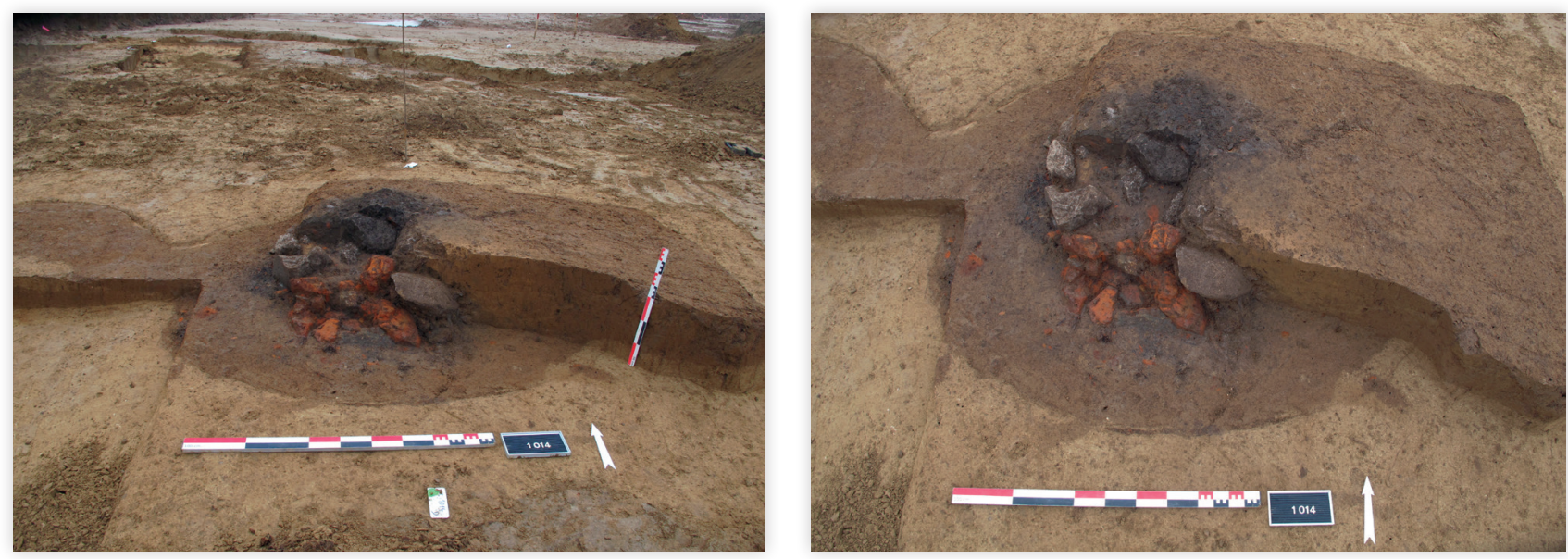
Figure 11

1. Relevé des faits 1013 et 1014 ; 2. Relevé du foyer de Sevaz

(Suisse), d'après Benkert et alii 2010, figure 23.

(c) P. Clerc, P. Girard, M. Michler.
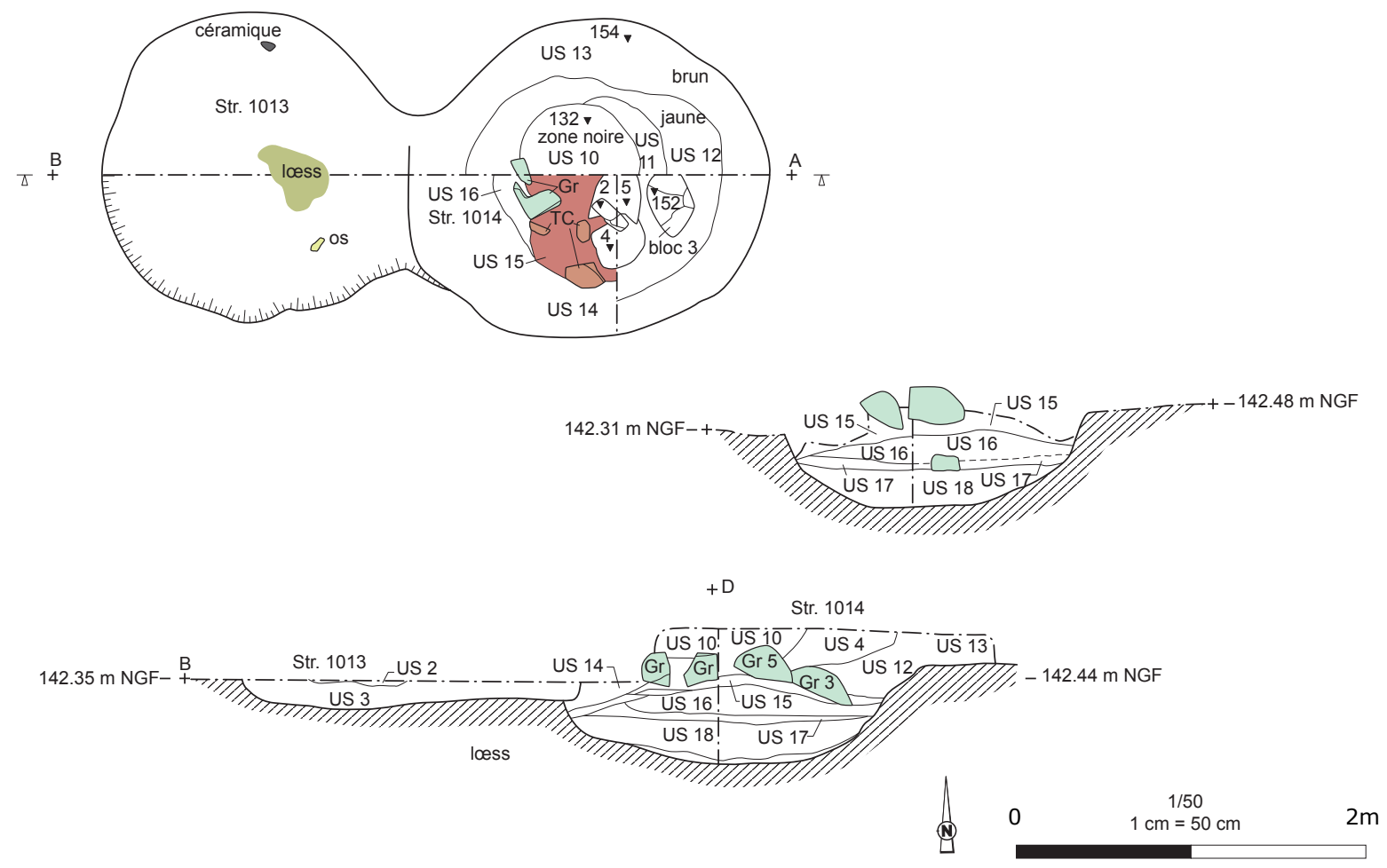

US 2 - limon loessique brun-jaune.

US 3 - limon argileux brun, compact et homogène, avec de très rares micro-charbons de bois, 1 tesson et 1 os.

US 10 - limon argileux brun-noir, compact et hétérogène avec de nombreux fragments de TC et un bloc de granit.

US 11 - limon argileux brun-jaune foncé, compact et homogène, avec présence de charbon de bois, et de taches de TC de très petites dimensions.

US 12 - limon argileux et lœssique jaune, compact et homogène, avec présence de rares charbons de bois.

US 13 - limon argileux brun.

US 14 - limon argileux brun-noir.

US 15 - accumulation de blocs de TC orangée avec localement des traces de vitrification, parfois de plaques de TC et peu de scories en partie centrale et d'avantage sur les bords.

US 16 - limon argileux avec de nombreuses scories sur les parois et des nodules de TC, et présence d'un fragment de céramique.

US 17 - limon argileux noir, compact et hétérogène, très riche en scorie et présence de 2 blocs de granit.

US 18 - lœss brun clair compact et homogène, absence de trace de charbon de bois, de scorie ou de céramique.

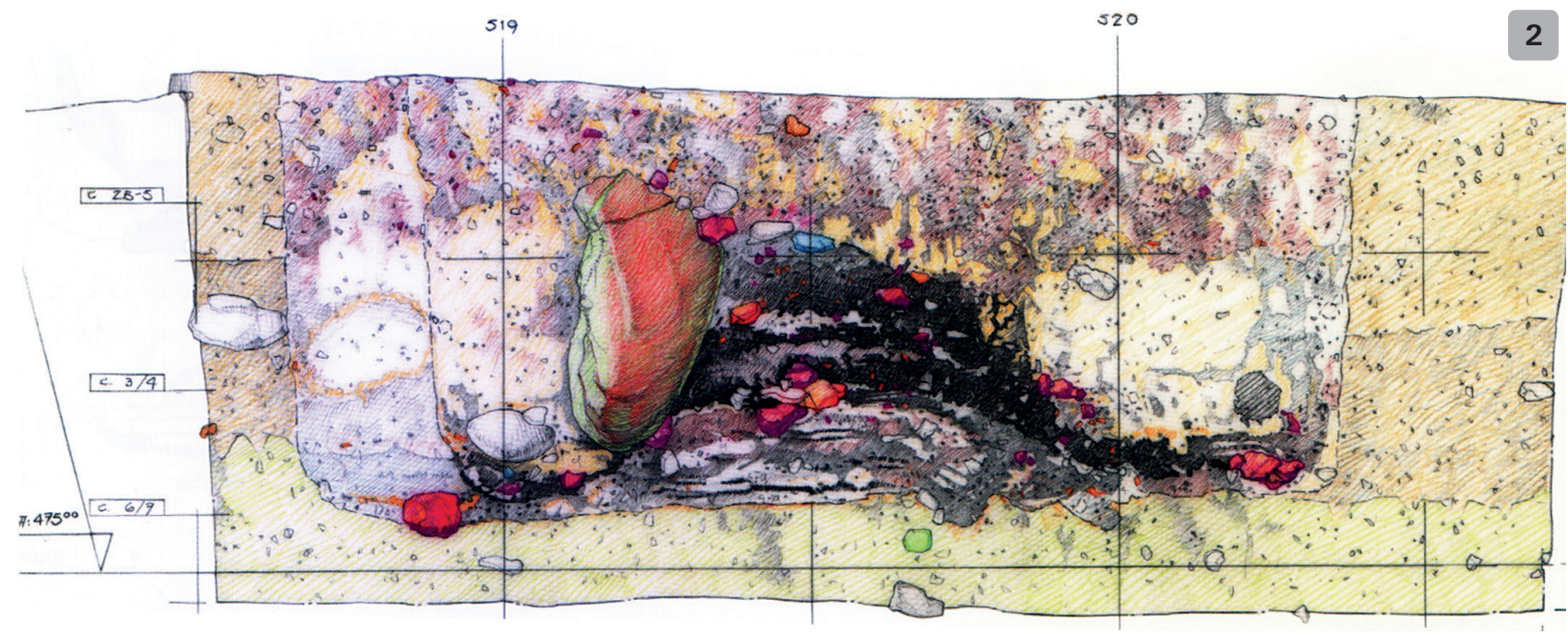




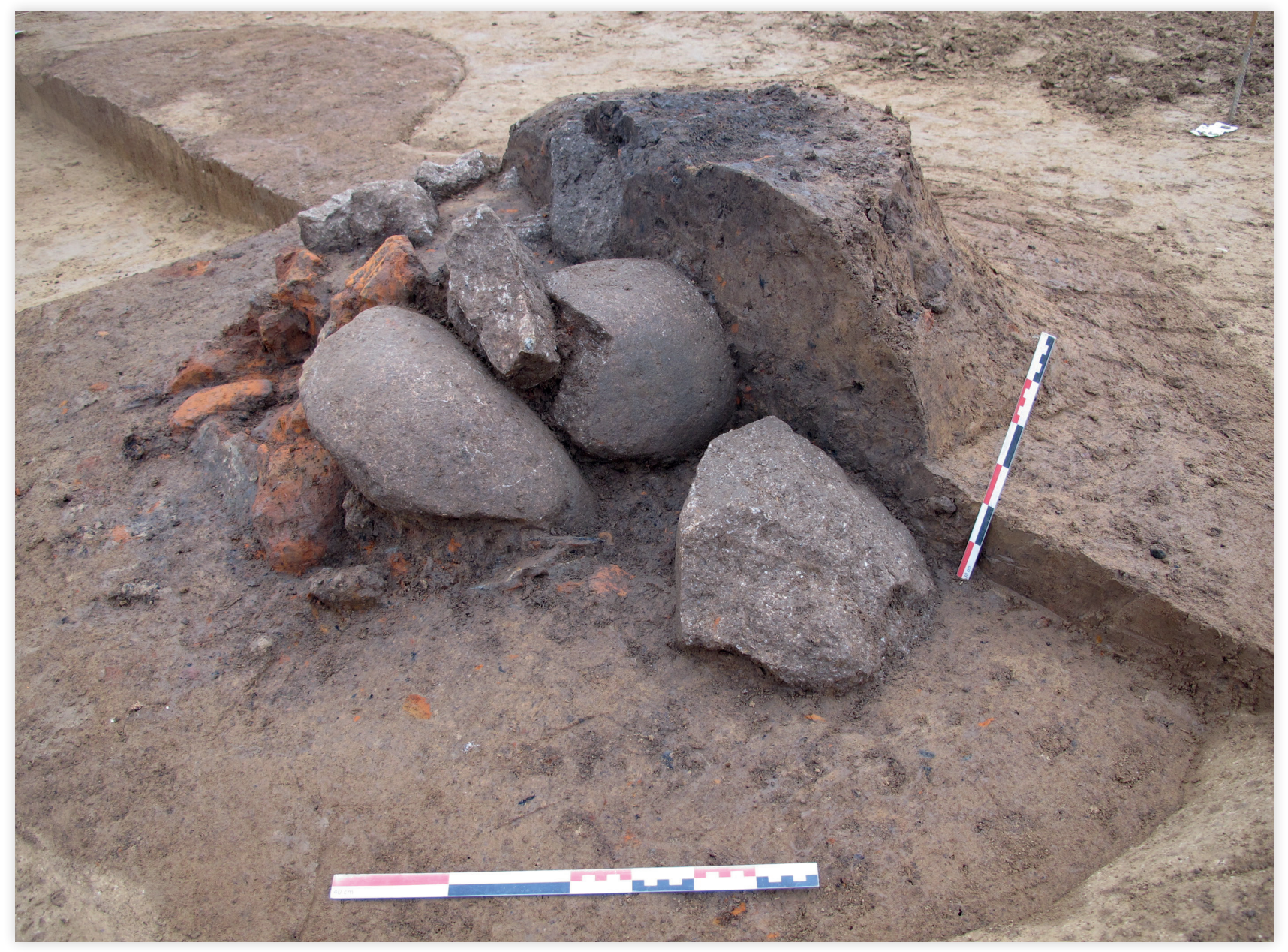

Figure 12

Vue des premiers blocs de granite découverts dans le comblement de la fosse 1014.

(C) cliché P. Clerc, Inrap.

\section{L'ABANDON}

Les niveaux supérieurs de la fosse contiennent et recouvrent les blocs évoqués ci-après. Ils sont pauvres en artefacts et scellent les couches sous-jacentes.

\section{UN LOT EXCEPTIONNEL D'ENCLUMES ABANDONNÉES (FIG, 12)}

La fouille a livré une série de seize blocs granitiques en position secondaire au centre de la structure et reposant sur une couche riche en matériaux archéo-métallurgique.

Certains blocs dépassant les $40 \mathrm{~kg}$ sont intacts, alors que d'autres montrent des cassures relativement fraîches situées sur des zones actives. Ce lot est exceptionnel pour cette période en Europe et illustre les supports de frappe d'un atelier sidérurgique.

[27] BocQUILLON et alii 2009, p. 104, fig. 17.

\section{PLUSIEURS FOYERS DÉMANTELÉS SUR PLACE (FIG. 13)}

Plus de $26 \mathrm{~kg}$ de terre cuite ont été prélevés : parois de foyers de différentes tailles (fig. 13, 1), fragments de tuyères avec conduit de ventilation, bouchons (fig. 13, 2), etc. Des éléments de comparaison sont connus (site contemporain de Vrigny, «Cumines Basses », Marne [27]).

Les foyers dont le dénombrement reste à faire, sont illustrés par des éléments de parois vitrifiées sur près de $2 \mathrm{~cm}$ d'épaisseur. Le plus grand fragment à évent est de forme carrée (20 cm de côté).

Ce niveau correspond à une seule et même phase de comblement, comprenant les résidus de forge et le lot d'enclumes.

\section{UN NIVEAU DE FONCTIONNEMENT ET DES BATTITURES EN QUANTITÉ (FIG. 14)}

Les niveaux sous-jacents composés exclusivement de résidus de forge (scories, petits fragments de terre cuite, parois de foyer, petits blocs granitiques) semblent correspondre au fonctionnement 


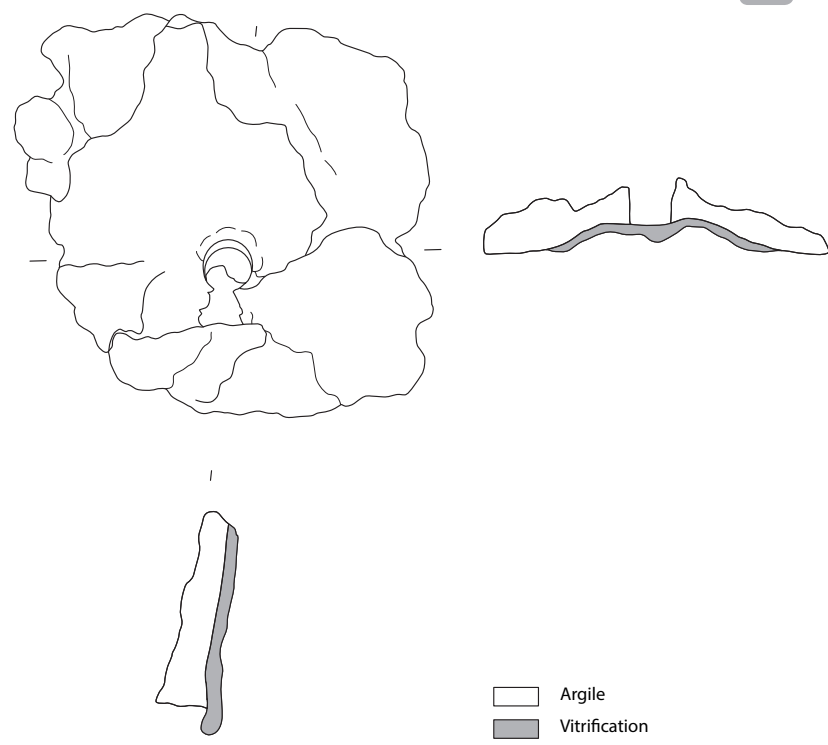

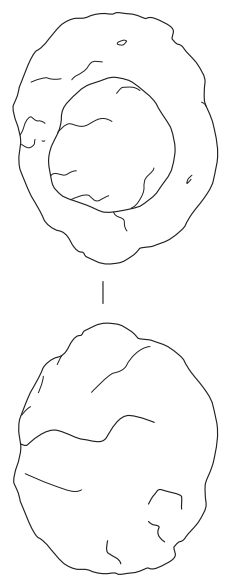

6259-CIN-1014-17-011
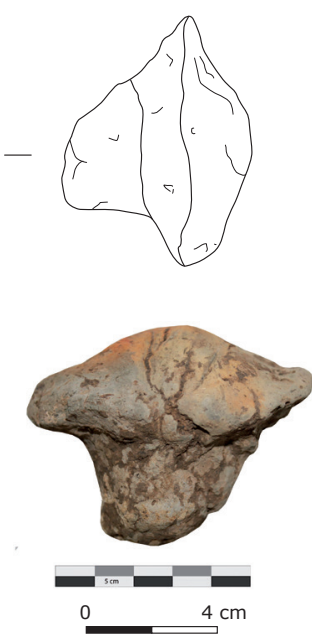

Figure 13

1. Paroi de four en argile cuite avec des traces de vitrification sur la face interne et un évent (fosse 1014, us 15).

2. Bouchon en terre cuite provenant de la fosse 1014.

(c) DAO : Ingrid Turé, Inrap.

Figure 14 : refus de tamis d'un litre de sédiment prélevé dans I'US.16 dans la fosse St.1014.

Parfois jusqu'à 180 gr de battitures par litre.

(C) cliché P. Clerc, Inrap.

d'activités de post-réduction. Les quelques fragments de scories dont la nature peut évoquer des rebuts de réduction sont en quantité trop faible pour pouvoir attester d'une telle activité sur le site (5 fragments pour $558 \mathrm{gr}$ ).

Parmi les scories de post-réduction, les culots de forge, entiers (282 individus) ou fragmentés, sont la catégorie la mieux représentée. Chacun de ces rebuts, de forme plus ou moins hémisphérique, est le produit d'une seule phase du travail

de I'atelier. L'échantillonnage systématique et le tamisage ont permis de quantifier les résidus archéo-métallurgique dont des battitures $(30 \mathrm{~kg})$ de toutes sortes et de toutes les tailles (granulaires, globulaires, lamellaires, billes, etc.) mêlées aux scories. Ces résidus proviennent probablement du nettoyage du niveau de sol de l'atelier (non conservé) en périphérie des enclumes. Les chutes de métal sont rares.

\section{ANALYSES MACROSCOPIQUES DES RÉSIDUS DE LA FORGE (FIG. 15)}

Au total, ce sont près de $70 \mathrm{~kg}$ de scories qui ont été récoltés lors de la fouille, principalement dans le silo 1003 (fouillé de moitié : $23,2 \mathrm{~kg}$ ) et dans la fosse atelier $1014(42,9 \mathrm{~kg})$. Ces déchets sont presque exclusivement des produits issus du fer, de l'allumage à l'extinction du foyer de forge. Selon l'activité en cours (épuration du fer brut, fabrication d'objet, recyclage...) la nature des déchets produits va varier. À Weyersheim, on peut distinguer quatre groupes principaux en fonction de la nature du matériau qui les constitue. Le groupe des scories ferreuses (SFR) est le mieux représenté puisqu'il rassemble plus de $67 \%$ des culots présents sur le site. Ils sont d'une couleur grise à rouille et sont magnétiques, ce qui témoigne de la présence de fer-métal enfermé dans la masse. Il se décline en 2 types : les scories denses (SFR1) et les scories aérées (SFR2). Les scories grises denses (SGD) sont le deuxième groupe distingué. Il rassemble des pièces de couleur sombre et d'une texture compacte et représente $22 \%$ des culots de forge. 
Figure 15

Répartition globale des différents facies de scories.

\begin{tabular}{|c|c|c|c|c|}
\hline Structure & SAS & SFR & SGD & SGC \\
\hline 1002 & & 2 & & 1 \\
\hline 1003 & 10 & 57 & 17 & 5 \\
\hline 1012 & & 1 & & \\
\hline 1014 & 6 & 128 & 45 & 8 \\
\hline 1081 & & 2 & & \\
\hline Total & 16 & 190 & 62 & 14 \\
\hline
\end{tabular}

Nombre de scorie de forge entière par type et par contexte.

\begin{tabular}{|c|c|c|c|c|}
\hline Structure & SAS & SFR & SGD & SGC \\
\hline 1014 & 6 & 128 & 45 & 8 \\
\hline
\end{tabular}

Nombre de scorie de forge entière par type de la fosse 2014.

\begin{tabular}{|c|c|c|c|c|}
\hline Structure & SAS & SFR & SGD & SGC \\
\hline 1003 & 10 & 57 & 17 & 5 \\
\hline
\end{tabular}

Nombre de scorie de forge entière par type du silo 2003.
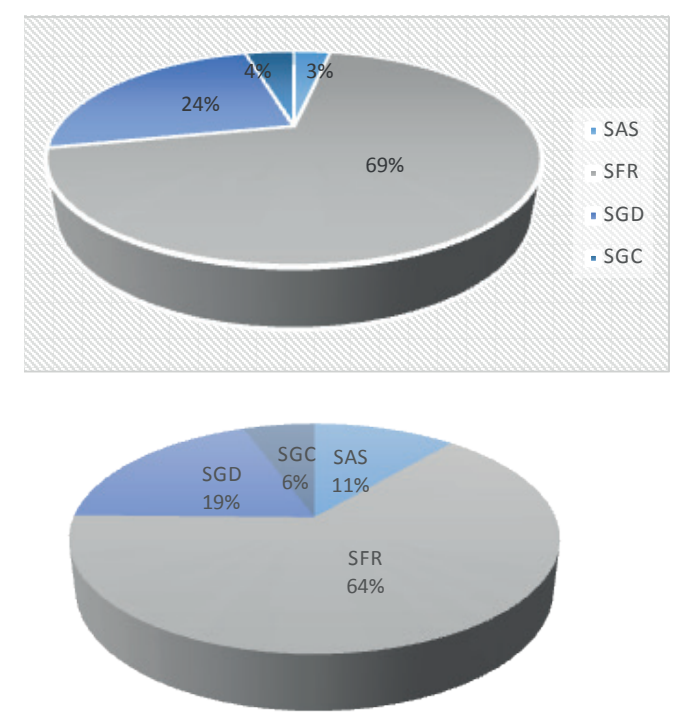

$\because \mathrm{SAS} \backsim \mathrm{SFR} \backsim \mathrm{SGD} \backsim \mathrm{SGC}$

Les scories argilo-sableuses (SAS) sont également divisées en deux types : d'aspect vitreux et de couleur claire (SAS1) et plus denses et plus sombres (SAS2). Seuls 6 culots de forge présentent ce faciès dans l'échantillonnage. Toutefois, une dizaine de coulures de scories indépendantes sont du même matériau (6\%). Le dernier groupe (SGC) rassemble un ensemble de 14 scories grises et charbonneuses (5\%)

La diversité des faciès de scories en culots montre que plusieurs étapes de la chaîne opératoire de la forge ont été pratiquées sur le site, même si les activités produisant des scories SFR sont largement majoritaires.

Au final, la masse des scories de forge des deux structures 1003 et 1014, en ajoutant les éléments contenus dans la seconde moitié du silo, serait d'environ $90 \mathrm{~kg}$. En prenant en compte les battitures et autres micro-déchets de forge, on peut estimer le poids total des déchets compris dans ces 2 structures à $130 \mathrm{~kg}$. Ces valeurs placent le site parmi les plus riches en données pour l'Europe (site de Sévaz en Suisse : $120 \mathrm{~kg}$; Bragny-sur-Saône : $400 \mathrm{~kg}$; Lyon-Vaise : $139 \mathrm{~kg}$ [28]).

[28] FILIPPINI 2015, p. 180-181.

\section{ANALYSES MÉTALLOGRAPHIQUES (FIG. 16)}

Les analyses au microscope optique ont été effectuées au laboratoire Métallurgies et Cultures IRAMAT - URM5060 - CNRS -UTBM par Marion Berranger assistée de Patrick Clerc. À partir d'un échantillonnage représentatif des déchets les plus fréquents l'étude au microscope métallographique a porté sur quinze culots de forge et six chutes métalliques (voir pour la méthodologie Berranger 2014 : 46-47). Les résultats mettent en évidence la pratique de l'intégralité de la chaîne opératoire de post-réduction.

Les cinq culots analysés provenant du silo 1003 sont tous caractéristiques d'une phase d'épuration primaire de masses brutes de réduction (fig. 16A). La proportion de métal perdu au sein des culots est très importante (8 à 14\% des surfaces). Il s'agit de gros fragments de métal mesurant plusieurs centimètres de côté, aux surfaces irrégulières et qui présentent un grand nombre de porosités et de replis non ou peu déformés. Les teneurs en carbone oscillent généralement autour de $0,8 / 0,7 \% \mathrm{C}$ ce qui en faisaient des produits durs, mais parfaitement forgeables. Ces fragments cassants, du fait de leur dureté, se sont détachés de masses de métal brut durant le processus d'épuration. Pour la fosse 1014, I'analyse de dix culots (fig. 16B) et de quatre chutes métalliques 


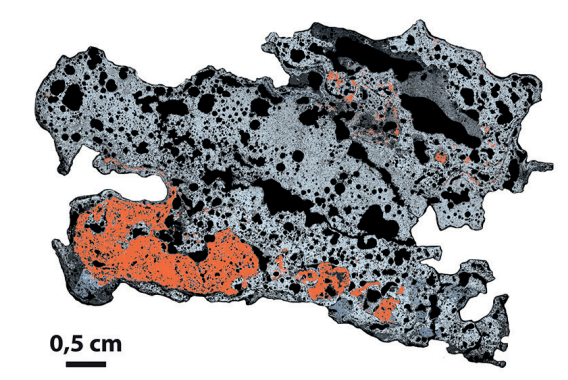

A. Culot SGD 1003-4-9

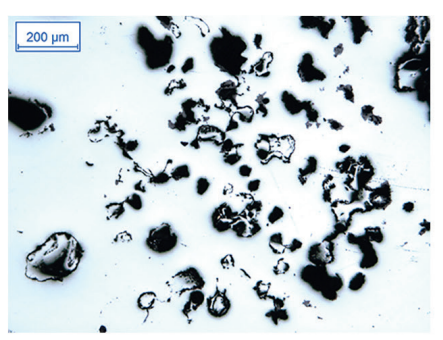

Replis et porosités dans le métal

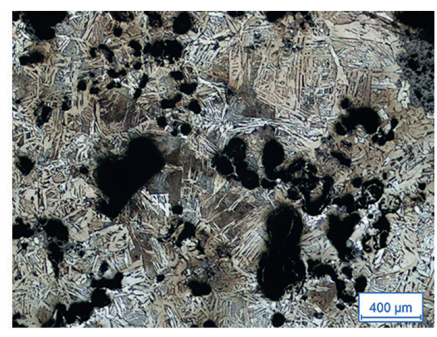

Acier à $0,5 / 0,6 \% C$

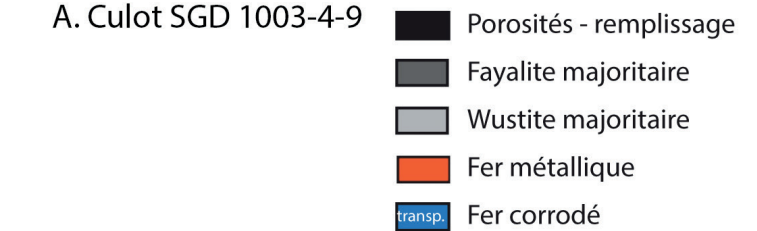

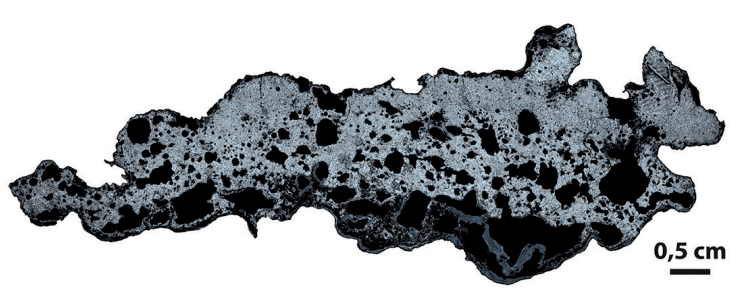

B. Culot SFR2 1014-15-23

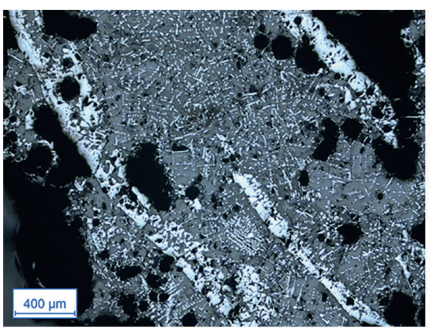

Battitures plates et globulaires dans la matrice base silice

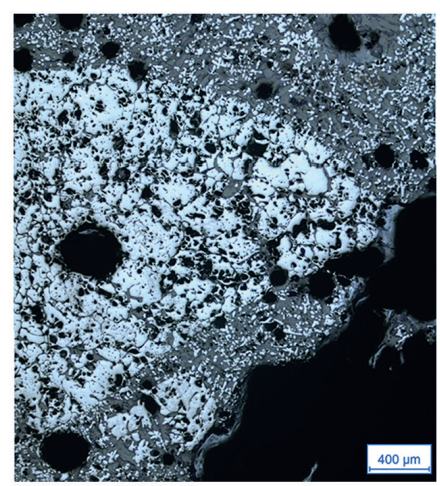

DAO et clichés : M. Berranger - LMC - CNRS

Figure 16

Observations métallographiques sur deux culots issus des fosses 1003 (A) et 1014 (B). À gauche: section polie légendée. À droite : clichés micro-photographiques.

témoigne des étapes de compactage à un stade ultérieur et surtout des étapes de mise en forme d'objets. Dans les culots, la proportion de métal est plus faible que précédemment (inférieure à $1 \%$ des sections) et les surfaces des éclats sont fortement aplanies. Ces culots ont été formés durant des étapes de forte oxydation à chaud ayant entraîné d'abondantes pertes en oxydes (battitures plus ou moins dissoutes). Plusieurs culots se caractérisent par une stratification correspondant à des séquences distinctes d'une même opération technique.

Les déchets métalliques correspondent à des produits assez travaillés, constitués d'acier trempable, aux surfaces fortement déformées et bien épurées. Ces indices permettent de reconnaître le travail d'artisan(s) réalisant des tâches récurrentes et probablement spécialisées, et d'identifier deux types d'activités distinctes, spatialement séparées.

[29] MICHLER et alii, à paraître.

[30] Nous pouvons ajouter à ce lot 73 fragments indéterminés de granite, d'une masse totale de 17,6 kilogrammes, extraits des structures 1003 et 1014 . Tandis que la structure 1003 a livré 9 blocs (2,1 kilogrammes),

\section{OUTILS LITHIQUES LIÉS À LA SPHÈRE MÉTALLURGIQUE}

Il s'agit principalement des enclumes, des percuteurs et des outils d'abrasion pour les polissoirs et les aiguisoirs. Une publication à paraître développera cet aspect [29].

Le lot de 16 blocs de granite, tous brisés, totalisant 206,9 kilogrammes [30], a été choisi parmi de gros blocs roulés (fig. 17).

Les multiples stigmates, sur les faces et les arêtes, supposent différentes étapes de traitement et une utilisation de différents outils à percussion lancée plurifonctionnels [31] (fig. 17, 2 et 3 ). Peu d'enclumes sont connues pour cette période. Citons celle de Sévaz/Tudinges en Suisse [32] sur laquelle est visible une cupule d'origine thermo-mécanique proche de nos surfaces concaves. Pour le $\mathrm{II}^{\mathrm{e}}$ siècle 
Figure 17

1. Dénombrement (graphique radar) des types de surfaces actives, des types d'impacts et des aspects de surfaces présents sur les enclumes découvertes ;

2. Bloc 1 de la fosse 1014. Cette enclume porte une vaste surface active plane, mais interrompue par une cassure ;

3. Bloc 1 de la fosse 1014. Vue avec lumière rasante de la surface active et des impacts nombreux.

4. Graphique des variables issues de l'analyse des correspondances multiples.

(c) cliché P. Clerc, retouche et DAO : M. Michler, Inrap, S. Badey, Inrap.

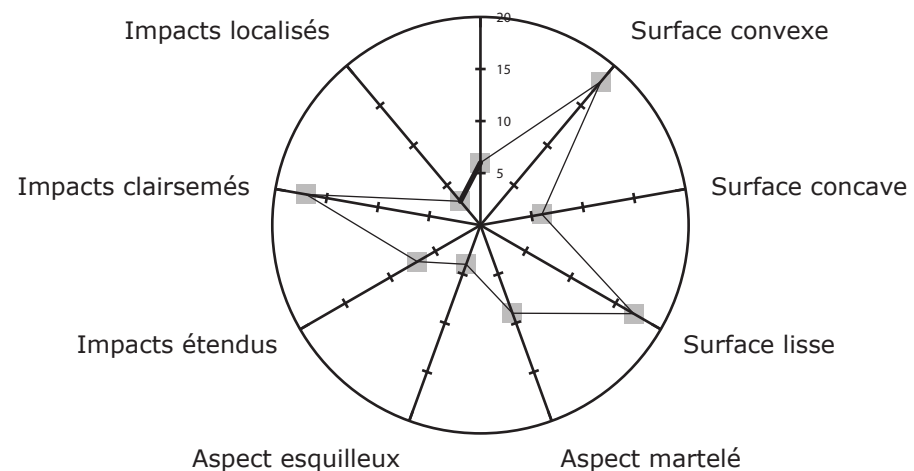

1

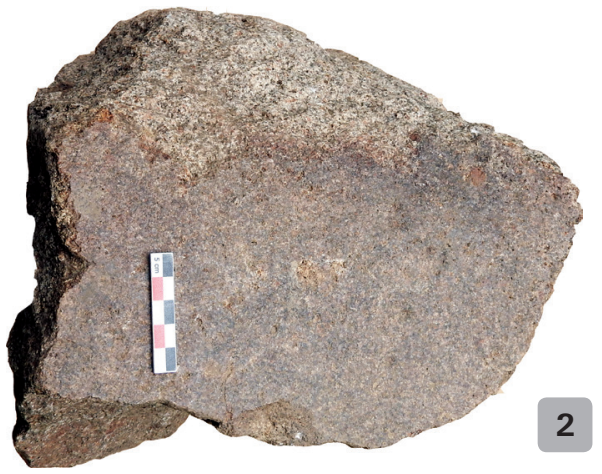

3
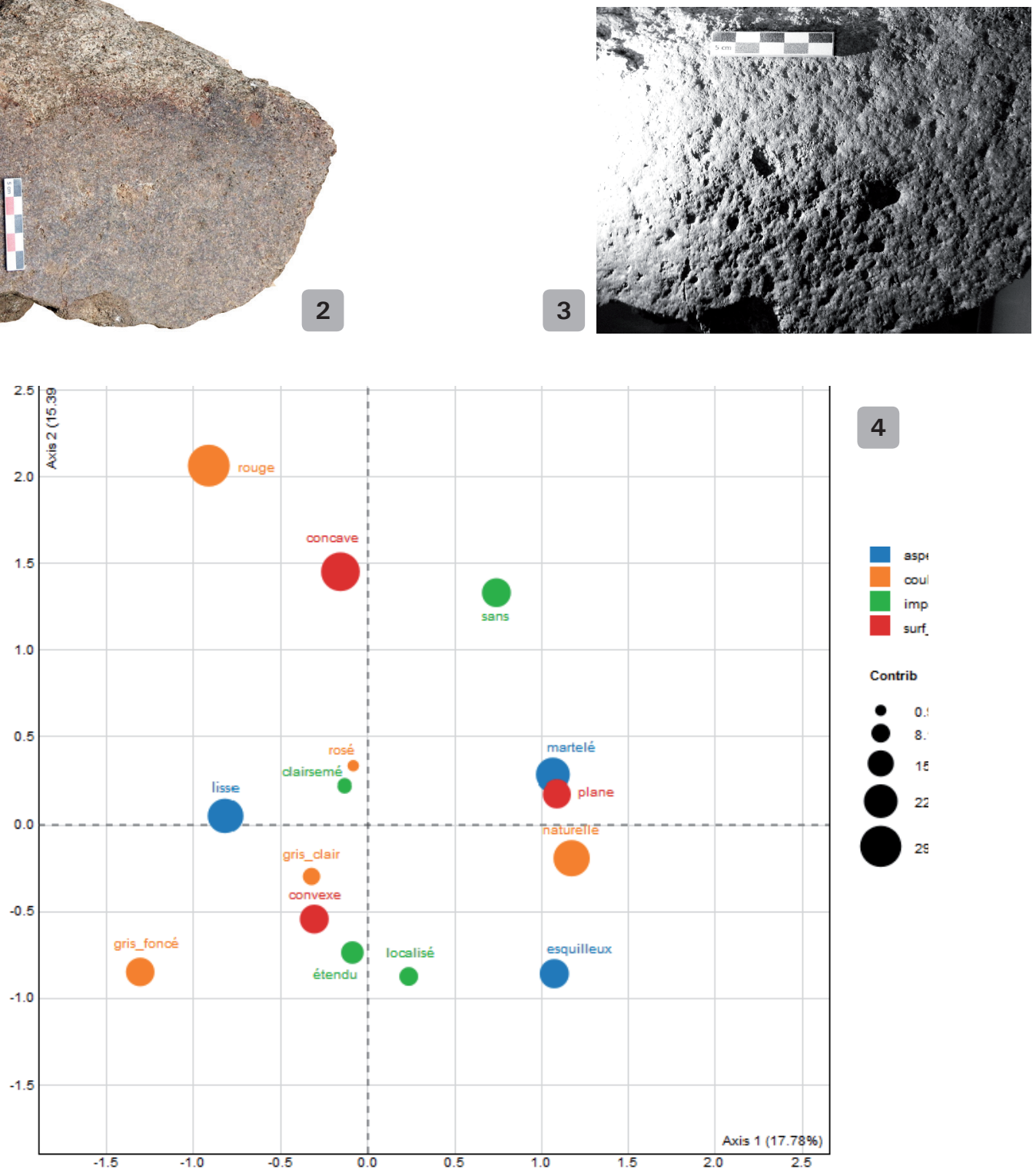
av. J.-C. citons celle de la forge de Paule en Côte d'Armor [33]. Les exemplaires sont aussi rares pour La Tène finale (enclume de Rochères dans I'Aisne, ou de Neuville-sur-Sarthe [34]).

Ces blocs de granite proviennent très probablement de la vallée de la Fecht, à 100 kilomètres au sud-ouest du site de Weyersheim. Cette roche plutonique semble avoir été choisie pour ses propriétés mécaniques. Sa texture est tenace, et par conséquent plus résistante à la propagation d'une fissure [35].

Près de 30 surfaces actives ont été observées sur les galets (fig. 17, 1). Vu l'aspect inédit de cette découverte d'enclume, et en l'absence d'analyses spécifiques sur ce type d'objets, nous avons enregistré plusieurs variables qui nous semblent pertinentes. Ces surfaces sont soit planes, convexes ou concaves, soit lisses, martelées ou esquilleuses. Les impacts peuvent être couvrants, clairsemés, localisés sur de petites zones ou absents. L'absence d'impact ne signifie pas l'absence d'utilisation comme la mise en forme de tôles, peu perceptible. Une coloration rougeâtre et rosée, peut-être induite par le travail de forge, marque également certaines surfaces. La démarche d'analyse statistique a été présentée lors du colloque de Belfast [36] et est disponible dans un rapport de recherche [37].

En définitive cinq groupes de surfaces d'enclumes ont été retenus suite à l'analyse statistique multivariée (fig. 23). Le graphique des variables issu de l'Analyse des Correspondances Multiples (fig. 17, 4) permet de résumer le mieux possible I'information relative aux variables et leurs combinaisons. Soulignons que le plan formé par les deux premiers facteurs ne représente que $33,17 \%$ de I'information, ce qui amène à rester prudent sur les conclusions présentées ci-dessous. Remarquons également que même si la variable impact (sans, clairsemé, étendu, localisé) à des contributions moindres que les autres, il nous a semblé important de la conserver au vu de son importance dans la caractérisation des enclumes.

- La classe 1 regroupe les surfaces actives d'aspect lisse et de couleur grise (clair ou foncé). En outre ces surfaces ont une morphologie convexe (sauf une) et des impacts clairsemés pour la majorité d'entre elles (sauf 3 ). Ces critères nous conduisent à penser que les pièces lithiques ont été employées dans le cadre d'une phase d'élaboration, peut-être pour l'emboutissage de plaques métalliques.

- La classe 4 réunit les surfaces actives martelées (sauf une) et qui présentent des impacts clairsemés (sauf 2). Elles sont soit de couleur naturelle soit gris clair. Par contre elles peuvent présenter une morphologie plane (4) convexe (3), ou concave (2). L'aspect martelé et polymorphe des surfaces actives suggère l'emploi de ces enclumes dans la phase d'épuration. Remarquons également qu'aucune coloration ne teinte la roche. D'après la classification ascendante hiérarchique, cette classe pourrait être divisée en deux groupes. Mais pour le moment, par manque d'éléments de comparaison, cette observation ne dégage pas d'informations pertinentes.

- La classe 3 rassemble les surfaces actives lisses (sauf une) et de couleur rosée. En revanche les surfaces sont planes (2), concaves (1) ou convexes (2) et peuvent présenter tous les types d'impact.

- La classe 5 concentre les surfaces actives convexes, d'aspect esquilleux et de couleur naturelle. Elles comportent des impacts localisés, étendus ou absents.

- La classe 2 assemble les surfaces actives lisses et de couleur rouge. De plus elles peuvent être concaves ou convexes et présenter des impacts clairsemés ou absents.

L'observation attentive des critères regroupés dans les classes 3,5 et 2 permet de nuancer l'organisation de la phase d'élaboration. Tandis que la classe 5 enregistre des marques esquilleuses sur un support plutôt convexe de couleur naturelle, indiquant une frappe plus violente, les deux autres classes présentent des stigmates presque identiques : impacts variés sur un support lisse et polymorphe. Seule la coloration de la surface rosé et rouge - nous indique une légère nuance de chauffe. Ce type de nuance a été observé lors de la chauffe progressive d'échantillons de granite gris réalisée en laboratoire [38]. Les analyses montrent qu'une teinte rougeâtre accompagnée d'un changement de texture apparaît avec l'augmentation de la température, entre $400^{\circ} \mathrm{C}$ et $600^{\circ} \mathrm{C}$.

Tandis que les percuteurs ont pu servir au façonnage et au modelage de pièces métalliques (planage des tôles 1058-1), les polissoirs en grès et aiguisoirs en quartzite (pour les cupro alliages) semblent dédiés à la finition d'objets métalliques.

[33] MeNEZ et alii, p. 224 et 231.

[34] JODRY 2015.

[35] FRONTEAU \& BOYER 2011, p. 113.

[36] MICHLER et alii à paraître.

[37] BADEY 2016.

[38] Vasquez et alii, 2016. 


\section{Hierarchical Clustering}
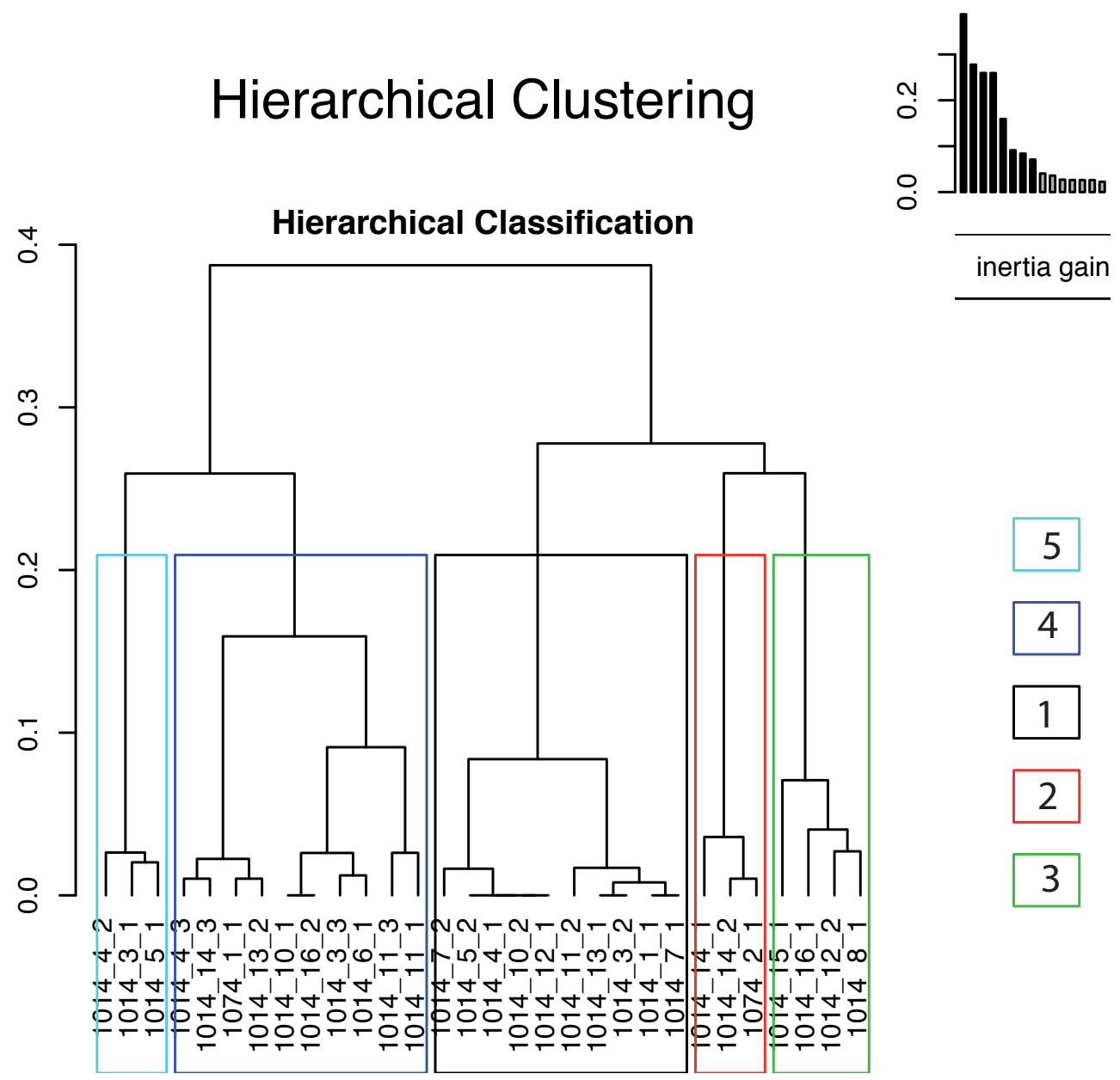

Figure 18

3

Dendrogramme

issu du

classement

ascendant

hiérarchique.

(C) Sylvain

Badey, Inrap.

\section{CONCLUSIONS}

À I'heure actuelle l'activité métallurgique sur le site de Weyersheim «Les Hauts de la Zorn », constitue un cas unique en Alsace de par la quantité hors-norme de déchets métallurgiques découverts $(110 \mathrm{~kg})$.

L'analyse des fosses circulaires 1003 et 1014 suggère un fonctionnement probablement synchrone. La fosse 1014 a livré le plus d'informations, avec la mise en évidence d'un premier niveau de fonctionnement et deux couches d'abandon de I'atelier [39]. Quant aux enclumes elles portent différents types d'impacts et de surfaces actives correspondant probablement à deux types de travail de la forge d'épuration et d'élaboration. L'étude des culots et déchets métalliques permet d'identifier les mêmes types d'activités. Lors de la fouille de la fosse 1014, le sédiment rempli de déchets (battitures, restes de foyers) a été prélevé. Seule une partie de ces restes a été étudiée pour le moment. Morphologiquement, la fosse 1014 présente les mêmes caractéristiques que celles où sont implantés des ateliers contemporains. Deux fosses circulaires sont ainsi connues sur le site de Sévaz [40] (fig. 11, 2) ou une fosse ronde rue du Docteur Horand à Lyon [41].

L'examen d'une partie des culots suppose la réalisation de deux activités distinctes : épuration d'un métal brut ou peu épuré (st. 1003) et mise en forme d'objets au niveau de l'atelier (st. 1014). En retenant I'hypothèse de contemporanéité, la localisation de ces activités dans une même zone montre une organisation précise et complémentaire des ateliers de forgeage. Dans le cas d'une succession assez courte dans le temps, il faudrait souligner l'emprise forte de l'activité métallurgique dans ce secteur du site. Il manque les traces de l'atelier d'épuration d'où proviennent les culots rejetés dans le silo 1003. Ce dernier se trouve probablement à proximité, mais hors emprise de la fouille. Une prospection géophysique serait à engager pour localiser les restes de cet atelier plus à l'ouest dans les parcelles encore en culture.

[39] Cf. supra.

[40] MAUVILLY et alii, 2007, fig. 2, structures 1 et 2. [41] FiLiPINI 2015, p. 43. 
Soulignons qu'aucun objet achevé ne provient de la fosse 1014 qui n'a livré que des chutes, sous forme de barres, tiges ou tôle. L'herminette, la lance et le ciseau proviennent de silos situés dans la zone d'ensilage et au nord. Leur fabrication sur place n'est pas établie et la question de leur origine reste ouverte. Quoi qu'il en soit leurs formes s'apparentent avec celles d'exemples connus à la même période dans le domaine hallstattien centre-occidental.

Afin de replacer le site de Weyersheim dans son contexte régional, un tableau synthétique, faisant le bilan des découvertes contemporaines en Alsace a été réalisé (fig. 19). Sur ces sites les scories semblent essentiellement résulter d'une activité de forge. Dans certains cas, l'imprécision de l'identification ne permet pas de différencier les déchets de réduction, d'épuration ou d'élaboration. Une reprise des données semble nécessaire.

Une grande majorité des sites recensés présente à la fois des vestiges du travail du fer et des alliages cuivreux. C'est également le cas à Weyersheim. Cette association est courante pour cette période entre le Hallstatt final et La Tène ancienne [42].

Weyersheim se distingue par contre clairement des autres sites par la quantité importante de matériel lié à la métallurgie, tous types confondus (sauf pour les creusets). La découverte d'un niveau de fonctionnement d'atelier constitue d'ailleurs une nouveauté dans la région. Le second ensemble important correspond au site d'Entzheim-Geispolsheim fouillé sur près de sept hectares cumulés en 2006 et 2008-2009. Sur ce site, les restes liés à la métallurgie sont majoritairement en position secondaire comme c'est le cas pour les autres petits sites mentionnés.

Soulignons également le fait que ces traces d'activités métallurgiques se situent toujours à proximité d'une zone d'ensilage, plus rarement à proximité d'un habitat identifié. Le cas de l'atelier isolé est pour le moment inconnu. L'association entre activités métallurgiques et stockage de denrées en silo est particulièrement notable à Weyersheim. On peut même ici évoquer une centralisation de la production agricole au vu du nombre de silos identifiés (une cinquantaine). Ce lien entre métallurgie et zone de stockage a déjà été mis en évidence et étudié en Lorraine à partir des sites de Gondreville «ZAC de la Roseraie et Velaine-en-Haye » et «ZAC Herbue Chalin » [43] situés à proximité de I'habitat fortifié de la «Cité d'Afrique » de Messein. Dans ce dernier cas, un lien de dépendance entre I'habitat privilégié et les établissements ruraux de type fermes-greniers est envisagé, de manière concomitante au contrôle de la production du fer [44].

Plus largement, au niveau national et européen, les synthèses récentes sur le sujet [45] soulignent le nombre limité de gisements à vocation métallurgique (23 recensés dans la thèse $d^{\prime} A$. Filippini) et la faible proportion de déchets retrouvés. Quelques ensembles font exception par la masse de scories présentes tels celui de Bragnysur-Saône « Sous-Moussières » [46] qui livre plusieurs centaines de kilogrammes de déchets. Le site de Sévaz «Tudingues » en Suisse est l'un des rares qui puisse être comparé à Weyersheim par les proportions similaires de déchets de travail du fer (120 kg de scories [47]). Néanmoins, ces sites se distinguent de celui de Weyersheim par la présence de céramique tournée et/ou d'objets d'importation évoquant un statut social élevé des habitants. À Weyersheim la céramique tournée est absente, mais cela pourrait peut-être être lié à la chronologie, cette céramique apparaissant plutôt à La Tène $\mathrm{A} 1$ et étant très peu représentée à la phase antérieure [48]. La datation du site étant assez large (problème du plateau du Hallstatt pour les datations ${ }^{14} \mathrm{C}$ ) cette hypothèse n'est pas à exclure.

L'observation de la carte des principaux sites datés entre le Hallstatt D3 et La Tène ancienne présentant des traces de métallurgie dans le nord Alsace (fig. 2 et fig. 19) montre une concentration d'une vingtaine de kilomètres de diamètre constitué des sites d'Ettendorf, Mittelhausen, Souffelweyersheim, Brumath et Weyersheim, situé le plus haut au nord). À I'heure actuelle, de telles découvertes n'ont pas été observées au delà d' Haguenau. Il reste encore à déterminer comment la production d'objets en fer était organisée du point de vue régional dans le complexe hallstattien occidental. En cette fin de premier âge du Fer, la concentration du pouvoir et des richesses aux mains d'une élite sociale, occupant le plus souvent des centres fortifiés (Illfurth, « Britzgyberg » et Breisach, « Münsterberg ») est à prendre en compte. Dans la forêt de Haguenau, de riches sépultures sont connues à la fin du Hallstatt, mais un net appauvrissement est perceptible au

[42] FILIPPINI 2015.

[43] LeRoY \& CABBoÏ (dir), 2013.

[44] BERRANGER et alii 2017.

[45] FILIPPINI 2015.

[46] FLOUEST 2007.

[47] MAUVILLY et alii 2007.

[48] AdAM et alii 2011. 
Figure 19

Inventaire des sites alsaciens ayant livré des indices de travail du fer.

(C) M. Michler, Inrap.

\begin{tabular}{|c|c|c|c|c|c|c|}
\hline \multirow{3}{*}{$\begin{array}{c}\text { Bruebach «Rennwasen» } \\
\text { et «Auf dem Burner } \\
\text { (H.-R.) }\end{array}$} & \multicolumn{2}{|c|}{ Culots de forge/scories } & \multicolumn{2}{|c|}{ Creusets/Moules } & \multicolumn{2}{|c|}{ Parois de four vitrifiées } \\
\hline & Présence & $\mathrm{X}$ & Présence & $x$ & Présence & $\mathrm{x}$ \\
\hline & NR & $?$ & NR & & NR & \\
\hline \multirow{4}{*}{$\begin{array}{l}\text { Datation : Ha D3-LT A1 } \\
\text { Type de site : silos prospection/ } \\
\text { fouille } \\
\text { Surface en } \mathrm{m}^{2}: \text { / }\end{array}$} & Masse $(\mathrm{kg})$ & $?$ & Masse $(\mathrm{kg})$ & & Masse $(\mathrm{kg})$ & \\
\hline & \multicolumn{2}{|c|}{ Battitures } & \multicolumn{2}{|c|}{ Chute de métal } & \multicolumn{2}{|c|}{ Bibliographie } \\
\hline & Présence & & Présence & & \multirow{2}{*}{\multicolumn{2}{|c|}{ Koenig et al 2005}} \\
\hline & Infos & & NR chute & & & \\
\hline
\end{tabular}

\begin{tabular}{|c|c|c|c|c|c|}
\hline \multirow{3}{*}{$\begin{array}{l}\text { Brumath Lotissement Manet } \\
\text { (B.-R.). }\end{array}$} & \multicolumn{2}{|c|}{ Culots de forge/scories } & Creusets/Moules & \multicolumn{2}{|c|}{ Parois de four vitrifiées } \\
\hline & Présence & fragments & Présence & Présence & Possible tuyère ? \\
\hline & NR & & NR & NR & \\
\hline \multirow{4}{*}{$\begin{array}{l}\text { Datation : Ha D1-D2 } \\
\text { Type de site : petit habitat } \\
\text { ouvert } \\
\text { Surface en } \mathrm{m}^{2} \text { : env. } 10000\end{array}$} & Masse $(\mathrm{kg})$ & & Masse $(\mathrm{kg})$ & Masse $(\mathrm{kg})$ & \\
\hline & \multicolumn{2}{|c|}{ Battitures } & Chute de métal & \multicolumn{2}{|c|}{ Bibliographie } \\
\hline & Présence & & Présence & \multirow{2}{*}{\multicolumn{2}{|c|}{$\begin{array}{l}\text { Mentele, Plouin, Guillaume } 2005, \\
\text { p. } 158 \text {, pl. } 2,19\end{array}$}} \\
\hline & Infos & & NR chute & & \\
\hline
\end{tabular}

\begin{tabular}{|c|c|c|c|c|c|c|}
\hline \multirow{3}{*}{$\begin{array}{l}\text { Ettendorf «Gaentzbruch» } \\
\text { (B.-R.) }\end{array}$} & \multicolumn{2}{|c|}{ Culots de forge/scories } & \multicolumn{2}{|c|}{ Creusets/Moules } & \multicolumn{2}{|c|}{ Parois de four vitrifiées } \\
\hline & Présence & $x$ & Présence & $x$ & Présence & $x$ \\
\hline & NR & 8 & NR & 1 & NR & 11 \\
\hline \multirow{4}{*}{$\begin{array}{l}\text { Datation : Ha D2-LT B1 } \\
\text { Type de site : aire d'ensilage } \\
\text { Surface en } \mathrm{m}^{2}: 2000\end{array}$} & Masse $(\mathrm{kg})$ & 1,65 & Masse $(\mathrm{kg})$ & 0,013 & Masse $(\mathrm{kg})$ & \\
\hline & \multicolumn{2}{|c|}{ Battitures } & \multicolumn{2}{|c|}{ Chute de métal } & \multicolumn{2}{|c|}{ Bibliographie } \\
\hline & Présence & $x$ & Présence & $x$ & \multirow{2}{*}{\multicolumn{2}{|c|}{$\begin{array}{c}\text { Peytremann, } 2004, \text { p. } 25-26, \\
\text { fig. } 15\end{array}$}} \\
\hline & Infos & 0,854 & NR chute & $1 /$ & & \\
\hline
\end{tabular}

Geispolsheim «Schwobenfeld» (B.-R.)

/ Entzheim «in der Klamm»

Datation : Ha D3-LTA et LT A-B1 Type de site : aire d'ensilage avec traces d'habitat

Surface en $\mathrm{m}^{2}: 7$ ha au total

\begin{tabular}{|c|c|c|c|c|c|}
\hline \multicolumn{2}{|c|}{ Culots de forge/scories } & \multicolumn{2}{|c|}{ Creusets/Moules } & \multicolumn{2}{|c|}{ Parois de four vitrifiées } \\
\hline Présence & $x$ & Présence & $x$ & Présence & $x$ \\
\hline NR & 32 & NR & & NR & \\
\hline Masse $(\mathrm{kg})$ & 14 & Masse $(\mathrm{kg})$ & & Masse $(\mathrm{kg})$ & 13,5 \\
\hline \multicolumn{2}{|c|}{ Battitures } & \multicolumn{2}{|c|}{ Chute de métal } & \multicolumn{2}{|c|}{ Bibliographie } \\
\hline Présence & $x$ & Présence & $x$ & \multirow{2}{*}{\multicolumn{2}{|c|}{ Landolt 2007}} \\
\hline Infos & dans 55 str. & NR chute & 1 & & \\
\hline
\end{tabular}

Illfurth «Britzgyberg», «Buergelen», «Naegelberg» (H.-R.)

Datation : Ha D3-LT A1

Type de site : site de hauteur et habitat

Surface en $\mathrm{m}^{2}$ : plus de 4000

\begin{tabular}{|c|c|c|c|c|c|}
\hline \multicolumn{2}{|c|}{ Culots de forge/scories } & \multicolumn{2}{|c|}{ Creusets/Moules } & \multicolumn{2}{|c|}{ Parois de four vitrifiées } \\
\hline Présence & $x$ & Présence & ? & Présence & \\
\hline NR & & NR & & NR & \\
\hline Masse $(\mathrm{kg})$ & & Masse $(\mathrm{kg})$ & & Masse $(\mathrm{kg})$ & \\
\hline \multicolumn{2}{|c|}{ Battitures } & \multicolumn{2}{|c|}{ Chute de métal } & \multicolumn{2}{|c|}{ Bibliographie } \\
\hline Présence & & Présence & & \multirow{2}{*}{\multicolumn{2}{|c|}{ Adam et alii, 2011, p. 65-67 }} \\
\hline Infos & & NR chute & & & \\
\hline
\end{tabular}


Kutzenhouse lotissement Galgenstraenge IV (B.-R.)

Datation : âge du fer ? Type de site : silo, diag Surface en $\mathrm{m}^{2}$ : 1000

\begin{tabular}{|c|c|c|c|c|c|}
\hline \multicolumn{2}{|c|}{ Culots de forge/scories } & \multicolumn{2}{|c|}{ Creusets/Moules } & \multicolumn{2}{|c|}{ Parois de four vitrifiées } \\
\hline Présence & $\mathrm{X}$ & Présence & Présence & X \\
\hline NR & 2 & NR & & NR & 6 , orifice tuyère \\
\hline Masse $(\mathrm{kg})$ & 1,3 & Masse $(\mathrm{kg})$ & & Masse $(\mathrm{kg})$ & \\
\hline \multicolumn{2}{|c|}{ Battitures } & Chute de métal & \multicolumn{2}{|c|}{ Bibliographie } \\
\hline Présence & Présence & $X$ & \multicolumn{2}{|c|}{ Latron 2015 } \\
\hline Infos & NR chute & 2 & \multicolumn{2}{|c|}{} \\
\hline
\end{tabular}

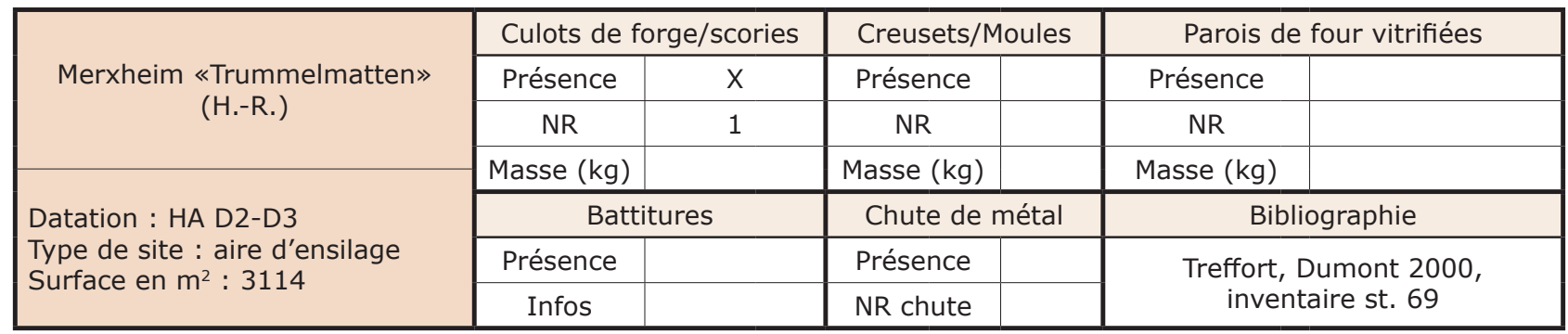

Mittelhausen «Liesbuehl / imbretter Weg»

(B.-R.)

Datation : LTA

Type de site : aire d'ensilage Surface en $\mathrm{m}^{2}: 5000$

\begin{tabular}{|c|c|c|c|c|c|}
\hline \multicolumn{2}{|c|}{ Culots de forge/scories } & \multicolumn{2}{|c|}{ Creusets/Moules } & \multicolumn{2}{|c|}{ Parois de four vitrifiées } \\
\hline Présence & $\mathrm{X}$ & Présence & $\mathrm{X}$ & Présence & X \\
\hline NR & 22 & NR & & NR & 0,99 \\
\hline Masse $(\mathrm{kg})$ & 0,574 & Masse $(\mathrm{kg})$ & 0,44 & Masse $(\mathrm{kg})$ & \multicolumn{2}{|c|}{ Bibliographie } \\
\hline \multicolumn{2}{|c|}{ Battitures } & \multicolumn{2}{|c|}{ Chute de métal } & \multicolumn{2}{|c|}{ Croutsch et al. 2015} \\
\hline Présence & $\mathrm{X}$ & Présence & \multicolumn{2}{|c|}{} \\
\hline Infos & $?$ & NR chute & \multicolumn{2}{c|}{} \\
\hline
\end{tabular}

\begin{tabular}{|c|c|c|c|c|c|c|}
\hline \multirow{3}{*}{$\begin{array}{l}\text { Souffelweyersheim } \\
\text { «Les Sept Arpents» } \\
\text { (B.-R.) }\end{array}$} & \multicolumn{2}{|c|}{ Culots de forge/scories } & \multicolumn{2}{|c|}{ Creusets/Moules } & \multicolumn{2}{|c|}{ Parois de four vitrifiées } \\
\hline & Présence & $x$ & Présence & $x$ & Présence & \\
\hline & NR & 2 & NR & 5 & NR & \\
\hline \multirow{4}{*}{$\begin{array}{l}\text { Datation : LT A2 } \\
\text { Type de site : petit habitat } \\
\text { ouvert } \\
\text { Surface en } \mathrm{m}^{2}: 5000\end{array}$} & Masse $(\mathrm{kg})$ & & Masse $(\mathrm{kg})$ & & Masse $(\mathrm{kg})$ & \\
\hline & \multicolumn{2}{|c|}{ Battitures } & \multicolumn{2}{|c|}{ Chute de métal } & \multicolumn{2}{|c|}{ Bibliographie } \\
\hline & Présence & $x$ & Présence & & \multirow{2}{*}{\multicolumn{2}{|c|}{$\begin{array}{l}\text { Lefranc et alii, 2008, p. 56-59, } \\
\text { pl. } 11, \text { n}^{\circ} 11\end{array}$}} \\
\hline & Infos & $?$ & NR chute & & & \\
\hline
\end{tabular}

Weyersheim Les Hauts de la Zorn (B.-R.)

Datation : Ha D3-LT A1 Type de site : aire d'ensilage avec rares traces $d$ 'habitat Surface en $\mathrm{m}^{2}$ : env. 10000

\begin{tabular}{|c|c|c|c|c|c|}
\hline \multicolumn{2}{|c|}{ Culots de forge/scories } & \multicolumn{2}{c|}{ Creusets/Moules } & \multicolumn{2}{|c|}{ Parois de four vitrifiées } \\
\hline Présence & $\mathrm{X}$ & Présence & $\mathrm{X}$ & Présence & $\mathrm{X}$ \\
\hline NR & & $\mathrm{NR}$ & 0,1 & NR & 30 \\
\hline Masse $(\mathrm{kg})$ & 67 & Masse $(\mathrm{kg})$ & & Masse $(\mathrm{kg})$ & \multicolumn{2}{|c|}{ Bibliographie } \\
\hline \multicolumn{2}{|c|}{ Battitures } & \multicolumn{2}{c|}{ Chute de métal } & \multicolumn{2}{|c|}{ Michler et alli, à paraître } \\
\hline Présence & $\mathrm{X}$ & Présence & $\mathrm{X}$ & \multicolumn{2}{|c|}{} \\
\hline Infos & $\begin{array}{c}35 \mathrm{~kg} \text { esti- } \\
\text { més }\end{array}$ & NR chute & 27 & \multicolumn{2}{|c|}{} \\
\hline
\end{tabular}

Wolfgantzen Deviation RN415 (H.-R.)

Datation : Ha D3-LT A1

Type de site : petit habitat ouvert

Surface en $\mathrm{m}^{2}: 7000$

\begin{tabular}{|c|c|c|c|c|}
\hline \multicolumn{2}{|c|}{ Culots de forge/scories } & \multicolumn{2}{|c|}{ Creusets/Moules } & \multicolumn{2}{|c|}{ Parois de four vitrifiées } \\
\hline Présence & $\mathrm{X}$ & Présence & Présence & \\
\hline NR & 1 & NR & NR & \\
\hline Masse $(\mathrm{kg})$ & & Masse $(\mathrm{kg})$ & Masse $(\mathrm{kg})$ & \multicolumn{2}{|c|}{ Bibliographie } \\
\hline \multicolumn{2}{|c|}{ Battitures } & Chute de métal & \\
\hline Présence & Présence & \\
\hline Infos & NR chute & \\
\hline
\end{tabular}


Hallstatt D3 alors qu'à La Tène A/B une prospérité persistante est à souligner [49]. Pour le moment, la proposition de voir dans le site du Hexenberg, proche de la forêt de Haguenau, au nord-est de Weyersheim, une possible résidence princière n'a pas été confirmée, les fouilles sur le site se rattachant plutôt au Bronze final et au Hallstatt C [50]. Il est possible que ce site de statut élevé se trouve ailleurs ou que le modèle des territoires princiers soit à nuancer dans ce secteur. Quoi qu'il en soit la région de Haguenau, de par sa position près du Rhin, reste un point de relais et de contrôle indéniable [51]. Les mêmes questionnements ont été évoqués pour la production primaire de fer et le rôle de cette activité dans le développement des centres de pouvoir au cours du premier âge du Fer [52]. Pour le moment, au vu de l'indigence des découvertes la question reste ouverte.

Concernant le type de matière première employée sur le site de Weyersheim, les analyses ont montré ce minerai était encore relativement brut. Son origine reste pour le moment inconnue. Il faut de plus noter l'absence d'indices de réduction connus dans la plaine rhénane. Du côté allemand, en ForêtNoire, il faut aller à une soixantaine de kilomètres à vol d'oiseau de Weyersheim, pour trouver un centre de production du fer, dans le secteur de Neuenbürg [53]. À la fin de la période hallstattienne et au début de La Tène, ce district minier compte plus de soixante-dix lieux de chauffe, dont des bas fourneaux, ce qui suggère une exploitation « proto-industrielle» du secteur.

Soulignons également que Weyersheim se trouve à une trentaine de kilomètres de la vallée de la Murg qui sépare deux sites de hauteur (Battert et
Neuenbürg) et correspondant à un passage entre Forêt-Noire et Alsace [54]. Ne négligeons pas non plus une origine depuis des sites de production locaux plus modestes. Les demi-produits bipyramidaux de fer connus à cette période et bien attestés en Alsace [55] ont également pu constituer une source d'approvisionnement. La découverte récente de plusieurs dizaines de demi-produits bipyramidés à Durrenentzen, non loin du site «princier » de Breisach, souligne l'importance de ce type d'objet dans la circulation du métal sous forme brute en Alsace au ve siècle av. J.-C. [56].

L'intérêt du site de Weyersheim est divers. En effet, rares sont les sites de ces périodes livrant de telles quantités de déchets de production du fer se rattachant de plus au traitement du fer depuis sa forme brute jusqu'à l'élaboration d'objets. L'association entre activités métallurgiques et zone de stockage de denrées agricoles ouvre également un large champ de réflexion sur les conditions de production et de diffusion de ces biens. Il reste à présent à mieux déterminer la place exacte et le rôle tenu par ces ateliers dans les réseaux de production et d'échange du métal en Alsace du Nord au début du second âge du Fer.

[49] Koenig \& LeGENDRE 1997, p. 90.

[50] Ibid, p. 90 ; LASSERRE et alii 2001.

[51] KoENIG \& LEGENDRE 1997, p. 90.

[52] DiSSER 2014, p. 500 ; DILLMANN et alii 2017.

[53] GASSMANN et alii 2006 et 2014.

[54] GenTNER 2016, p. 160.

[55] BERRANGER 2014.

[56] KUHNLE 2015. 
AChARD-Corompt, Nathalie \& Riquier, Vincent, 2013, Chasse, culte ou artisanat ? Les fosses «à profil en $Y-V-W \gg$. Structures énigmatiques et récurrentes du Néolithique aux âges des Métaux en France et alentours, Actes de la table ronde de Châlons-en-Champagne, 15/16 nov. 2010, Dijon (RAE suppl. 33).

Adam, Anne-Marie, Defressigne, Sylvie, Koenig, Marie-Pierre \& Lasserre, Marina 2011, avec la contribution d'Aurore Boyer, Hélène Delnef, Michaël Landolt, Suzanne Plouin, Murielle Roth-Zehner, \& Nicolas Tikonoff et la collaboration d'Inès Balzer, Gertrud Kuhnle, Jean-Michel Treffort \& Cécile Véber, La céramique d'habitat du Bronze final IIIb à La Tène B en Alsace et en Lorraine. Essai de typo-chronologie, Dijon (RAE suppl. 29).

Auxiette, Ginette, Baudry, Anna \& Meniel Patrice, 2010, « Une histoire de l'élevage dans l'ouest de la Normandie : les sites de Mondeville, Ifs, Fleury, Creully (Calvados) et les autres » dans Barral Philippe Dedet, Bernard Delrieu Fabien, Giraud Pierre, Le Goff Isabelle, Marion Stéphane, Villard-Le Tiec Anne (éd.), L'Âge du fer en BasseNormandie, Actes du XXXIII colloque international de I'AFEAF ; Caen, 20 au 24 mai 2009, Besançon (Annales littéraires de l'Université de Franche-Comté n 883 ; Série « Environnement, sociétés et archéologie »), p. 185-202.

BADEY, Sylvain, 2016, Analyse statistique des surfaces actives d'enclumes de Weyersheim, rapport de recherche, disponible en ligne : https://halshs.archives-ouvertes.fr/halshs-01436440.

BAUvAIS, Sylvain, 2008, «Du prestige à la proto-industrie : évolution des pratiques sidérurgiques au second âge du Fer dans le Nord du Bassin Parisien », The Arkeotek Journal 2, 4 ; www.thearkeotekjournal.org.

Benkert, Alain, Curdy, Philippe, Epiney-Nicoud, Claire, Kaenel, Gilbert, Mac Cullough, Fiona, Mauvilly, Michel \& RuffieuX, Mireille 2010, « Zentralisierungprozess und Siedlungdynamik in der Schweiz », dans D. Krausse (éd.), « Fürstensitze » und Zentralorte der frühen Kelten, Abschlusskolloquium des DFG-Schwerpunktprogramms 1171 in Stuttgart, 12.-15. Oktober 2009, Teil I, Landesamt für Denkmalpflege im Regierungspräsidium Stuttgart, Stuttgart (Forschungen und Berichte zur Vor- und Frühgeschichte in BadenWürttemberg 120), p. 79-118.

BerRANGer, Marion, 2014, Le fer, entre matière première et moyen d'échange, en France, du viI $I^{e}$ au I ${ }^{\text {er }}$ siècle avant J.-C. : approches interdisciplinaires, Dijon (Collection Art, Archéologie et Patrimoine).

Berranger, Marion, Zaour, Nolwenn, Leroy, Marc, Bauvais, Sylvain, Cabboï, Luisella \& Dunikowski, Christophe, 2017, «Organisation des productions sidérurgiques en Gaule (VII ${ }^{\mathrm{e}} \mathrm{I}^{\mathrm{er}}$ siècle av. J.-C.) : de la réduction du métal à l'élaboration des demi-produits » dans Production et proto-industrialisation aux âges du Fer, Actes du 39 e colloque de I'AFEAF tenu à Nancy du 14 au 17 mai 2015, Bordeaux (Mémoires 47), p. 301-334.

Bocquillon, Hervé, SAURel Marion, Dunikowski, Christophe \& Yvinec, Jean-Hervé, 2009 , «abitats et zones d'activités à Vrigny (Marne) à la fin du premier âge du Fer », dans Jan Vanmoerkerke (dir.), Le bassin de la Vesle du Bronze final au Moyen Age, Bulletin de la société archéologique champenoise 102, 2, p. 82-152.

Boutorlle, Linda, 2015, «Les techniques du dinandier de l'âge du Bronze : I'outillage en pierre spécifique à la déformation plastique des métaux », dans Nicolas Théophane et Boulud-Gazo Sylvie (éd.), Artisanats et productions à l'âge du Bronze, Actes de la journée de la Société Préhistorique Française, Nantes, 2011 (Séance de la Société Préhistorique Française 4), p. 83-96.

BROC, Émeline, 2011, Initiation aux méthodes de l'archéozoologie : Étude de deux sites du Bas-Rhin, Mittelhausen et Meistratzheim, Mémoire de Master, dactylographié, Université de Bourgogne, Dijon.

Croutsch, Christophe, 2012, Mittelhausen, Bas-Rhin, «Liesbuehl, Gimbretter Weg », construction de la LGV Est Européenne Tronçon $H$ - Site 10-1. Structures domestiques et funéraires du Michelsberg et de La Tène, Rapport de fouille, PAIR.

Croutsch, Christophe, Landolt Michaël, Broc, Émilie, Fleischer, Félix, Mulot, Agathe, Nocus Noémie, Pélissier Amélie, Putelat Olivier \& Rousselet Oriane, 2015, « Le site de Mittelhausen «Liesbuehl / Gimbretter Weg » (Bas-Rhin, Alsace) : économie et environnement d'une aire d'ensilage de La Tène ancienne dans le Kochersberg », Revue Archéologique de I'Est 64, 2015, p. 111-160.

DABECK, Pierre, 2013, Weyersheim (Bas-Rhin), Rue de la Gare, lotissement «les Hauts de la Zorn », Indices de l'âge du Bronze et occupation du Hallstatt final/La Tène ancienne. Rapport de diagnostic, Inrap Ges.

DEFFRESSIGNE, Sylvie, 2017, «La place des productions au sein des habitats du bassin de Nancy entre le VIII ${ }^{\mathrm{e}}$ et le ve s. av. J.-C. », dans Marion Stéphane, Defressigne Sylvie, Kaurin Jenny \& Bataille Gérard (éd.), Production et proto-industrialisation aux âges du Fer, perspectives sociales et environnementales, XXXIXe colloque AFAEF, Nancy 14-17 mai 2015, Bordeaux (Mémoire 47, suppl. 14/2), p. 649-674.

Deffressigne, Sylvie \& LANdolt, Michaël, 2017, « L'évolution du stockage entre XI ${ }^{\mathrm{e}}$ et le III $^{\mathrm{e}}$ siècle av. J-C. dans les vallées de la Moselle, de la Meurthe et du Rhin », dans Marion, Stéphane, Defressigne Sylvie, Kaurin Jenny \& Bataille Gérard (éd.), Production et proto-industrialisation aux âges du Fer, perspectives sociales et environnementales, XXXIXe colloque AFAEF, Nancy 14-17 mai 2015,. Bordeaux (Mémoire 47, suppl. 14/2), p. 77-100.

Deffressigne, Sylvie \& Leroy, Marc, 2013 « L'organisation de la production du fer au cours de l'âge du Fer dans le bassin médian de la Moselle », dans Leroy Marc \& Cabboï Luisella (dir.), Les formes d'organisation de la production du fer en métallurgie anciennes. Systèmes de production et chaînes opératoires dans les ateliers de l'est du Bassin parisien de l'âge du Fer au haut Moyen Âge, rapport de PCR, p. 41-78. 
Deffressigne, Sylvie, Tikonoff, Nicolas, Boulanger-Boucher, Karine, Chaussé, Christine \& TesnierHermetey, Corinne, 2002, « Les gisements d'habitat de la fin du Premier âge du Fer à Gondreville-Fontenoy-surMoselle (54). Le stockage intensif et ses conséquences économiques et sociales », Archaeologia Mosellana 4, p. 81-184. Dillmann, Philippe, Arandra, Régis, L'Héritier, Maxime \& Fluzin, Philippe, 2005 : « Forging of ironsteel artefacts: archaeology, experiments and archaeometry », dans M. Urteaga (éd.), Iron, History and Heritage. 1st International Conference of Paleosiderurgy and Industrial Heritage Recovery, San Sebastian, p. 311-324.

Dillmann, Philippe, Schwab, Roland, Bauvais, Sylvain, Brauns, Michael, Disser, Alexandre, Leroy, Stéphanie, Gassmann, Guntram \& Fluzin, Philippe, 2017, « Circulation of iron products in the North-Alpine area during the end of the first Iron Age $\left(6^{\text {th }}-5^{\text {th }}\right.$ C. BC): A combination of chemical and isotopic approaches», Journal of Archaeological Science 87, p. 108-124.

Dunikowski, Christophe \& Саввої, Sandra, 1995, La sidérurgie chez les Sénons : les ateliers celtiques et gallo-romains des Clérimois (Yonne), Paris (Documents d'Archéologie Française 51).

Disser, Alexandre, 2014, Production et circulation du fer en Lorraine (VI ${ }^{e}$ s. av. J.-C. - $x V^{e}$ s. ap. J.-C.), Thèse de doctorat, Université de Belfort-Montbéliard.

DubreucQ, Émilie, 2013, Métal des premiers Celtes : productions métalliques sur les habitats des provinces du Hallstatt centre-occidental, Dijon (Art, archéologie et patrimoine).

Filippini, Anne, 2015, La métallurgie du fer dans le centre-est de la France au ve siècle avant J.-C. Approches historique, archéologique et archéométrique, Tours (59e supplément à la Revue archéologique du Centre de la France).

FLouest, Jean-Loup, 2007, «Approches quantitatives de la production de fer sur le site hallstattien de Bragnysur-Saône (Saône-et-Loire) » dans Pierre-Yves Milcent, (éd.), L'économie du fer protohistorique : de la production à la consommation du métal. XXVIIIe colloque AFAEF, Toulouse 20-23 mai 2004, Bordeaux (Aquitania, suppl. $14 / 2)$, p. 265-270.

FronteAu Gilles \& Boyer, François, 2011, « Les géomatériaux meuliers : de l'identification des sources géologiques à la définition de catégories de gisements », dans Olivier Buchsenschutz, Luc Jaccottey, Florent Jodry \& JeanLuc Blanchard (éds), Évolution typologique et technique des meules du Néolithique à l'an mille sur le territoire français. IIIe Rencontres Archéologiques de l'Archéosite gaulois, Bordeaux (Aquitania, suppl. 23), p. 121-135.

Gassmann, Guntram, Rösch, Manfred \& Wieland, Günther, 2006, « Das Neuenbürger Erzrevier im Nordschwarzwald als Wirtschaftsraum während der Späthallstatt- und Frühlatènezeit », Germania 84, p. 273-306.

GASSMANN, Guntram \& Wieland, Günther, 2014, « Frühkeltische Eisenproduktion im Nordschwarzwald: Montanarchäologische Forschungen im Neuenbürger Erzrevier 2004-2011 », dans S. Hornung (éd.), Produktion Distribution - Ökonomie, Siedlungs- und Wirtschaftsmuster der Latènezeit, Akten des internationalen Kolloquiums in Otzenhausen, 28.-30. Oktober 2011, Bonn (Universitätsforschungen zur prähistorischen Archäologie 258), p. 163-170. Gentner, Steeve, 2016, «Économie du fer et voies de communication de l'abattage du minerai à la distribution du métal : I'exemple du nord de la Forêt-Noire au ve siècle avant J.-C. », Archimède 3, p. 150-168.

Hamon, Caroline \& Blanchet, Stéphane, 2015, « Le macro-outillage lithique sur les sites de l'âge du Bronze armoricain : quelques hypothèses fonctionnelles pour aborder la notion d'artisanat », dans Nicolas Théophane \& Sylvie Boulud-Gazo (éd.), Artisanats et productions à l'âge du Bronze, Actes de la journée de la Société Préhistorique Française, Nantes, 2011 (Séance de la Société Préhistorique Française 4), p. 63-82.

JODRY, Florent, 2015, «Étude des enclumes de Neuville-sur-Sarthe-La Chataigneraie », dans Jean-Yves Langlois (éd.), Le bassin sidérurgique du nord du Mans de l'âge du Fer au Moyen Âge. Rapport de fouille, Inrap Grand-Ouest.

Koenig, Marie-Pierre \& LegendRe, Jean-Pierre, 1997, « Une "résidence princière" au Hallstatt D dans la région de Haguenau », dans Patrice Brune \& Bruno Chaume (dir.), Vix et les éphémères principautés celtiques, Actes du Colloque de Chatillon-sur-Seine, Paris (Archéologie Aujourd'hui), p. 83-92.

KuhNLe, Gertrud \& Tesnier-Hermetey, Corinne 2005, « Holtzheim « Am Schluesselberg 》 (Bas-Rhin) : un habitat hallstattien de bord de terrasse loessique dans la plaine du Rhin supérieur », dans Anne-Marie Adam (dir.), Recherches de Protohistoire alsacienne : la céramique d'habitat du Bronze final III à La Tène ancienne, Dijon (23ème supplément à la Revue Archéologique de l'Est), p. 179-209.

Kunnle, Gertrud, dir., 2015, Un deuxième dépôt de demi-produits de fer de l'âge du Fer à Durrenentzen, Rapport de diagnostic archéologique, Inrap Grand Est Sud, Strasbourg.

LAbAUne, Régis \& Alix, Stéphane, 2014, Talant, Plombières-les Dijon, Côte-d'Or, Bourgogne, Peute Combe, Les Vaux Bruns : découverte d'un établissement rural gallo-romain et d'un hameau à vocation artisanale du ve siècle avant J. C. Rapport de fouilles, Inrap GES, Dijon, 6 vol.

Landolt, Michaël, Van Es, Marieke, Putelat, Olivier, Bouquin, Denis, SchaAl, Caroline \& BoËs, Éric, 2007, Entzheim-Geispolsheim (67) : Aéroparc (Lidl-CUS), les occupations protohistoriques. Rapport de fouille, Pôle d’Archéologie Interdépartemental Rhénan, Sélestat.

LANdolt, Michaël, Abert, Franck, Bolly, Alexandre \& LeProvost, Céline, 2013, Entzheim-Geispolsheim, Bas-Rhin, Lotissement d'activités du quadrant 4, Entzheim «In der Klamm » et Geispolsheim "Schwobenfeld », Rapport de fouille préventive, Pôle d'Archéologie Interdépartemental Rhénan, Sélestat, 6 vol.

Lasserre, Marina, Vigreux, Thomas, Basoge, florian, Logel, Thierry, Putelat, Olivier, Schneider, Nathalie, Michler, Matthieu, Jodry, Florent \& BoËs, Xavier, 2011, « Le site de la fin du Bronze final du Hexenberg à Leutenheim (Bas-Rhin) : études sur le paléoenvironnement rhénan et études archéologiques. Résultats préliminaires », Bulletin de la Société Préhistorique Française 108, p. 731-754.

Latron-Colecchia, Anna Maria, 2015, Quelques indices matériels de la Culture Bischheim occidentale du Rhin supérieur et des témoins d'une activité métallurgique de l'âge du Fer à Kurtzenhouse lotissement Galgenstraenge IV, Rapport de diagnostic archéologique, Inrap, GES, Strasbourg. 
Lefranc, Philippe, BoËs, Éric \& Véber, Cécile, 2008, « Un habitat de La Tène ancienne à Souffelweyersheim «Les Sept Arpents » (Bas-Rhin)», Revue archéologique de I'Est 57, p. 41-74.

Leroy, Marc \& CAвboï, Sandra (dir), 2013, Les formes d'organisation de la production du fer en métallurgie ancienne. Systèmes de production et chaînes opératoires dans les ateliers de l'est du Bassin parisien de l'âge du Fer au haut Moyen Âge, Projet collectif de recherche, SRA Lorraine, Jarville.

LeUvReY, Jean-Michel, 1999, Hauterive-Champréveyres, 12. L'industrie lithique du Bronze final, étude typo-technologique, Neuchâtel (Archéologie neuchâteloise 24).

Mauvilly Michel, Serneels, Vincent, Ruffieux, Mireille \& Garcia-Cristobal, Evencio, 2007, « Le travail du fer dans une forge du milieu du ve siècle av. J.-C. à Sévaz-Tudingues (canton de Fribourg, Suisse) », dans Pierre-Yves Milcent (dir.), L'économie du fer protohistorique : de la production à la consommation du métal, XXVIII colloque AFEAF, Toulouse, 2004, Bordeaux (Aquitania Supplément 14-2), p. 271-278.

Malrain, François, Blancquert, Geertrui \& LoRhi, Thierry, 2013, L'habitat rural du second âge du Fer. Rythmes de création et d'abandon au nord de la Loire, Paris (Recherches Archéologiques 7).

Menez, Yves, Viver Jean-Bernard, Chanson, Karine \& Dupré, Mathilde, 2007, « La forge de Paule (Côtesd'Armor) », dans Pierre Yves Milcent (dir.), L'économie du fer protohistorique : de la production à la consommation du métal, XXVIII colloque AFEAF, Toulouse 2004, Bordeaux (Aquitania Supplément 14-2), p. 213-237.

MÉniel, Patrice, 1993, «La faune des structures de La Tène ancienne de Rosheim « Mittelweg » et « Sandgrube » », dans Christian Jeunesse, Patrice Méniel \& Brigitte Röder (éd.), L'habitat de La Tène ancienne de Rosheim « Mittelweg » (Bas-Rhin). Fouilles 1992, Strasbourg (Monographies d'archéologie alsacienne 1), p. 132-149.

Mentele, Serge, Plouin, Suzanne \& Guillaume, Maxime 2005, « L'habitat hallstattien de Brumath « lotissement Édouard Manet-deuxième tranche » (Bas-Rhin)», dans Anne-Marie Adam (dir.), Recherches de Protohistoire alsacienne : la céramique d'habitat du Bronze final III à La Tène ancienne, Dijon (23e supplément à la Revue Archéologique de l'Est), p. 143-178.

MichleR, Matthieu, 2016, Ensilage et artisanat du fer au Hallstatt D3/La Tène ancienne à Weyersheim, Rapport de fouille, Inrap GES, Strasbourg.

Michler, Matthieu, Jodry, Florent, Badey, Sylvain \& Clerc, Patrick, avec la coll. de BerRANGer, Marion, CABboï, Luisella \& VAN Es, Marieke, à paraître, « La forge hallstattienne de Weyersheim (Bas-Rhin, France) - Hallstatt D3/La Tène ancienne », dans Linda Boutoille, Rebecca Peake (éd.), Le métallurgiste et ses outils : symboliques, fonctions et techniques durant l'âge du Bronze et l'âge du Fer, Actes du colloque UISPP à Belfast, 24-25 juin 2016.

Pieters, Maxence, 2013, Les outils comme traceurs des activités de transformation des métaux ? Supports de frappe, abrasifs et brunissoirs, outils d'aiguisage et outils de broyage, Thèse de doctorat, Université de Bourgogne, Dijon.

Putelat, Olivier, 2007, « La faune », dans Michaël Landolt (dir.), Entzheim-Geispolsheim (Alsace, Bas-Rhin), Aéroparc (LIDL-CUS), Rapport de fouille préventive, vol. 3, Les occupations protohistoriques, Sélestat, Pôle d'Archéologie Interdépartemental Rhénan.

Putelat, Olivier, 2010, « Archéozoologie » dans Carine Deslex \& Philippe Amiot (dir.), Occupations protohistoriques à Chevenez : de l'âge du Bronze à la fin de l'âge du Fer, Porrentruy (Cahier d'archéologie jurassienne 26), p. 52, 77-82, 113-119, 139-153.

Putelat, Olivier, 2015, Les relations homme-animal dans le monde des vivants et des morts. Étude archéozoologique des établissements et des regroupements funéraires ruraux de l'Arc jurassien et de la Plaine d'Alsace, de la fin de I'Antiquité tardive au premier Moyen Âge, Thèse d'archéologie, Université de Paris 1 Panthéon-Sorbonne.

Putelat, Olivier, 2016, « Étude de la faune », dans Michler, Matthieu, Ensilage et artisanat du fer au Hallstatt D3/La Tène ancienne à Weyersheim, 2016, p. 161-179

Putelat, Olivier \& LANdolt, Michaël, 2013, « La caractérisation des dépôts animaux de La Tène ancienne à Geispolsheim Schwobenfeld 》(Bas-Rhin), dans Ginette Auxiette \& Patrice Méniel (dir.), Les dépôts d'ossements d'animaux en France, de la fouille à l'interprétation, Actes de la table-ronde de Bibracte, 15-17 octobre 2012, Montagnac (Archéologie des plantes et des animaux 4), p. 25-68.

ROth-Zehner, Muriel, 2013, « Les établissements ruraux de la fin du Hallstatt et du début de La Tène dans la plaine d'Alsace. État de la question », Cahiers Alsaciens d'Archéologie d'Art et d'Histoire 56, p. 53-87.

Treffort, Jean-Michel \& Dumont, Annie, 2000, Merxheim «Trummelmatten», Néolithique, Bronze final, Hallstatt et haut Moyen Âge, Document final de synthèse de sauvetage urgent, A.F.A.N., Strasbourg.

Vazquez, Patricia, Acuña, Marta, Benavente, David, Gibeaux, Soizic, Navarro, Irene \& GomezHerAs, Miguel, 2016, «Evolution of surface properties of ornamental granitoids exposed to high temperatures », Construction and Building Materials 104, p. 263-275.

VÉbER, Cécile, 2015, Une occupation du Bronze moyen, des silos de La Tène ancienne et un chemin médiéval. Duntzenheim, Bas-Rhin, «Schlittweg, Weingartenberg», LGV EE site 8-2. Rapport de fouilles, Inrap GES, Strasbourg. Zenner, Muriel, 2001, Hatten, zone industrielle, lieu-dit Rothsmatt (Bas-Rhin). Document final de synthèse, fouille de sauvetage urgent, Antea. 


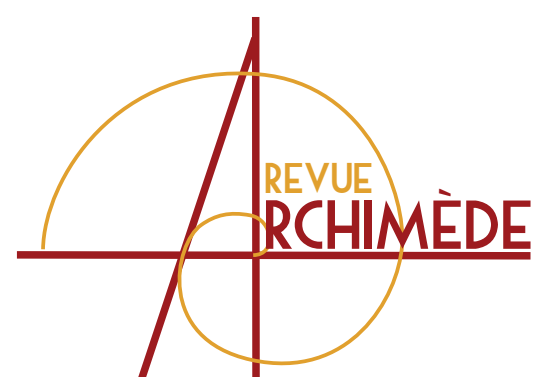

ARCHÉOLOGIE ET HISTOIRE ANCIENNE

\title{
LE TERRITOIRE DE WARCQ À L'ÉPOQUE ROMAINE : RÉSULTATS DE SIX FOUILLES PRÉVENTIVES (2012-2017) MENÉES DANS LA PARTIE NORD DU DÉPARTEMENT DES ARDENNES, PRÈS DE CHARLEVILLE-MÉZIĖRES
}

\author{
Gael CARTRON \\ Archéologue au bureau d'études Éveha \\ UMR 7041 ArScAn \\ gael.cartron@eveha.fr \\ Bertrand ROSEAU \\ Archéologue au bureau d'études Éveha \\ EA 1132 Hiscant - MA \\ bertrand.roseau@eveha.fr
}

RÉSUMÉ

Depuis quelques années, la recherche archéologique a bénéficié du suivi systématique, effectué sous la forme de diagnostics, des nombreux projets d'aménagements qui intéressent la commune de Warcq (08), nous fournissant une vision de I'occupation du sol jusqu'alors insoupçonnée. Six fouilles préventives y ont été entreprises depuis 2012, toutes centrées sur des vestiges antiques, nous permettant d'agrandir notre échelle d'analyse tout en fournissant des données très précises et variées, par exemple sur la morphologie des occupations. Située sur des terrains fertiles près de la Meuse, à quelques kilomètres d'agglomérations antiques identifiées sur les communes de Charleville-Mézières et de Ville-sur-Lumes, cette

MOTS-CLÉs

Ardennes,

Warcq,

époque romaine, archéologie préventive, voie antique,

établissement rural, thermes. portion du territoire ardennais est aujourd'hui la mieux documentée par l'archéologie préventive dans un périmètre de plusieurs dizaines de kilomètres.
Julien BRUYÈRE

Archéologue au conseil départemental des

Ardennes

julien.bruyere@cd08.fr

Over the past few years, archaeological research has benefited from the systematic surveillance of numerous development projects in the municipality of Warcq (08), which have provided an unprecedented vision of land use. Five rescue excavations, all focusing on Roman remains, have been organized since 2012, improving the scope of our analysis while providing both very specific and varied data, for instance on site morphology. Situated on fertile soils near the Meus, a few kilometres away from the urbanised Roman sites found within the limits of the towns of Charleville-Mézières and Ville-Sur-Lumes, this portion of the Ardennes is today the most well-documented archaeological area within a radius of several dozen kilometres.
KeYWORDS Ardennes department, Warcq, Roman period, preventive archaeology, Roman way, rural settlement, thermae. 


\section{INTRODUCTION}

Le présent article propose un premier bilan des fouilles menées ces dernières années par la cellule d'archéologie préventive du département des Ardennes sur la commune de Warcq (fig. 1), immédiatement à I'ouest de Charleville-Mézières (08). Il fait suite à un article rédigé à l'issue des campagnes de diagnostics, qui visait avant tout à attirer l'attention sur le potentiel archéologique de cette zone, particulièrement riche en vestiges des premiers siècles de notre ère [1]. Ce territoire est aujourd'hui I'un des mieux documentés du département dans un périmètre de plusieurs dizaines de kilomètres ( 81 hectares sondés à ce jour dans cette commune, soit $8,8 \%$ de sa superficie totale). La recherche archéologique a notamment bénéficié des suivis effectués préalablement aux travaux de construction ou d'aménagement concernant le village actuel ou, plus récemment, de ceux réalisés plus à I'ouest en amont de projets industriels. Ces terrains,

Figure 1

Localisation de la commune de Warcq (C CD 08).

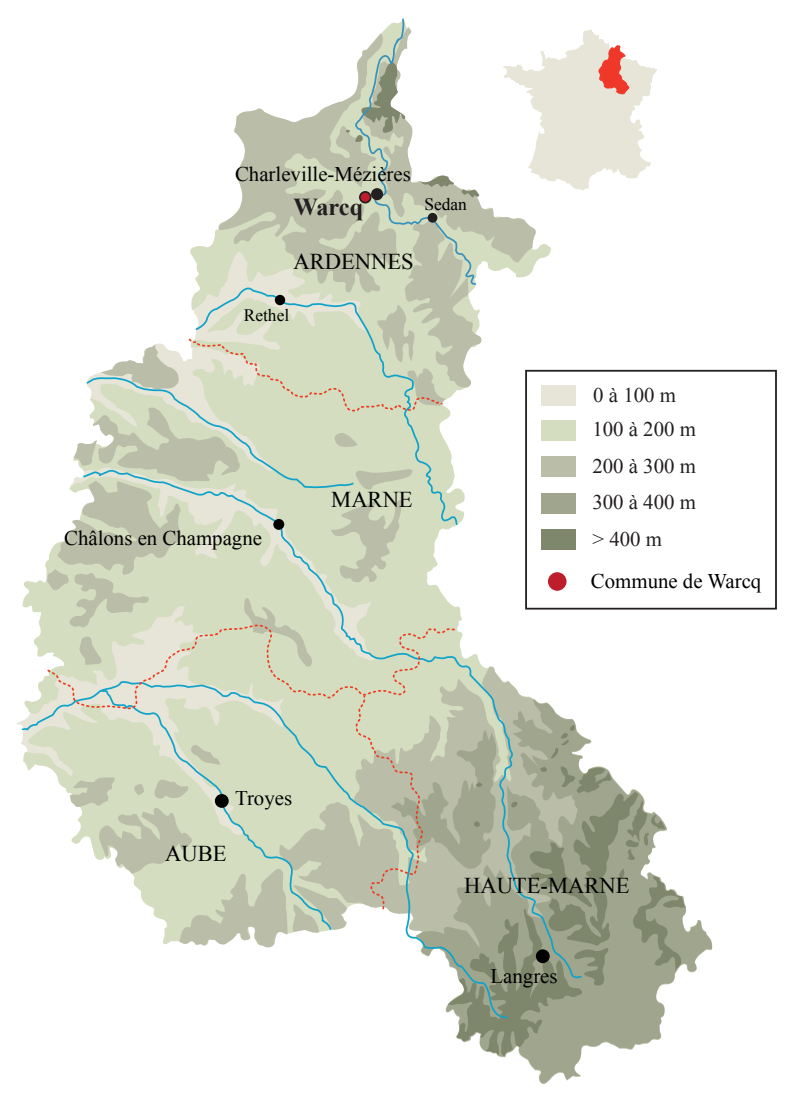

qui forment désormais un seul ensemble, sont reliés depuis peu à deux emprises linéaires, à savoir la prolongation de l'autoroute 34 vers la Belgique (A304 : branche ouest $d u$ « $Y »$ ardennais), entre la commune de La Francheville et celles de Rocroi / Gué d'Hossus, et le barreau de raccordement qui contournera Charleville-Mézières sur son côté ouest entre ce fuseau autoroutier et la RN43.

Le tracé de la future autoroute, qui se développera sur une longueur de $31 \mathrm{~km}$, a été intégralement sondé. Sur les 510 hectares étudiés au total, 140 I'ont été par les agents du conseil départemental des Ardennes (CD 08). Les résultats de ces opérations ont motivé la prescription de 11 fouilles (voir les tableaux ci-dessous). Celles-ci ont été réalisées entre 2011 et 2014. Cinq d'entre elles concernent la seule commune de Warcq. Le projet du barreau de raccordement porte quant à lui sur un total de 15 hectares, répartis sur une longueur de 3,3 km. Cette emprise a été sondée en quatre temps par les archéologues du CD 08, entre 2014 et 2016. Ces interventions ont débouché sur la réalisation de deux fouilles entre 2015 et 2017, chacune sur le territoire de Warcq.

\section{PRÉSENTATION GÉOGRAPHIQUE ET GÉOLOGIQUE}

La commune de Warcq est séparée de celle de Charleville-Mézières par un grand méandre de la Meuse. Le village actuel, qui s'est développé à l'emplacement d'une motte castrale attestée dès le $x^{e}$ siècle, se trouve à $3 \mathrm{~km}$ au sud-ouest de la préfecture des Ardennes. Il est implanté sur un sol très humide à la confluence du fleuve et des rivières Sormonne et This. Ce territoire s'inscrit entre les Crêtes Préardennaises, au sud, et le massif primaire, au nord. Du point de vue géologique, le sous-sol présent dans la partie septentrionale de la commune comprend deux entités principales appartenant au Lias (Jurassique inférieur) : les calcaires de Romery (Sinémurien moyen), qui se caractérisent par une alternance de calcaires gréseux de 0,2 à $0,8 \mathrm{~m}$ d'épaisseur et d'interbancs sableux

[1] CARTRON 2011a, p. 91-100. 


\begin{tabular}{|c|c|c|c|c|c|}
\hline Commune & Lieu-dit & $\begin{array}{l}\text { Responsable de } \\
\text { l'opération }\end{array}$ & Opérateur & $\begin{array}{c}\text { Année de } \\
\text { l'intervention }\end{array}$ & Principaux résultats \\
\hline Warcq & « La Haute Praële » & F. Charlier & $\begin{array}{c}\text { France } \\
\text { Archéologie }\end{array}$ & 2012 & Établissement rural antique \\
\hline Warcq & "Bois de Charnois" & B. Roseau & CD 08 & 2012 & $\begin{array}{c}\text { Installation du Bronze final et du } \\
\text { Hallstatt / Voie antique }\end{array}$ \\
\hline Warcq & «RD $16 »$ & G. Cartron & CD 08 & $2012 / 2013$ & $\begin{array}{c}\text { Établissement rural de La Tène et } \\
\text { de l'époque romaine }\end{array}$ \\
\hline Warcq & "La Sauce » & B. Roseau & CD 08 & 2013 & Établissement rural antique \\
\hline Warcq & "La Sauce » & B. Roseau & CD 08 et Inrap & 2014 & Tombe à char de La Tène finale \\
\hline $\begin{array}{l}\text { Remilly-les- } \\
\text { Pothées }\end{array}$ & " La Culotte» & B. Souffy & Inrap & $2012 / 2013$ & $\begin{array}{l}\text { Site mésolithique / Habitat, aire } \\
\text { funéraire et sanctuaire antique }\end{array}$ \\
\hline $\begin{array}{l}\text { Remilly-les- } \\
\text { Pothées }\end{array}$ & "Le Mazy » & Y. Rabasté & Inrap & 2011 & $\begin{array}{l}\text { Installation du Hallstatt / } \\
\text { Établissement rural antique }\end{array}$ \\
\hline Murtin-et-Bogny & "Le Pré Sauvignon » & Y. Rabasté & Inrap & 2011 & $\begin{array}{l}\text { Ateliers de métallurgie du haut } \\
\text { Moyen Âge }\end{array}$ \\
\hline $\begin{array}{l}\text { Le-Châtelet-sur- } \\
\text { Sormonne }\end{array}$ & $\begin{array}{l}\text { "Le Tranliau, } \\
\text { zones } 1 \text { et } 2 \text { " }\end{array}$ & $\begin{array}{l}\text { B. Duchêne et } \\
\text { M. Feller }\end{array}$ & Inrap & $2013 / 2014$ & $\begin{array}{l}\text { Établissement rural et aire } \\
\text { funéraire antique / Habitat } \\
\text { carolingien }\end{array}$ \\
\hline $\begin{array}{l}\text { Le-Châtelet- } \\
\text { sur-Sormonne / } \\
\text { Sévigny-la-Forêt }\end{array}$ & "Forêt des Pothées " & H. Bocquillon & Inrap & 2011 & Tumuli du second âge du Fer \\
\hline
\end{tabular}

\begin{tabular}{|c|c|c|c|c|c|}
\hline Commune & Lieu-dit & $\begin{array}{c}\text { Responsable de } \\
\text { Popération }\end{array}$ & Opérateur & $\begin{array}{c}\text { Année de } \\
\text { P'intervention }\end{array}$ & Principaux résultats \\
\hline Warcq/Belval & "Gosseval » & G. Cartron & CD 08 & 2015 & $\begin{array}{c}\text { Habitat de la Tène finale/ } \\
\text { Établissement rural antique }\end{array}$ \\
\hline Warcq & $\begin{array}{c}\text { "Sous le Chemin } \\
\text { de Tournes » et } \\
\text { "Simonelle » }\end{array}$ & J. Bruyère & CD 08 & 2017 & Édifice thermal antique \\
\hline
\end{tabular}

Tableaux récapitulatifs des fouilles menées dans le département des Ardennes préalablement aux deux principaux projets routiers (travaux de l'A304 en haut, ceux du barreau de raccordement en dessous)

d'épaisseur variable et, en-dessous, les marnes dites « de Warcq » (Sinémurien inférieur), formées de bancs de calcaires argileux dont l'épaisseur varie généralement entre 0,1 et $0,4 \mathrm{~m}$ et d'interbancs marno-silteux d'épaisseur sensiblement égale [2]. Dans la partie sud de la commune, occupée en grande partie par le bois de Charnois, les terrains affleurant sont des marnes bleues du Pliensbachien. L'étage, également daté du Lias, est défini comme des marnes franches sableuses et micacées. Ce relief vallonné, qui présente en certains endroits des pentes assez fortes, est entaillé par les vallées du This et de la Sormonne et, près de la limite sud de la commune, par celle des Rejets. L'étendue de ces vallées et l'importance de ces cours d'eau ont conduit à l'apparition d'une masse alluvionnaire importante (alluvions récentes et anciennes). En dehors de l'agglomération, la plupart des terrains

[2] WATERLOT et alii 1960.

[3] Cf. par exemple NiCOLAS 2011, p. 223-234, mais aussi les derniers rapports concernant les opérations archéologiques réalisées à cet endroit (LAURELUT 2012 à Montcy-Saint-Pierre et LAURELUT 2003 sur le plateau de Berthaucourt). sont aujourd'hui occupés par des cultures ou, lorsque la pente est trop marquée ou le sol particulièrement humide, par des bois et des pâtures.

\section{L'ÉTAT DE LA DOCUMENTATION ANCIENNE}

Les comptes rendus de découvertes, parfois fort anciens, laissaient déjà entrevoir la richesse archéologique de ce secteur, situé à environ $6 \mathrm{~km}$ à l'ouest des deux grandes zones d'habitat antiques - denses et structurées - connues sur le territoire de CharlevilleMézières (quartier de Montcy-Saint-Pierre et plateau de Berthaucourt) [3]. M.-F.-A. Duvivier, conservateur des antiquités du département des Ardennes et membre de la Société des antiquaires de France au cours de la première moitié du XIX $X^{e}$ siècle, est l'auteur de plusieurs documents manuscrits consacrés aux vestiges archéologiques de Warcq. Il y consigne notamment I'existence de ruines appartenant à un pont de pierre enjambant la Meuse, ou la découverte en 1795, plus au sud, d'un bloc de pierre portant une épitaphe d'époque romaine, à l'emplacement de la chapelle 
Figure 2

Les différentes occupations attestées sur le territoire de Warcq.

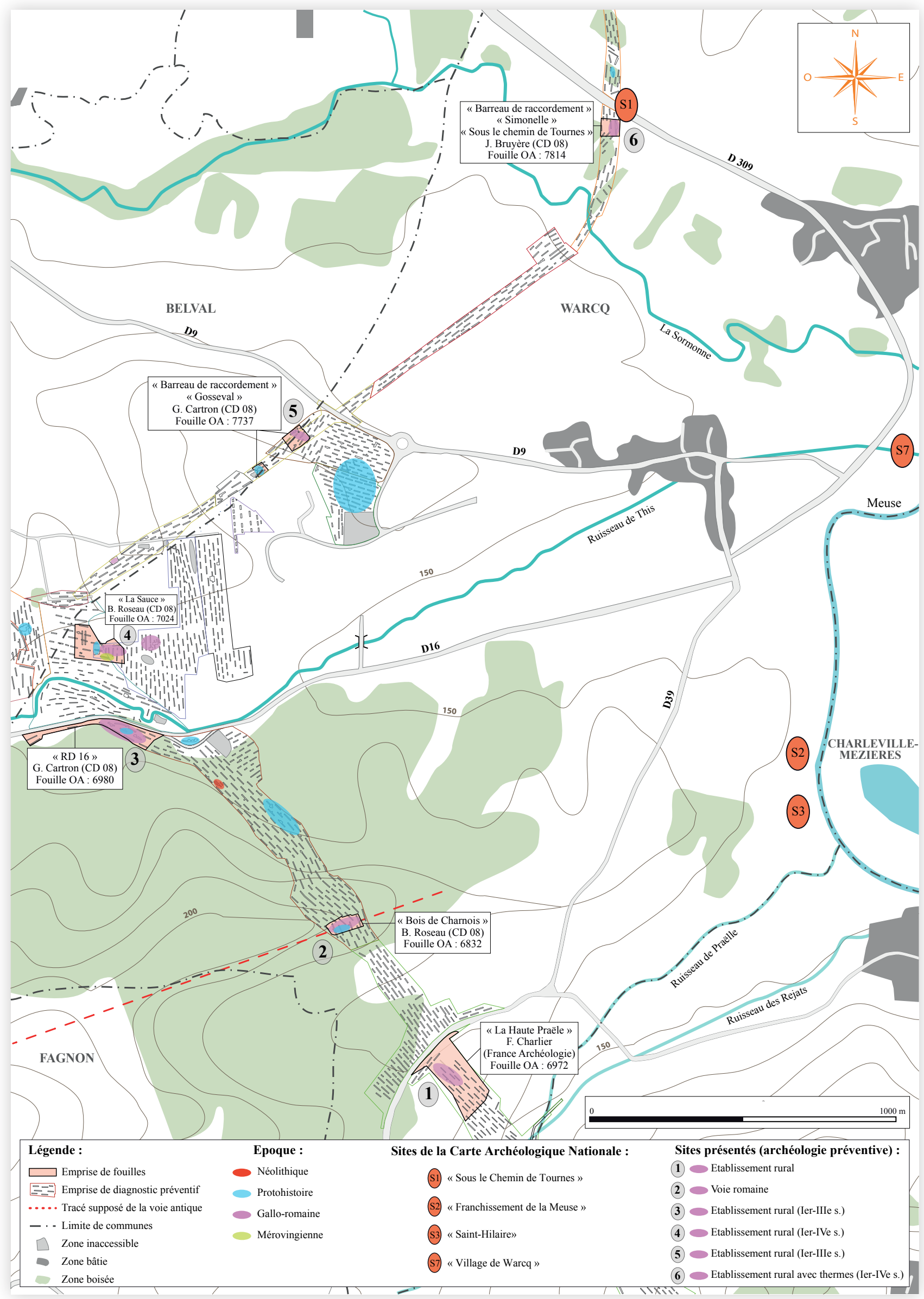


de Saint-Hilaire, au bord de la Meuse (CIL XIII, $n \circ 3453)$. Cet auteur signale également la mise au jour, dans les années 1760, d'un sarcophage en plomb contenant des ossements et d'une monnaie romaine au lieu-dit « La Fontaine », immédiatement au nord du village, ainsi que celle d'un bloc sculpté avec personnages en 1824, au centre du bourg [4]. Citons également, entre autres trouvailles, celles de structures maçonnées et de tessons de céramique antique réalisées dans les années 1970 et 1980 par un archéologue bénévole, J.P. Lémant, notamment dans la partie nord de la commune, au lieu-dit «Sous le Chemin de Tournes », ou à «SaintHilaire », probablement à proximité du passage d'une voie antique [5].

\section{L'APPORT DES OPÉRATIONS D'ARCHÉOLOGIE PRÉVENTIVE}

Si ces données, souvent sommaires, laissaient percevoir l'existence d'une occupation antique d'une certaine importance, elles ne permettaient pas de la caractériser. Les informations fournies par les opérations d'archéologie préventive organisées récemment à cet endroit renouvellent profondément nos connaissances sur l'implantation humaine dans ce secteur et permettent de replacer ces découvertes dans un contexte plus général. Il paraît intéressant de noter que la très grande majorité des sites désormais connus à Warcq - difficilement détectables par prospection aérienne, car bien souvent profondément enfouis ou matérialisés par des vestiges très ténus, certains se trouvant par ailleurs en milieu boisé - étaient inconnus avant la réalisation de sondages. Ce ne sont pas moins de 34 sites ou indices de sites qui ont été mis au jour au cours de ces interventions (fig. 2). Les premières traces de l'implantation humaine à Warcq appartiennent au Néolithique. Il s'agit de rares fosses contenant des tessons de céramique et des artefacts lithiques [6] ou - cas plus fréquent - de lots de silex taillés piégés dans le comblement de structures antiques. De nombreux vestiges révèlent par ailleurs une occupation particulièrement dense de ce secteur durant la Protohistoire. Une tombe à char inviolée a par exemple été fouillée en 2014 à quelques dizaines de mètres au nord du cours actuel du This, au lieudit «La Sauce » [7]. Sa vaste chambre était pourvue d'un coffrage en bois. Le défunt, inhumé avec un char d'apparat, était accompagné de quatre chevaux. Le riche mobilier recueilli dans cette tombe peut être daté du II ${ }^{\mathrm{e}}$ siècle av. J.C. Plus au nord, sur les pentes d'un vallon sec («La Haie de Gosseval »), une aire funéraire composée d'enclos fossoyés circulaires et de simples inhumations en pleine terre a été découverte au cours de deux diagnostics [8]. Les inhumations pourraient appartenir à La Tène A. Plusieurs zones d'habitat, dont la chronologie précise reste malheureusement difficile à fixer, ont en outre été mises en évidence grâce à divers sondages réalisés dans la commune, notamment aux lieux-dits « Jonet », « Le Pré Fossily » et, plus au sud, dans le bois de Charnois. Nous disposons en revanche de peu de données archéologiques concernant I'occupation après la période romaine. On notera toutefois la découverte en 2011, à la limite entre les communes de Warcq et de Belval («Le Pré Fossily »), d'un aménagement en bois conservé près du cours actuel du This [9]. Observée sur une longueur totale de $7,8 \mathrm{~m}$, cette structure est composée de poutres et de planches en chêne et en aulne. Les prélèvements effectués dans ces bois permettent de placer la réalisation de ce dispositif autour de 543 ap. J.C., avec une possible réfection vers 577 .

\section{LES SITES ANTIQUES FOUILLÉS}

12 sites attribuables à l'époque romaine, découverts fortuitement ou à l'occasion de diagnostics archéologiques, sont désormais recensés sur cette commune. Nous verrons qu'il s'agit en très grande partie de structures d'habitat, les aires funéraires devant probablement être cherchées le long de la voie antique présente dans la partie sud de ce territoire ou, légèrement plus au nord, près du village actuel.

Nous nous proposons de présenter ici chacune des zones d'occupation antique fouillées à Warcq, tout d'abord en évoquant leur situation topographique, puis en décrivant les structures les plus importantes, tout en rappelant les principales phases chronologiques représentées. En effet, bien que la quasi-totalité des prescriptions de fouilles émises sur cette commune repose sur la découverte de vestiges d'époque romaine, les opérations ont montré que ces gisements étaient pour la plupart diachroniques. Le cadre de notre présentation est ici géographique,

[4] Duvivier 1821 ; Duvivier 1825.

[7] ROSEAU à paraître.

[5] FREZOULS 1977, p. 397 ; LEMANT 1984, p. 16-17.

[6] CARTRON 2011b.

[8] CARTRON 2010 ; GUCKER 2010.

[9] CARTRON \& Duvauchelle 2015. 


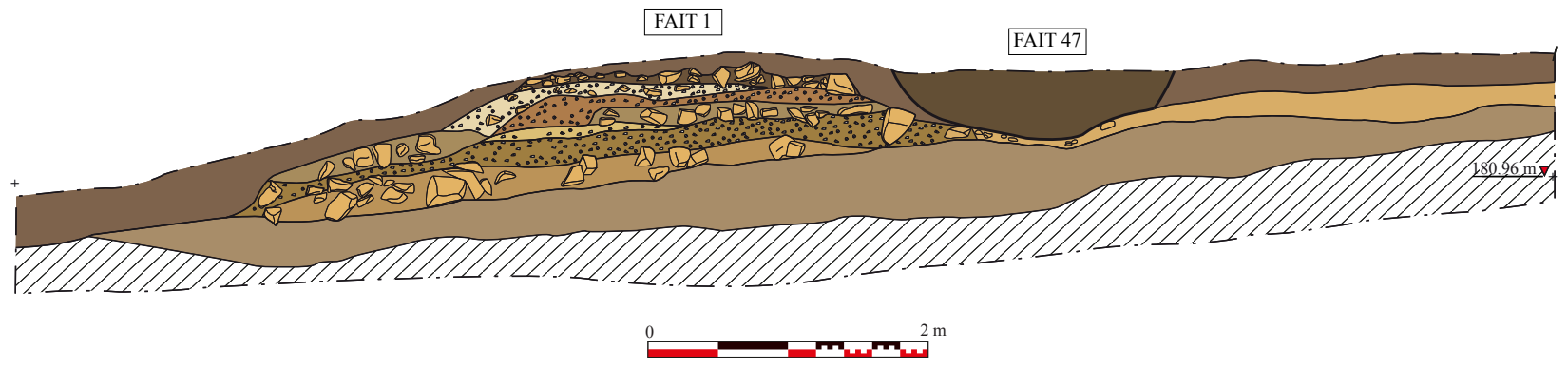

Figure 3 : coupe transversale de la voie étudiée dans le bois de Charnois (C) CD 08).

avec une progression du sud vers le nord. Celui-ci respecte par ailleurs la chronologie des interventions archéologiques.

\section{« LA HAUTE PRAËLE »}

Parmi les sites fouillés à Warcq, celui localisé au lieu-dit « La Haute Praële » est le seul à ne pas avoir été étudié par la cellule archéologique du CD 08. Le degré d'informations dont nous disposons est ici très faible, l'opérateur en charge de cette intervention ayant cessé d'exister avant que le rapport de fouille n'ait été rendu au service régional de l'Archéologie (SRA). L'opération concernait ici un établissement implanté sur le versant nord de la vallée des Rejets, près de la limite méridionale de la commune. Le plus grand des deux bâtiments dégagés était du type à galerie et pavillons d'angle. Il était ouvert vers le sud. L'un de ses pavillons d'angle était occupé par une cave maçonnée très bien conservée, dotée de niches voûtées. Cet espace de stockage, accessible par un escalier en pierre, a notamment fourni une colonnette préservée dans sa quasi-intégralité, munie d'un chapiteau, et un fragment d'une colonne reposant peut-être autrefois sur le mur-bahut de la galerie d'entrée. L'abandon de cet édifice est marqué par une série d'inhumations dont la datation reste à fixer.

\section{«BOIS DE CHARNOIS 》}

À 500 m au nord du site de la « La Haute Praële », un tronçon d'une chaussée présumée romaine a été fouillé à l'été 2012 dans le bois de Charnois, sur un terrain de $4000 \mathrm{~m}^{2}$. Le tracé de cette voie est relativement bien connu entre Reims et le point de franchissement de la Meuse situé à Warcq, légèrement en amont du village, au lieu-dit «SaintHilaire » [10]. Il est communément admis que ce tronçon appartiendrait à l'itinéraire impérial ReimsCologne, représenté sur la Table de Peutinger. Cette hypothèse doit cependant être considérée avec prudence, les données archéologiques disponibles sur ce sujet étant rares, de fortes incertitudes pesant par exemple sur son tracé dans le massif primaire de l'Ardenne [11].

Ce tronçon orienté nord-est/sud-ouest était parfaitement identifiable dans le paysage avant la fouille. Celui-ci affleurait en effet sous un niveau forestier atteignant au maximum 0,4 m d'épaisseur. Cette portion de chaussée se présentait sous la forme d'une structure rectiligne à section convexe. Elle formait ainsi une sorte de merlon à flanc de colline, descendant doucement vers le nord-est. Au-delà de la partie fouillée, le tracé de cette voie peut être suivi encore aujourd'hui sur quelques centaines de mètres au moins, que ce soit plus à l'ouest, en direction de Reims, mais aussi dans la direction opposée, vers le lieu-dit « Saint-Hilaire». Assez bien conservée globalement dans ce bois, bien que d'importantes dégradations d'origine anthropique ou naturelle aient été observées en certains endroits, cette portion de voie servait encore récemment de chemin de débardage.

La voie a été mise au jour sur une longueur totale de $110 \mathrm{~m}$, soit une superficie d'environ $657 \mathrm{~m}^{2}$. Sa largeur conservée était comprise, à la base, entre 4,63 et $6,03 \mathrm{~m}$, son épaisseur globale entre $0,75 \mathrm{~m}$ et $0,85 \mathrm{~m}$. Cette structure était composée de trois radiers superposés alternant avec des couches de sable et de gravier épaisses de 0,1 à 0,2 m (fig. 3). Les radiers, dont l'épaisseur oscillait entre 0,15 et 0,2 m, étaient formés de blocs de calcaire jaune à peine dégrossis. Deux alignements de blocs de calcaire gréseux jaune formaient les bordures nord et sud, larges chacune de $0,6 \mathrm{~m}$. Selon l'étude réalisée par G. Fronteaux et S. Laratte (Université de Reims, Gegenaa), les divers matériaux utilisés ici témoignent de multiples sources locales d'approvisionnement. Les gisements situés

[10] Pour une synthèse sur le tracé de la voie ReimsCologne dans le département des Ardennes, on se réfèrera à NiCOLAS 2011, p. 120.

[11] À propos de la traversée du massif ardennais de la voie Reims-Cologne, voir les hypothèses formulées dans Corbiau 1983 et Corbiau \& YANTE 2010. 
à moins de $5 \mathrm{~km}$ de l'axe routier semblent avoir été privilégiés. Il est d'ailleurs possible que certains matériaux aient pu faire l'objet d'un ramassage de « plein champ ». Notons enfin que le radier de fondation, ou statumen, scellait un paléosol. Préservé de l'érosion grâce à la présence de la voie, ce niveau recelait un ensemble de fosses datables du Bronze final et de La Tène ancienne. L'étude de ce sol ancien a montré un hiatus entre les deux occupations.

Seuls quelques éléments anciens de mobilier métallique ont été retrouvés au cours de cette opération. Ils ne permettent pas de préciser la date de construction et de mise en service de la chaussée, habituellement fixées au $\mathrm{I}^{\text {er }}$ siècle de notre ère. Il n'a pas non plus été possible de déterminer sa date d'abandon, placée par la plupart des chercheurs vers la fin du Ive siècle, bien qu'il existe des témoignages d'une utilisation au cours du Moyen Âge.
«RD 16 》

L'emprise fouillée le long de la route départementale $n^{\circ} 16$, qui couvrait un hectare, se trouve à environ $800 \mathrm{~m}$ au nord de la voie antique. Ce site est implanté sur le rebord en légère pente d'une terrasse alluviale, à une quarantaine de mètres des rives actuelles du This (fig. 4), modeste cours d'eau se jetant $2,5 \mathrm{~km}$ plus à l'est dans la Sormonne, au nord du village, juste avant la confluence avec la Meuse. Localisés en lisière d'un bois, dans un milieu relativement peu modifié par les activités humaines, les vestiges antiques présentent ici une puissance stratigraphique rarement rencontrée dans le département des Ardennes.

Si une première occupation durant le Néolithique final est supposée grâce à la découverte, en position secondaire, d'une série de silex taillés, les structures les plus anciennes correspondent sans doute à un établissement

Figure 4

Les constructions fouillées près de la «RD 16 » et, plus au nord, celles de « La Sauce ».

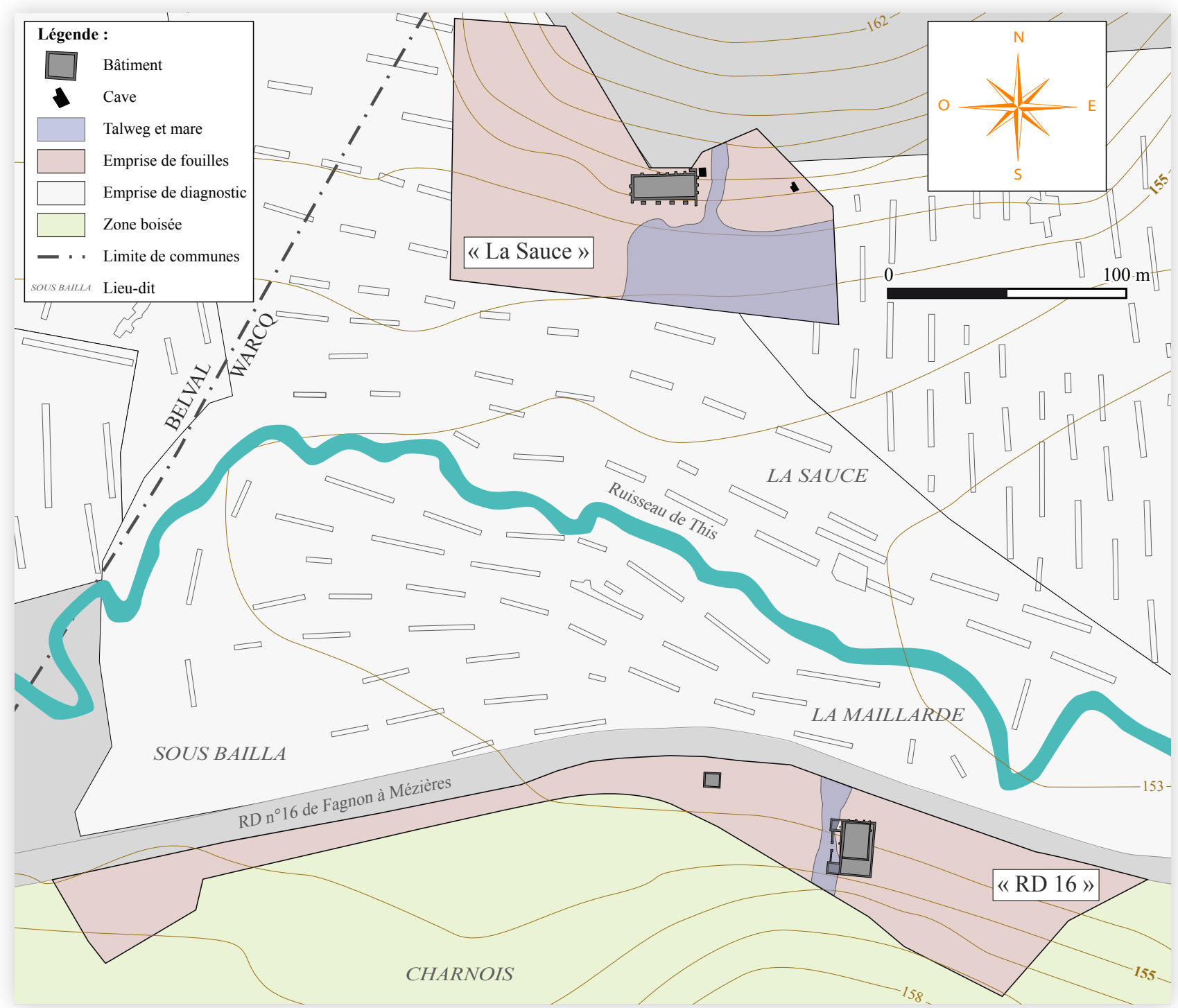


rural laténien matérialisé par au moins deux bâtiments en matériaux périssables. Le bâti du plus grand n'est pas restituable, car fortement altéré par les aménagements postérieurs. Le second édifice, situé $75 \mathrm{~m}$ plus à l'ouest, était de type à pans coupés et atteignait une superficie interne proche de $70 \mathrm{~m}^{2}$. Cet établissement pourrait avoir été créé, d'après les analyses au radiocarbone, au IVe ou au III $^{\mathrm{e}}$ siècle avant notre ère.

L'occupation antique qui lui succède, probablement après un hiatus chronologique qu'il n'a pas été possible de définir, présente trois états d'après l'étude du matériel céramique. Le premier correspond à une série de structures en creux comblées au cours de la première moitié du I $^{\text {er }}$ siècle de notre ère. L'état suivant est notamment marqué par la création de deux bâtiments. Le plus grand était construit entièrement « en dur » à I'emplacement du principal édifice laténien, suivant une orientation très légèrement divergente. Cette construction pourvue de contreforts extérieurs sur trois côtés

\section{Figure 5}

Une partie du dispositif hydraulique découvert à proximité immédiate du bâtiment principal de la « RD $16 \gg$ (C) CD 08).

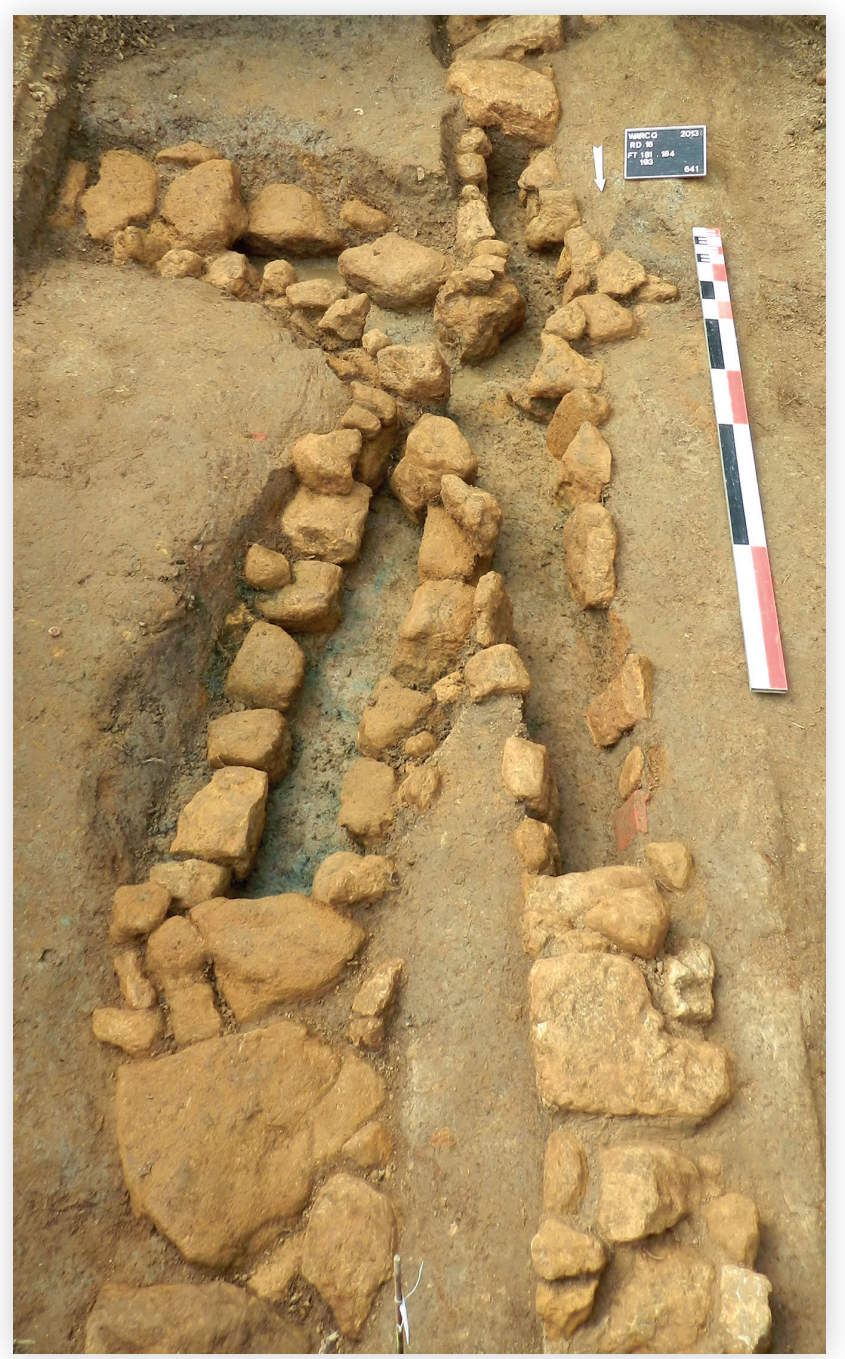

$\mathrm{s}^{\prime}$ inscrit dans un rectangle long de 16,2 m et large de $12,3 \mathrm{~m}$. Elle est établie parallèlement à la pente naturelle du terrain, le long d'un talweg inscrit dans le toit du substrat, large de 6 à $12 \mathrm{~m}$ à l'ouverture et profond de 1,1 à 3,3 m. Le second bâtiment, de taille bien plus modeste, avait été élevé une cinquantaine de mètres plus à l'ouest. Parmi les structures aménagées en son sein, une fosse se distingue par la présence de rouelles en plomb et en alliage cuivreux dans son comblement, nous invitant à nous interroger sur la fonction de cette construction de plan sensiblement carré. Une expansion du domaine est tangible durant l'état suivant, c'est-à-dire à partir de la fin du $\mathrm{I}^{\mathrm{er}}$ siècle ap. J.C. ou du début du siècle suivant. La nouvelle bâtisse témoigne d'une certaine recherche de monumentalité, puisqu'elle comporte une salle de 21,9 m de long sur $12 \mathrm{~m}$ de large, précédée par une galerie de façade et deux pavillons d'angle saillants. Elle présente une surface habitable totale d'environ $340 \mathrm{~m}^{2}$, soit le double de l'édifice à contreforts de l'état précédent. Cette extension du bâti vers l'ouest a entraîné une importante modification de la topographie, I'ancien talweg devant être préalablement comblé sur toute sa hauteur, de manière à niveler le terrain. Ce travail n'a pas été effectué d'un seul jet, puisque les constructeurs ont manifestement pris la précaution d'assainir ce secteur en installant plusieurs drains à différents niveaux de remplissage de la dépression (fig. 5). Un chemin empierré a ensuite été créé au-dessus de cette zone colmatée. Reconnu sur 38 m de long devant la façade de l'édifice, celui-ci effectue dans la partie basse de l'emprise un coude vers l'est, s'étirant sur $47 \mathrm{~m}$ de long parallèlement au petit côté nord de la bâtisse. Le domaine est bordé à cette époque par des séries de poteaux et de fossés matérialisant une clôture de plan trapézoïdal épousant au nord les courbes de niveau. Mise au jour sur une surface d'environ $3500 \mathrm{~m}^{2}$, elle s'étend manifestement plus au sud, en dehors des limites de la fouille, en direction du bois. Enfin, un four de potier découvert près de la limite orientale de cet établissement, immédiatement en dehors de la clôture, pourrait être en lien avec cet état du site. L'abandon de l'ensemble des structures peut être fixé à la fin du III $^{\mathrm{e}}$ siècle de notre ère ou au début du siècle suivant.

\section{« LA SAUCE 》}

De l'autre côté du ruisseau de This, à environ $200 \mathrm{~m}$ au nord du site de la «RD 16 » (fig. 4), une autre implantation gallo-romaine a fait l'objet d'une fouille dans le cadre du projet autoroutier. Les vestiges se trouvent en bordure d'une zone humide, non loin du cours d'eau. Ils sont implantés sur une déclivité nord-sud 
marquée par une rupture de pente relativement nette, dont la présence a probablement facilité l'accumulation de colluvions. Ces couches, dont la puissance cumulée pouvait atteindre jusqu'à $2,5 \mathrm{~m}$, ont formé une gangue protectrice au-dessus des structures. Aucun élément visible dans le paysage ne permettait ainsi de soupçonner leur présence avant les travaux. Malgré tout, non seulement les vestiges mis en évidence sont particulièrement nombreux, puisque pas moins de 500 structures en creux ont été répertoriées au total, mais cet habitat occupe une surface bien plus vaste que ne le laissaient supposer les résultats des diagnostics. Ainsi, malgré une étendue de $8000 \mathrm{~m}^{2}$, l'emprise de fouille n'a pas permis d'appréhender l'extension totale du site, ni même une seule de ses limites.

D'époque gallo-romaine, l'occupation principale s'articule autour d'une grande bâtisse rectangulaire (28 $\mathrm{x}$ $11 \mathrm{~m}$ ) orientée est-ouest, construite en moellons de calcaire jaune et couverte d'ardoises. Des contreforts sont espacés tous les $3 \mathrm{~m}$ le long de ses murs nord, est et ouest. Sur le côté sud, un avant-toit est soutenu par une série de piliers en matière périssable espacés de 5,7 m. La surface interne, indivise, est de $270 \mathrm{~m}^{2}$. Une cave découverte immédiatement à l'est de cette construction $\left(10 \mathrm{~m}^{2}\right)$, dont les parois étaient autrefois doublées par des planches de bois (fig. 6), possède

Figure 6

Une cave fouillée à « La Sauce », abandonnée dans le dernier tiers du III $^{\mathrm{e}}$ siècle ap. J.C. ou au début du siècle suivant (C) CD 08).

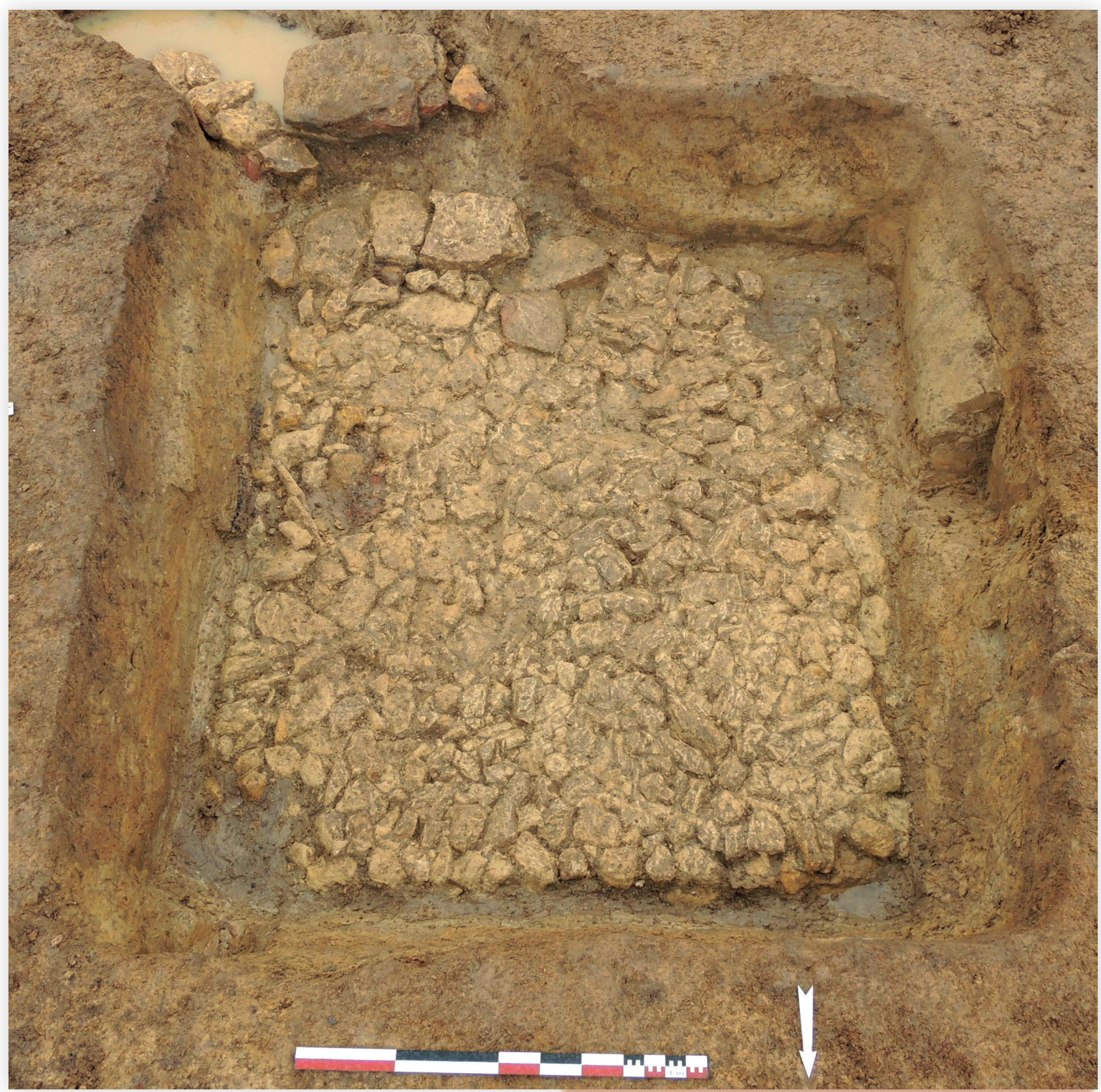


un système de drainage permettant de rejeter l'eau dans une dépression sans doute naturelle bordant I'édifice. Cette eau alimentait ensuite un étang situé au sud-est du bâtiment. L'ensemble constitue probablement une partie de la pars rustica d'un grand domaine. Tous les indices recueillis dans le cadre de I'étude carpologique, mais aussi grâce au mobilier métallique ou lithique, indiquent une activité agricole et artisanale prédominante au moment de l'abandon du bâtiment. D'un point de vue chronologique, l'étude de la céramique conjuguée à celle de la stratigraphie permettent de dater son effondrement et son abandon à la fin du III $I^{\mathrm{e}}$ ou au début du IV ${ }^{\mathrm{e}}$ siècle de notre ère. Des indices plus ténus mis au jour à l'est et à l'ouest de ce noyau principal sont interprétés comme les ves- tiges de bâtiments sur poteaux d'époques diverses. En effet, trois autres phases d'occupation gallo-romaine ont pu être mises en évidence grâce au matériel archéologique, en particulier la céramique. Une première phase, attestée par de rares éléments de mobilier, date de la période julio-claudienne, et plus précisément du début du $\mathrm{I}^{\mathrm{er}}$ siècle ap. J.-C. La deuxième, documentée par quelques structures creusées à I'ouest du bâtiment agricole, peut être placée à la charnière des $\mathrm{I}^{\mathrm{er}}$ et $\mathrm{II}^{\mathrm{e}}$ siècles. Enfin, une troisième phase a été identifiée près de l'extrémité orientale de l'emprise, dans une probable cave ayant fourni du matériel du deuxième tiers du IV ${ }^{\mathrm{e}}$ siècle. Les matériaux de construction retrouvés dans ce secteur (tubulures, briques, tuiles, etc.) invitent également à restituer

Figure 7 : vue de la cave de «Gosseval » et de son dispositif hydraulique interne (C) CD 08).

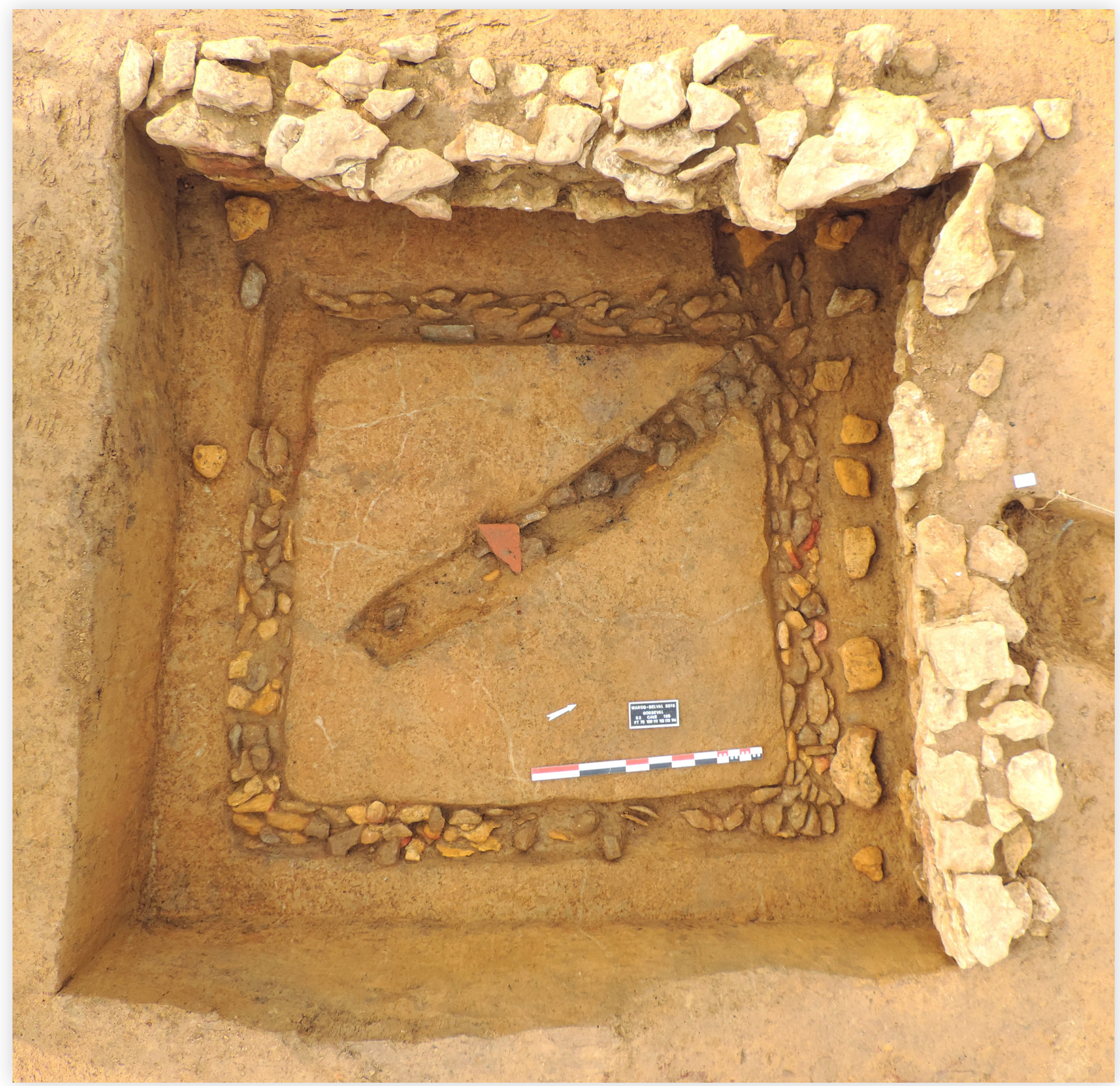


d'autres structures maçonnées en dehors du tracé autoroutier.

Une occupation mérovingienne est en outre attestée par du mobilier et des sépultures. Ces vestiges, qui peuvent être datés entre la seconde moitié du $\mathrm{VI}^{\mathrm{e}}$ et la première moitié du VIII ${ }^{\mathrm{e}}$ siècle, occupent principalement la partie occidentale de l'emprise fouillée. Deux tombes coupaient toutefois le mur sud et les couches d'abandon du bâtiment agricole gallo-romain. Une autre phase est représentée par une exceptionnelle tombe à char de La Tène C2-D1, dont aucun élément ne laissait supposer la présence. Celle-ci a été découverte lors du démontage des couches d'effondrement de l'édifice gallo-romain.

\section{« GOSSEVAL 》}

Si une partie de la fouille organisée au lieu-dit « Gosseval » sur un terrain de $5300 \mathrm{~m}^{2}$ concernait une zone d'habitat implantée près du fond d'un vallon entre La Tène B1 et La Tène D2, les structures trouvées sur la partie haute de cette même pente appartenaient à l'époque romaine. Une petite cave aménagée près du sommet de la crête était pourvue d'un ingénieux dispositif hydraulique (fig. 7), permettant de rejeter les eaux superflues à l'extérieur de la structure de stockage dans un drain en pierres long de $54 \mathrm{~m}$. Parmi les nombreux éléments témoignant du violent incendie survenu dans cet espace, on signalera la présence de plusieurs planches carbonisées à la base de la fosse d'implantation, qui appartenaient sans doute pour certaines à un plancher, pour d'autres au revêtement des parois. Les six échantillons de bois confiés pour étude à W. Tegel (laboratoire DendroNet, à Bohlingen) ne possédaient pas suffisamment de cernes pour une datation dendrochronologique. L'analyse des essences a en revanche montré une cohabitation entre un élément très résistant aussi bien en milieu sec qu'en milieu humide (chêne) et deux autres aux potentiels technologiques assez faibles (hêtre et saule). Ces résultats sont assez surprenants, dans la mesure où les autres sites antiques de ce département n'avaient fourni, jusqu'à présent, que des bois d'œuvre en chêne. À une dizaine de mètres de là ont été trouvés une mare occupant une surface au sol de $253 \mathrm{~m}^{2}$ (dimensions à son niveau d'apparition : $21 \times 15,5 \mathrm{~m}$ ), conservée sur $1 \mathrm{~m}$ de profondeur environ, et un cellier dont ne subsistait que la partie basse des parois. Cette mare et la cave auraient été abandonnées entre 260 et 290 ap. J.C. d'après le matériel céramique trouvé dans leur remplissage. Une seconde mare, de dimensions bien inférieures $(11,70 \times 9,75 \mathrm{~m}$ au niveau de décapage, soit un peu plus de $90 \mathrm{~m}^{2}$ ), a été appréhendée en contrebas. Cette dépression, observée sur 1,3 m de hauteur, a révélé deux principales phases de comblement : les premières strates remonteraient à une période comprise entre le règne d'Auguste et celui de Néron, tandis que son scellement - matérialisé par un niveau de colluvionnement - contient du mobilier datable de la fin du $\mathrm{I}^{\mathrm{er}}$ siècle ap. J.C. et/ou du début du siècle suivant.

\section{« SOUS LE CHEMIN DE TOURNES 》 ET «SIMONELLE 》}

La fouille de Warcq «Simonelle » «Sous le chemin de Tourne » s'est déroulée au printemps 2017 sur une surface de $3400 \mathrm{~m}^{2}$. Outre trois structures en creux potentiellement datées de la période protohistorique, le décapage a permis de mettre au jour un ensemble de maçonneries de l'époque romaine sur près de $300 \mathrm{~m}^{2}$, dont l'état de conservation est exceptionnel. Cette construction est installée à environ $500 \mathrm{~m}$ au nord de la rivière La Sormonne, à une altitude de $150 \mathrm{~m}$ NGF. D'un point de vue géologique, le site se trouve à la jonction des alluvions anciennes et du Sinémurien supérieur. L'étude des vestiges, toujours en cours, fait état d'une occupation entre le $\mathrm{II}^{\mathrm{e}}$ et le $\mathrm{IV}^{\mathrm{e}}$ siècle de notre ère. L'organisation spatiale des vestiges évoque une partie des bâtiments d'une villa gallo-romaine (pars urbana). En effet, la fouille a permis de mettre en évidence un espace ouvert, une cave et des éléments de bains.

Quatre phases d'occupation peuvent être discernées, certaines matérialisées par d'importants réaménagements (fig. 8). Le premier état est caractérisé par un espace ouvert et une cave. L'espace ouvert, qui occupe la partie orientale de l'emprise, se développe en dehors de ce terrain. Sa superficie est supérieure à $50 \mathrm{~m}^{2}$. Cet aménagement est circonscrit par des murs larges de $1 \mathrm{~m}$, bâtis en parement et blocage. Accessible par une volée de quatre marches ouverte à l'est, la cave occupe une surface rectangulaire de $15,75 \mathrm{~m}^{2}$ (4,5 x 3,5 m hors œuvre). Les maçonneries, conservées sur près de $1,8 \mathrm{~m}$ de haut, offrent un parement en opus vittatum avec des joints lissés au fer. Les marches sont des monolithes calcaires d'une épaisseur moyenne de 0,3 $\mathrm{m}$. Les différents murs de la cave sont agrémentés de niches voûtées. Celui du nord est doté d'un soupirail.

La deuxième phase d'occupation voit la création de thermes constitués de cinq pièces, dont deux de service (fig. 9). Cet ensemble de forme allongée vient s'appuyer sur le mur occidental de la phase précédente. Les pièces de bains, toutes en enfilade, possèdent les mêmes dimensions, soit 3,3 $\mathrm{m}$ de long pour $2 \mathrm{~m}$ de large. Le frigidarium se distingue par l'ajout 

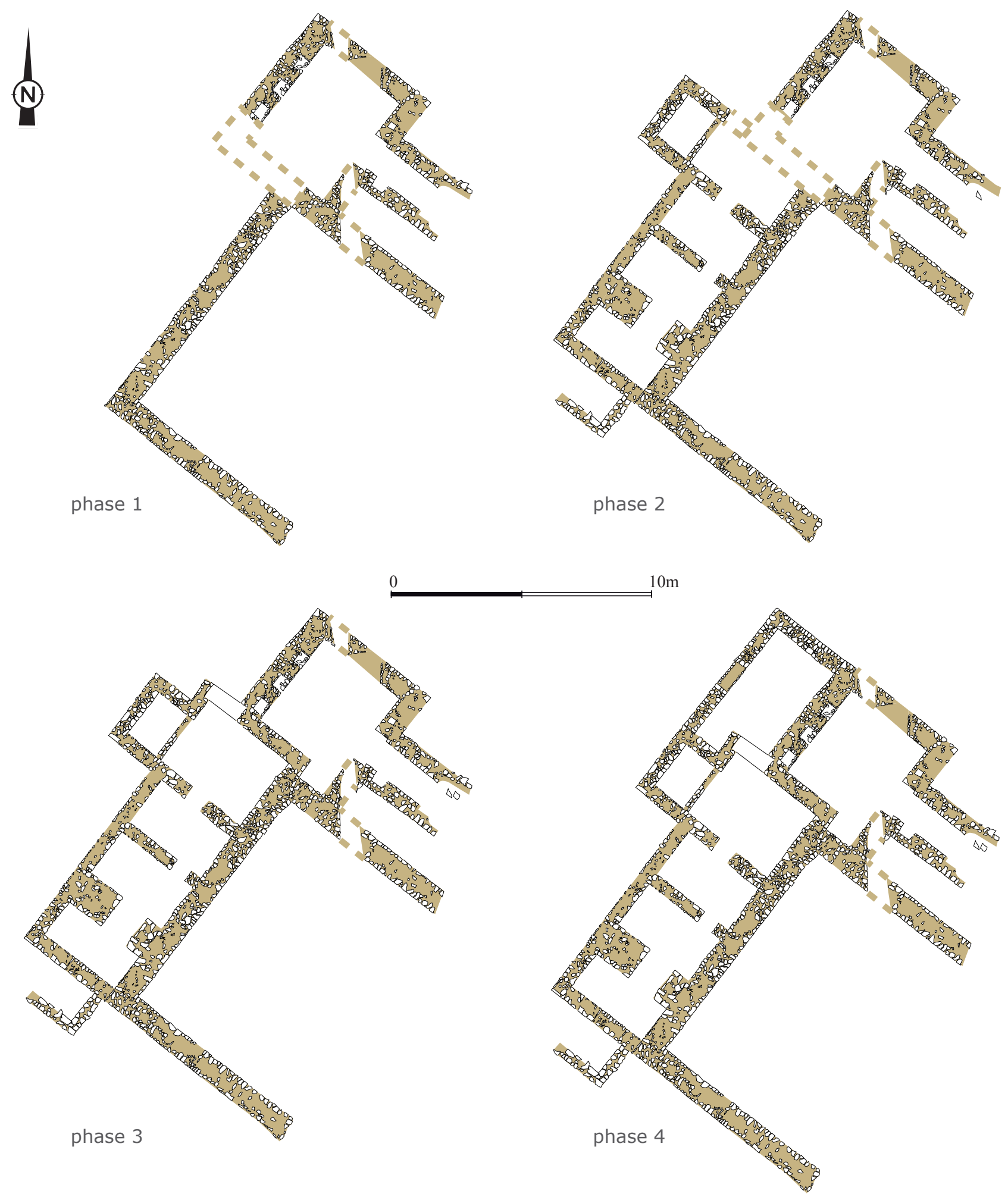

Figure 8 : les quatre états des thermes (@ CD 08).

d'une piscine mesurant $1,65 \mathrm{~m}$ de long pour $1,2 \mathrm{~m}$ de large. Le caldarium et le tepidarium, qui présentent un excellent niveau de conservation, sont bâtis sur des hypocaustes encore partiellement en place. La circulation de l'air entre la pièce chaude et la pièce froide s'effectue au moyen de deux passages ménagés dans le mur. Les tubulures sont disposées sur trois des quatre murs de chaque salle. Le mur occidental, non pourvu de tubuli, servait d'appui à un bassin en mortier de tuileau. La pièce chaude se singularise par la présence d'un second bassin. De petites dimensions $(1,3 \times 1 \mathrm{~m})$, celui-ci était construit directement sur le conduit du canal de chauffe. Le décor des salles pourvues de bains était composé pour l'essentiel d'éléments en marbre. Le système de chauffage était assuré par un praefurnium, petite pièce $(3,3 \times 1,5 \mathrm{~m})$ 


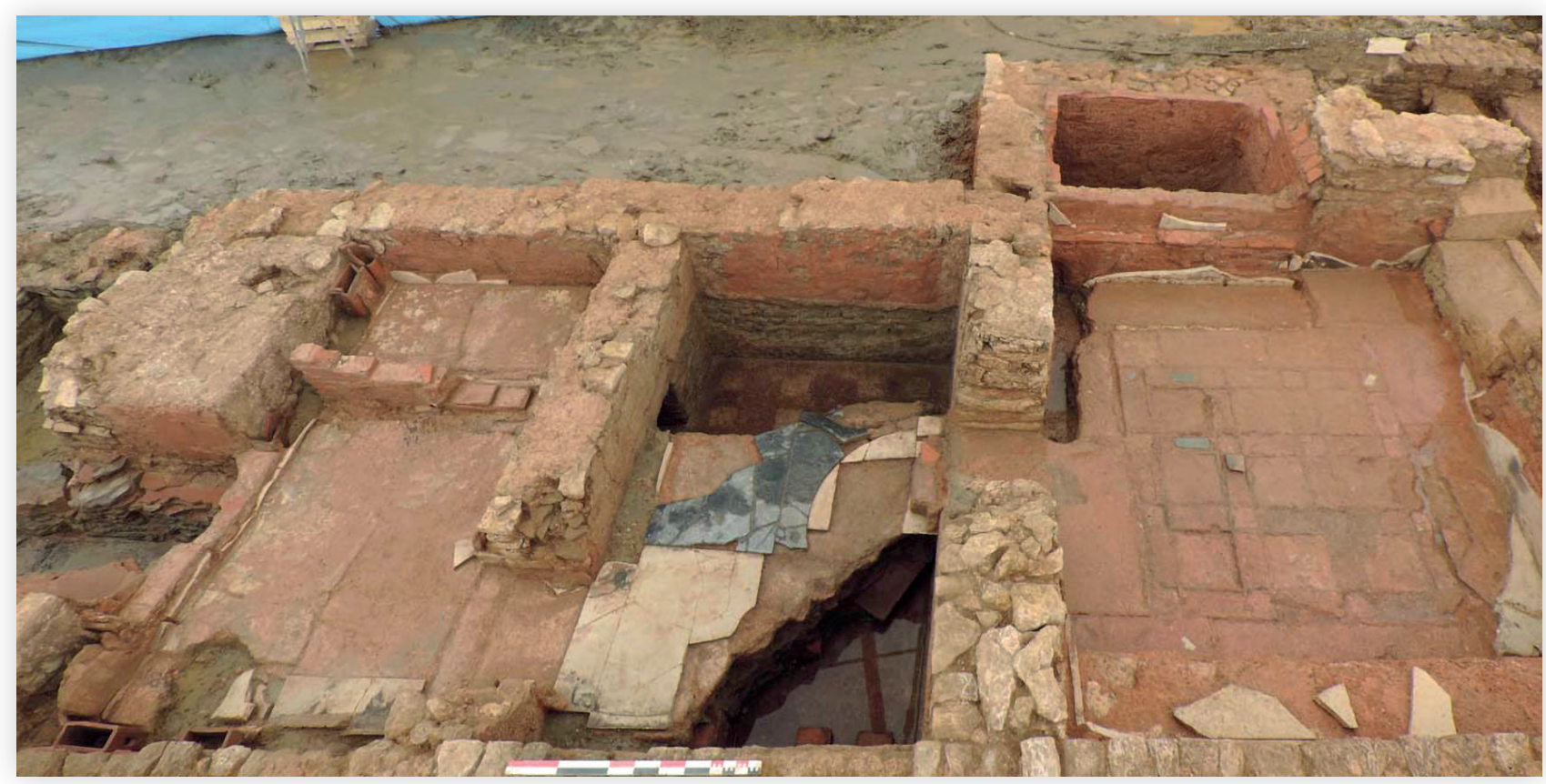

Figure 9 : Les trois pièces de bains des thermes. De gauche à doite : caldarium, tepidarium, frigidarium. (C) CD 08).

accueillant un canal permettant d'envoyer l'air chaud. Les maçonneries sont conservées à cet endroit sur quelques assises, tandis que le sol ne révèle aucune trace d'aménagement (terre battue ?). La deuxième pièce de service, située au sud du praefurnium, se compose d'un petit espace en forme de « $L \gg(2 x$ $1,5 \mathrm{~m}$ ). Elle devait vraisemblablement servir d'accès au personnel.

La troisième phase d'occupation du site correspond à I'agrandissement du frigidarium aux dépens de la cave. La pièce froide prend alors une forme rectangulaire de 3,8 $\mathrm{m}$ de long pour 2,9 $\mathrm{m}$ de large (sans compter la piscine adjacente). La partie orientale est composée d'une banquette se développant sur toute la longueur du mur. Le sol, particulièrement bien conservé, est en opus sectile.

Le dernier agencement au sein de ces thermes est marqué par l'apparition d'un apodyterium. Il reprend les murs de la cave et vient s'insérer en amont de la pièce froide. Le vestiaire se présente sous la forme $\mathrm{d}$ 'une pièce rectangulaire de $4 \mathrm{~m}$ de long pour $2,7 \mathrm{~m}$ de large (hors œuvre). L'entrée s'effectue par l'ouest du bâtiment. Si les niveaux de sol ne sont pas préservés, on observe néanmoins un niveau de préparation. Il s'agit d'une couche de béton de tuileau épaisse d'une dizaine de centimètres. Elle possède la particularité d'être coupée par quatre tranchées transversales de $0,3 \mathrm{~m}$ de large et 0,2 $\mathrm{m}$ de profondeur, peut-être des négatifs d'une structure en matériaux périssables (lambourdes de plancher ?).

La fondation de ces bains ne semble pas remon- ter à la phase initiale de construction du bâtiment résidentiel. Cet ensemble thermal, qui imposait vraisemblablement aux usagers un itinéraire rétrograde, vient former ce qui pourrait être l'aile occidentale de la villa urbana.

\section{PERSPECTIVES}

La zone de découverte, située près de la Meuse et parcourue par de multiples cours d'eau secondaires, est de taille assez restreinte. Une telle concentration de sites d'époque romaine à cet endroit ne laisse pas d'étonner (un tous les 10 hectares sondés environ). Elle est par exemple très largement supérieure à celles enregistrées récemment dans le cadre du projet Rurland, qui se propose d'examiner l'évolution du monde rural dans le quart nord-est de la Gaule romaine. Une densité de 3,1 sites antiques par $\mathrm{km}^{2} \mathrm{a}$ ainsi été calculée pour la Plaine de France (3\% de ce territoire de $625 \mathrm{~km}^{2}$ explorés par l'archéologie), et respectivement de 1,6 et 1,2 établissement par $\mathrm{km}^{2}$ pour la Bassée et la vallée de la Moselle (respectivement $7 \%$ étudiés d'une zone de $325 \mathrm{~km}^{2}$ et $6,5 \%$ d'une zone de $476 \mathrm{~km}^{2}$ ) [12]. Sur le plateau lorrain, entre Seille et Nied, la densité d'occupation dépasse 3 sites par $\mathrm{km}^{2}$, alors que sur le plateau de Haye,

[12] Bernigaud et alii 2016, p. 70. 
toujours en Lorraine (entre l'agglomération de Nancy et le nord de la commune de Neufchâteau), elle n'est que de 1,1 site par $\mathrm{km}^{2}$ dans ce secteur certes très boisé [13].

Le territoire de l'actuelle commune de Warcq a-t-il connu un mode d'occupation particulier, témoignant par exemple d'un effet de polarisation, ou faut-il plutôt y voir la conjoncture des recherches archéologiques menées ces dernières années dans ce secteur? Devant la difficulté de répondre à cette question, nous nous contenterons de souligner l'existence d'un vide documentaire à l'ouest de cette commune. Nous disposons également de peu d'informations archéologiques à propos du massif primaire, milieu très boisé et au sol schisteux situé $5 \mathrm{~km}$ plus au nord. Il est en revanche possible de constater que les longues emprises linéaires étudiées ces dernières années dans le cadre du projet autoroutier au nord-ouest et au sud-est de Warcq, qui ont permis de couvrir une grande variété de positions topographiques, n'ont révélé que de rares structures anciennes, invitant à penser que ces secteurs n'avaient pas connu une occupation du sol aussi intense, notamment au cours des premiers siècles de notre ère.

Il est tentant de penser que la présence de la voie antique a conditionné l'implantation de ces nombreux sites. Or cette chaussée reste mal datée, malgré la réalisation de deux fouilles dans ce secteur, l'une au bois de Charnois, l'autre $5 \mathrm{~km}$ environ plus à l'ouest, sur la commune de Fagnon. De nombreux chercheurs attribuent ces tronçons à la voie impériale Reims-Cologne, peut-être créée au $\mathrm{I}^{\mathrm{er}}$ siècle de notre ère, malgré la largeur assez modeste des sections étudiées [14] ainsi que les nombreuses incertitudes pesant sur le parcours de cette chaussée dans la partie nord du département. Il est possible que son tracé reprenne ici un axe de circulation plus ancien, dont témoignerait la grande poutre en chêne découverte en 1989 en bordure de Meuse [15], probable élément d'une pile de pont taillé autour de 176 av. J.C. Par ailleurs, il n'est peut-être pas anodin que la Table de Peutinger indique l'existence d'un relais dénommé Mose au niveau du franchissement de ce fleuve par la voie Reims-Cologne. Faut-il le chercher sur le territoire de Warcq, par exemple au lieu-dit « Saint-Hilaire », emplacement supposé d'un habitat se développant près de la chaussée, ou sur celui de Charleville-Mézières, immédiatement à l'est?

Au-delà de ces éléments topographiques, nécessaires pour comprendre la vie d'un territoire, il paraît également intéressant de noter qu'un ensemble abondant de données a été recueilli au cours de ces fouilles, notamment sur le mode de vie des habitants de ce secteur. Il ne nous appartient pas ici de développer cet aspect, alors que plusieurs analyses sont toujours en cours. On se contentera donc de signaler par exemple le développement notable de nos connaissances dans le domaine des pratiques alimentaires [16]. Par ailleurs, parmi les nombreuses activités artisanales attestées dans ces établissements, on citera plus particulièrement la découverte d'un four de potier près de la clôture du domaine de la «RD $16 »$, celle de petites billes de «bleu égyptien » dans le bâtiment à pavillons d'angle de ce même site, mais aussi les activités métallurgiques et de meunerie détectées sur l'emprise de fouille de « La Sauce ». Les opérations organisées dans cette commune ont également permis de développer une réflexion sur les techniques de construction et les matériaux utilisés dans cette aire géographique aux cours des premiers siècles de notre ère. Les thermes fouillés dans la partie nord de Warcq ont notamment livré de nombreux éléments architectoniques, certains témoignant de sources d'approvisionnement assez éloignées, dénotant donc une certaine aisance des propriétaires des lieux. Enfin, la découverte d'une quantité importante de fragments d'ardoises et de tuiles sur chacun des établissements fouillés à Warcq renouvelle notre connaissance des modes de couverture antiques dans ce secteur.

Les données recueillies à partir de ces fouilles, qui permettent d'obtenir une première ébauche de travail, devront être complétées et améliorées grâce aux nouvelles opérations organisées dans les années à venir au sein d'une commune en pleine expansion. Une attention particulière devra par exemple être accordée aux parcelles situées immédiatement au nord et à l'est de l'emprise de fouille de «La Sauce ». Ces terrains pourraient en effet être aménagés prochainement, et
[13] Georges-Leroy et alii, p. 22.

[14] Largeur conservée à la base de $6 \mathrm{~m}$ environ à Warcq «Bois de Charnois » et de 6,5 m à Fagnon « Le Boctier» (à propos de l'intervention réalisée en 2003 à Fagnon, cf. Nicolas 2003).

[15] LEMANT et alii 2000, p. 40 et 41.

[16] De nombreuses données concernant ce sujet ont été acquises grâce aux multiples études réalisées sur ces sites, qu'elles s'intéressent aux carpo-restes (F. Toulemonde, Muséum national d'Histoire naturelle), au mobilier céramique (C. Chaidron, Arkéocéra, et A. Corsiez, Res Fabrum), aux restes fauniques (T. Oueslati, Université Lille 3, et P. Gambier, CD 08) ou encore aux meules (M. Pieters, Centre ardennais de recherche archéologique). 
donc étudiés par le biais de l'archéologie préventive, car ils se trouvent entre la ZAC actuelle, I'échangeur de la future A304 et la route départementale qui permettra de contourner Charleville-Mézières par l'ouest.

L'établissement rural identifié au bord de la plaine alluviale, au lieu-dit «La Sauce », se développe en effet certainement dans ces directions, comme la découverte de nombreux éléments de couverture en terre cuite près de l'extrémité orientale de l'emprise invite à le penser. Par ailleurs, le riche matériel métallique recueilli à quelques mètres d'un probable bâtiment d'exploitation renvoie à la sphère domestique, et indique un certain confort. La partie résidentielle du domaine reste donc à découvrir. Celle-ci pourrait être dotée d'une partie thermale si I'on en juge par la nature de certains éléments de mobilier trouvés épars sur ce chantier : fragments de briques peignées et de briques circulaires ainsi que de grandes feuilles de plomb. Ces futures opérations permettront donc probablement de mieux caractériser les vestiges préservés sur ce versant nord de la vallée et, peut-être, de mieux comprendre leur relation avec ceux mis en évidence plus au sud, le long de la route départementale. S'agit-il en effet d'un même domaine s'étendant de part et d'autre d'un cours d'eau [17] ou de deux exploitations distinctes mais synchrones [18], qui répondaient à des besoins complémentaires ? Une telle proximité géographique, entre deux groupes denses de vestiges partiellement appréhendés, reste en tout cas étonnante [19].

Parmi les autres questions restant en suspens, on évoquera le cas des thermes dégagés ces derniers mois dans la partie nord de Warcq, qui occupaient vraisemblablement l'extrémité occidentale d'une vaste aile résidentielle. Le soin accordé à sa construction et la richesse de son décor, mais aussi ses proportions, qui ne peuvent être comparées aujourd'hui - dans le département des Ardennes - qu'à celles des bains d'une villa fouillés dans les années 1970 à Carignan « Le Platé »[20], indiquent l'importance sociale des maîtres de ce lieu. Gageons que des fouilles programmées réalisées à l'est de cette partie thermale contribueraient à améliorer grandement nos connaissances de l'occupation du sol à l'époque romaine, du moins dans cette portion du territoire ardennais considérée encore il y a peu comme terra incognita.

[17] À l'image par exemple du grand domaine de Bertrange « in Bourmicht » au Luxembourg, composé de deux ensembles se développant sur les versants d'une vallée (une grande demeure isolée à l'intérieur d'une enceinte de 2,4 hectares et, de l'autre côté du ruisseau, une pars rustica s'étendant sur près de 5 hectares), non loin d'une voie antique reliant l'axe Reims-Trèves au vicus de Dalheim (FERDIÈre et alii 2010, p. 362).

[18] Une courte distance (300 m) séparait par exemple deux villae de dimensions voisines fouillées sur la commune de Roye (《Le Puits à Marne »), dans la Somme, à $700 \mathrm{~m}$ d'une voie antique (ColLART 1996, p.132-137), ou les deux établissements étudiés à Longueil-Sainte-Marie $(500 \mathrm{~m})$, dans la vallée de l'Oise (MARÉCHAL 2009, p. 208-211).

[19] Sur le plateau lorrain, entre la vallée de la Seille et la Nied Française, où un site antique est trouvé tous les 75 hectares étudiés (soit un chiffre proche de ceux obtenus plus globalement en Gaule Belgique selon l'auteur), J.-D. Laffite précise que la plupart des établissements ruraux antiques sont éloignés de plus de $500 \mathrm{~m}$ les uns des autres (LAFFITE 1999, p. 263). Plus au sud, en Nord Rhône-Alpes, des opérations de prospections et de fouilles ont mis en évidence des intervalles compris entre 300 et $700 \mathrm{~m}$ pour certains sites antiques contemporains (DE KLIJN et alii 1996, p. 282).

[20] GABER 1977.

Bernigaud, Nicolas, Ouzoulias, Pierre, Lepetz, Sébastien, Wiethold, Julian, Zech-Matterne, Véronique, SÉGUIER, Jean-Marc \& REDDÉ, Michel, 2016, « Exploitations agricoles et pratiques agro-pastorales dans les campagnes du nord-est de la Gaule (II ${ }^{\mathrm{e}}$ s. av. J.-C.-ve s. ap. J.-C.) : I'apport des données de l'archéologie préventive d'Île-de-France et de Lorraine », dans Méthodes d'analyse des différents paysages ruraux dans le nord-est de la Gaule romaine. Études comparées (hiérarchisation des exploitations ; potentialités agronomiques des sols ; systèmes de production ; systèmes sociaux), Paris, p. 63-137.

CARTron, Gael, 2010, Belval et Warcq (08), «Les Cinq Chênes » et « Gosseval », ZAC-Tranche 2, Rapport final d'opération, diagnostic archéologique, CD 08, Drac Grand Est, SRA, site de Châlons-en-Champagne.

CARTron, Gael, 2011a, «Essai sur l'occupation du sol à l'époque romaine dans la commune de Warcq (Ardennes) », Bulletin de la société archéologique champenoise 104, 2, p. 91-100.

CARTron, Gael, 2011b, Warcq (08), «A34 - Phase D», Rapport final d'opération, diagnostic archéologique, CD 08, Drac Grand Est, SRA, site de Châlons-en-Champagne. 
Cartron, Gael \& Duvauchelle, Anika, 2015, « Un aménagement en bois et une hache en fer : les vestiges mérovingiens découverts dans le fond de la vallée de la This, sur la commune de Belval (Ardennes) », Bulletin de la société archéologique champenoise 110, 2, p. 49-68.

Collart, Jean-Luc, 1996, «La naissance de la villa en Picardie : la ferme gallo-romaine précoce », dans D. Bayard \& J.-L. Collart (éd.), De la ferme indigène à la villa romaine. La romanisation des campagnes de la Gaule, Actes du 2e colloque de l'association AGER, Amiens (Somme), 23-25 sept. 1993, Revue archéologique de Picardie, numéro spécial 11, p. $121-156$. Corbiau, Marie-Hélène, 1983, «La chaussée romaine Reims-Cologne entre la Meuse et Bastogne », Archaeologia Belgica 255, Miscellanea archaeologica in honorem H. Roosens, Bruxelles, p. 145-164.

CorbiaU, Marie-Hélène \& YANTE, Jean-Marie, 2010, « Le réseau routier antique : adaptation, survie, déclassement au Moyen Âge. Exemple d'itinéraire entre Arlon et la Meuse», dans J.M. Yante \& A.M. Bultot-Verleysen (éd.), Autour du «village » : établissements humains, finages et communautés rurales entre Seine et Rhin (IVe-XIII ${ }^{e}$ siècles), Actes du colloque international de Louvain-la-Neuve, 16-17 mai 2003, Louvain-la-Neuve, p. 199-217.

De Klijn, Hans, Motte, Sylvain \& VICherd, Georges, 1996, «Éléments sur la romanisation des campagnes en Nord Rhône-Alpes », dans D. Bayard \& J.-L. Collart (éd.), De la ferme indigène à la villa romaine. La romanisation des campagnes de la Gaule, Actes du 2e colloque de I'association AGER, Amiens (Somme), 23-25 sept. 1993, Revue archéologique de Picardie, numéro spécial 11, p. 271-286.

Duvivier, M.-F.-A., 1821, Rapport sur certains lieuxdits de la commune de Warcq, qui paraissent susceptibles d'être fouillés sous le rapport des objets d'Antiquités qu'ils sont présumés renfermer, document manuscrit conservé aux archives départementales des Ardennes, cote F5.

DuvivieR, M.-F.-A., 1825, Recueil de notices historiques et archéologiques sur des objets antiques intéressans, trouvés dans diverses communes du département des ardennes, document manuscrit conservé aux archives départementales des Ardennes, cote F11.

Ferdière, Alain, Gandini, Cristina, Nouvel, Pierre \& Collart, Jean-Luc, 2010, « Les grandes villae "à pavillons multiples alignés" dans les provinces des Gaules et des Germanies : répartition, origine et fonctions », Revue archéologique de I'Est 59, 2, p. 357-446.

Frezouls, Edmond, 1977, « Informations archéologiques. Circonscription de Champagne-Ardenne », Gallia 35, 2, p. 389-418.

GABER, Stéphane, 1977, «La villa gallo-romaine de Maugré à Carignan », Revue historique ardennaise 12, p. 1-8. Georges-Leroy, Murielle, Bock, Jérôme, DAMBrine, Etienne, DuPoueY, Jean-Luc \& LAFfite, Jean-Denis, 2014, «Parcellaires et habitat antiques des forêts du plateau de Haye en Lorraine : bilan et perspectives », dans M. Reddé (éd.), Les parcellaires conservés sous forêt, Dossiers du programme européen " Rural Landscape in north-eastern Roman Gaul ", Paris, p. 1-27.

GUCKER, David, 2010, Belval et Warcq (08), «Les Cinq Chênes » et «La Haie de Gosseval », Une nécropole de la Tène A (Aisne - Marne IIB-IIC), Rapport final d'opération, diagnostic archéologique, Inrap, Drac Grand Est, SRA, site de Châlonsen-Champagne.

LAFFITE, Jean-Denis, 1999, «L'occupation du sol en milieu rural à l'époque gallo-romaine entre la Seille et la Nied Française », Les cahiers lorrains 3, p. 259-274.

LAURELut, Christophe, 2003, Charleville-Mézières (08), «Plateau de Berthaucourt », Rapport final d'opération, fouille archéologique, Inrap, Drac Grand Est, SRA, site de Châlons-en-Champagne.

LAURelut, Christophe, 2012, Charleville-Mézières (08), « Rue de Castrice » et « Rue des Noires Terres », Une agglomération secondaire méconnue : la ville antique de Castrice (Montcy-Saint-Pierre), Rapport final d'opération, suivi de travaux, Inrap, Drac Grand Est, SRA, site de Châlons-en-Champagne.

LEMANT, Jean-Pierre, 1984, «Warcq-St Hilaire : le passage de la voie romaine Reims-Cologne au site de Saint-Hilaire à Warcq », Les Amis du Vieux Warcq 8, p. 9-19.

Lemant, Jean-Pierre, Tegel, Willy \& VAnmoerkerke, Jan, 2000, « Warcq, "Pont" », Bulletin scientifique régional 1997, Drac Grand Est, SRA, site de Châlons-en-Champagne.

MARÉCHAL, Denis, 2009, «Le village de Longueil-Sainte-Marie et les autres formes d'implantation agricole dans la moyenne vallée de l'Oise », Aquitania, supplément 17, Colloque Ager VIII, Toulouse, 2007, Bordeaux, p. 199-215.

Nrcolas, David, 2003, Fagnon (08), « Le Boctier » et « Roux Sainte-Fosse », Rapport final d'opération, sondages archéologiques, Drac Grand Est, SRA, site de Châlons-en-Champagne.

Nrcolas, David, 2011, Les Ardennes (Carte archéologique de la Gaule, 08), Paris.

RoseAU, Bertrand, à paraître, Warcq (08), «La Sauce - tombe à char », Rapport final d'opération, fouille archéologique, CD 08-Inrap.

WATerlot, Gérard, Bonte, Antoine, Destombes, Jean-Paul \& Lemoine, Marcel, 1960, Notice de la carte géologique de la France à $1 / 50000$ - Renwez - XXIX-9 - BRGM. 